\title{
Diastereoselective Alkylation of $\beta$-Amino Esters: Structural and Rate Studies Reveal Alkylations of Hexameric Lithium Enolates
}

\author{
Anne J. McNeil,${ }^{\dagger}$ Gilman E. S. Toombes, ${ }^{\ddagger}$ Sol M. Gruner, ${ }^{\ddagger}$ Emil Lobkovsky, ${ }^{\dagger}$ David B. \\ Collum, ${ }^{* \dagger}$ Sithamalli V. Chandramouli, ${ }^{\S}$ Benoit J. Vanasse, ${ }^{\S}$ and Timothy A. Ayers ${ }^{\S}$ \\ Contribution from the ${ }^{\dagger}$ Department of Chemistry and Chemical Biology, Baker Laboratory, Cornell \\ University, Ithaca, New York 14853-1301, ${ }^{\ddagger}$ Physics Department, Clark Hall, Cornell University, \\ Ithaca, New York 14853-2501, and ${ }^{\S}$ Aventis, Process Development Chemistry, Bridgewater, New \\ Jersey 08807
}

Structure Chart

\section{Spectroscopic Data for Characterization}

1. $\quad{ }^{1} \mathrm{H}$ and ${ }^{13} \mathrm{C}$ NMR spectra of 4 . S6

2. $\quad{ }^{1} \mathrm{H}$ and ${ }^{13} \mathrm{C}$ NMR spectra of $\mathbf{5}$. S7

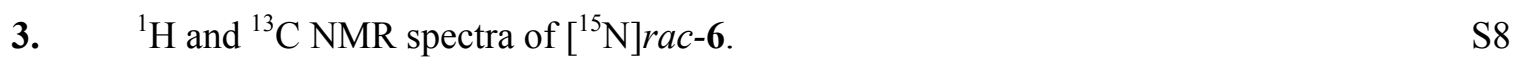

4. $\quad{ }^{1} \mathrm{H}$ and ${ }^{13} \mathrm{C}$ NMR spectra of $\left[{ }^{15} \mathrm{~N}\right](S)-6 . \quad$ S9

5. $\quad{ }^{1} \mathrm{H}$ and ${ }^{13} \mathrm{C}$ NMR spectra of $7 . \quad \mathrm{S} 10$

6. $\quad{ }^{1} \mathrm{H}$ and ${ }^{13} \mathrm{C}$ NMR spectra of a mixture of 7:8 (1:4). S11

7. ${ }^{1} \mathrm{H}$ and ${ }^{13} \mathrm{C}$ NMR spectra of $\mathbf{1 2}$ S12

8. $\quad{ }^{1} \mathrm{H}$ and ${ }^{13} \mathrm{C}$ NMR spectra of $\mathbf{1 4}$ S13

9. $\quad{ }^{1} \mathrm{H}$ and ${ }^{13} \mathrm{C}$ NMR spectra of a mixture of $\mathbf{1 4 : 1 5} \quad \mathrm{S} 14$

\section{Control Experiments}

10. LiHMDS-mediated enolization of tosylate salt 2. S15

11. LiHMDS-mediated enolization and cyclization of 4 S15

12. LiHMDS-mediated enolization of 5 S15

13. LiHMDS-mediated enolization of free base $6 . \quad S 16$

14. LiHMDS-mediated enolization and cyclization of 7/8. S16

15. In-situ formation of lactam $\mathbf{1 4}$ with LiHMDS/rac-9.

16. Formation of stilbene $\mathbf{1 3}$ from LiHMDS/3. $\quad$ S18 
17. GC analysis of LiHMDS with BnBr. $\quad$ S18

18. Table of $k_{\text {rel }}$ for alkylations. $\quad \mathrm{S} 18$

19. Plot of $k_{\text {obsd }}$ versus [28] for the alkylation of 9 with BnBr. S19

20. Table of data for plot in Figure $19 . \quad$ S19

21. Plot of $k_{\text {obsd }}$ versus [LiHMDS] for the alkylation of 9 with $\mathrm{BnBr}$.

22. Table of data for plot in Figure 21.

23. Plot of $k_{\text {obsd }}$ versus [ $\left.\mathrm{LiBr}\right]$ for the alkylation of 9 with $\mathrm{BnBr}$. $\quad$ S21

24. Table of data for plot in Figure 23. $\quad$ S21

\section{Single-Crystal X-ray Structure Data}

25. Crystal structure: ORTEP and experimental details.

\section{Powder Diffraction Data}

26. Experimental protocol and results. $\quad$ S23

27. Plot of the raw data versus the calculated fit. $\quad$ S23

\section{Small-Angle X-Ray Scattering Data}

$\begin{array}{lll}\text { 28. } & \text { Experimental details. } & \text { S24 }\end{array}$

29. Plot of the scattering intensity as a function of momentum transfer. $\quad$ S24

30. Plot of the additional scattering as a function of momentum transfer.

\section{Solubility Studies Using In-situ IR}

31. Calibration plot.

$\begin{array}{ll}\text { 32. } & \text { Supersaturation. }\end{array}$

33. Variable-temperature studies. $\quad$ S28

$\begin{array}{lr}\text { 34. Isolation of solid } r a c-9 . & \text { S28 }\end{array}$

${ }^{6}$ Li NMR Spectroscopic Studies

35. $\quad{ }^{6} \mathrm{Li} \mathrm{NMR}$ spectra recorded on $\left[{ }^{6} \mathrm{Li},{ }^{15} \mathrm{~N}\right](S)-9$ and $\left[{ }^{6} \mathrm{Li},{ }^{15} \mathrm{~N}\right] \mathrm{rac}-\mathbf{9}$. 
36. $\quad{ }^{6} \mathrm{Li} \mathrm{NMR}$ spectra recorded on $\left[{ }^{6} \mathrm{Li}\right](R)-9$ at various temperatures.

37. $\quad{ }^{6} \mathrm{Li} \mathrm{NMR}$ spectra recorded on $\left[{ }^{6} \mathrm{Li}\right] \mathrm{rac}-9$ at various temperatures.

38. $\quad{ }^{6} \mathrm{Li}$ NMR spectra recorded on a mixture of $\left[{ }^{6} \mathrm{Li}\right](R)-9$ and $\left[{ }^{6} \mathrm{Li}\right] \mathrm{rac}-9$ at various temperatures.

39. $\quad{ }^{6} \mathrm{Li} \mathrm{NMR}$ spectra recorded on a mixture of $\left[{ }^{6} \mathrm{Li}\right](R)-9$ and $\left[{ }^{6} \mathrm{Li}\right] \mathrm{rac}-9$ at various [enolate].

40. Plot of the mole fraction of the aggregate versus [enolate].

41. Table of data for the plot in Figure 40.

42. $\quad{ }^{6} \mathrm{Li}$ NMR spectra recorded on a mixture of $\left[{ }^{6} \mathrm{Li}\right](R)-9$ and $\left[{ }^{6} \mathrm{Li}\right] \mathrm{rac}-\mathbf{9}$ in various [THF].

43. Plot of the mole fraction of the aggregate versus [THF]. S36

44. Table of data for the plot in Figure 43. S36

45. $\quad{ }^{6} \mathrm{Li}$ NMR spectra recorded on mixtures of $\left[{ }^{6} \mathrm{Li}\right](\mathrm{S})-9$ and $\left[{ }^{6} \mathrm{Li}\right] \mathrm{rac}-9$ at $-50{ }^{\circ} \mathrm{C} . \quad \mathrm{S} 37$

46. $\quad{ }^{6} \mathrm{Li}$ NMR spectra recorded on mixtures of $\left[{ }^{6} \mathrm{Li}\right](R)-9$ and $\left[{ }^{6} \mathrm{Li}\right] \mathrm{rac}-9$ at $-50{ }^{\circ} \mathrm{C} . \quad \mathrm{S} 38$

47. Plot of the mole fraction of the aggregate versus the mole fraction of $R$. S39

48. Table of data for the plot in Figure $47 . \quad$ S39

49. $\quad{ }^{6} \mathrm{Li}$ NMR spectra recorded on $\left[{ }^{6} \mathrm{Li}\right](R)-9$ and $\left[{ }^{6} \mathrm{Li},{ }^{15} \mathrm{~N}\right] \mathrm{LiHMDS}$ at various [THF]. $\quad \mathrm{S} 40$

50. $\quad{ }^{6} \mathrm{Li}$ NMR spectra recorded on $\left[{ }^{6} \mathrm{Li}\right] \mathrm{rac}-9$ and $\left[{ }^{6} \mathrm{Li},{ }^{15} \mathrm{~N}\right] \mathrm{LiHMDS}$ at various [THF]. S41

51. ${ }^{6} \mathrm{Li} \mathrm{NMR}$ spectra recorded on mixtures of $\left[{ }^{6} \mathrm{Li}\right](R)-9$ and $\left[{ }^{6} \mathrm{Li}\right] \mathrm{LiBr} . \quad \mathrm{S} 42$

52. $\quad{ }^{6} \mathrm{Li}$ NMR spectra recorded on mixtures of $\left[{ }^{6} \mathrm{Li}\right](R)-9$ and $\left[{ }^{6} \mathrm{Li}\right] \beta \mathrm{HV}$ al. $\quad \mathrm{S} 43$

\section{MNDO Computations}

53. Relative heats of formation. $\quad$ S44

54. Effect of symmetry on heats of formation for hexamers. S45

55. Thermoneutral opening of the $\mathrm{R}_{3} \mathrm{~S}_{3}$ hexamer to the ladder. $\mathrm{S} 45$

56. Transition structures for anti alkylation of $\mathrm{R}_{3} \mathrm{~S}_{3}$ ladder. $\quad \mathrm{S} 46$

57. Transition structures for anti alkylation via the monomer. $\quad$ S47

58. Transition structures for anti alkylation via the $\mathrm{R}_{1} \mathrm{~S}_{1}$ doubly-chelated dimer. $\quad \mathrm{S} 48$

\section{Mathematical Derivations}

59. Introduction to the hexamer fitting protocol. S49

60. Boltzmann distribution. S51 
61. Multiplicity. S53

62. Chemical potential. S54

63. The statistical case.

$\begin{array}{ll}\text { 64. The parametric method. } & \text { S57 }\end{array}$

65. Fitting the experimental data with the parametric method. S60

66. Equilibrium constants. S61

67. Heptamer fit to data. $\quad$ S63

68. Generic tetramer Job plot.

69. Dimer-tetramer Job plot. S66 


\section{Structure Chart}<smiles>COC(=O)C[C@H](C)[NH2+][O-]</smiles>

2<smiles>COC(=O)C[C@H](C)N</smiles>

(R)-6<smiles>COC(=O)[C@H](Cc1ccccc1)[C@H](C)N</smiles><smiles>COC(=O)[C@H](CBr)[C@H](C)NC[Ga]</smiles>

11

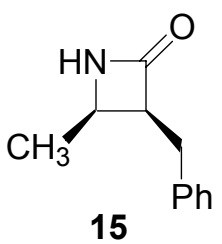<smiles>N#Cc1cccc(CBr)c1</smiles>

3

$\left(\mathrm{ArCH}_{2} \mathrm{Br}\right)$<smiles>COC(=O)C[C@H](C)N</smiles>

(S)-6<smiles>CO/C(Cl)=C/[C@H](C)N</smiles><smiles>C[C@H]1NC(=O)[C@H]1CBr</smiles>

12

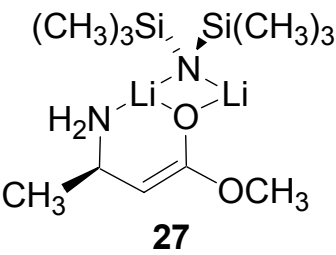<smiles>COC(=O)[C@H](C[Ge])[C@H](C)N</smiles><smiles>COC(=O)[C@H](C[Se])[C@@H](C)N</smiles><smiles>COC(=O)C[C@H](C)N</smiles>

rac-6<smiles>[3H][P+](C[C@@H](C(=O)OC)[C@H](C)N)c1ccccc1</smiles><smiles>CO/C(Cl)=C/[C@H](C)N</smiles><smiles>Br/C=C/[Bi]</smiles>

13

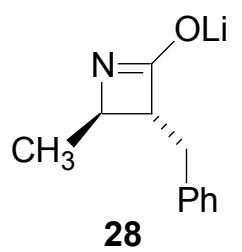

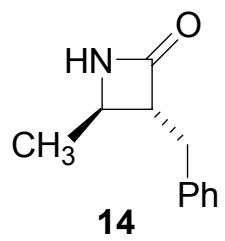<smiles>COC(=O)C(C[Te])(C[Te])[C@@H](C)N</smiles><smiles>CC1N=C(O)C1Cc1ccccc1</smiles> 


\section{Spectroscopic Data for Characterization}
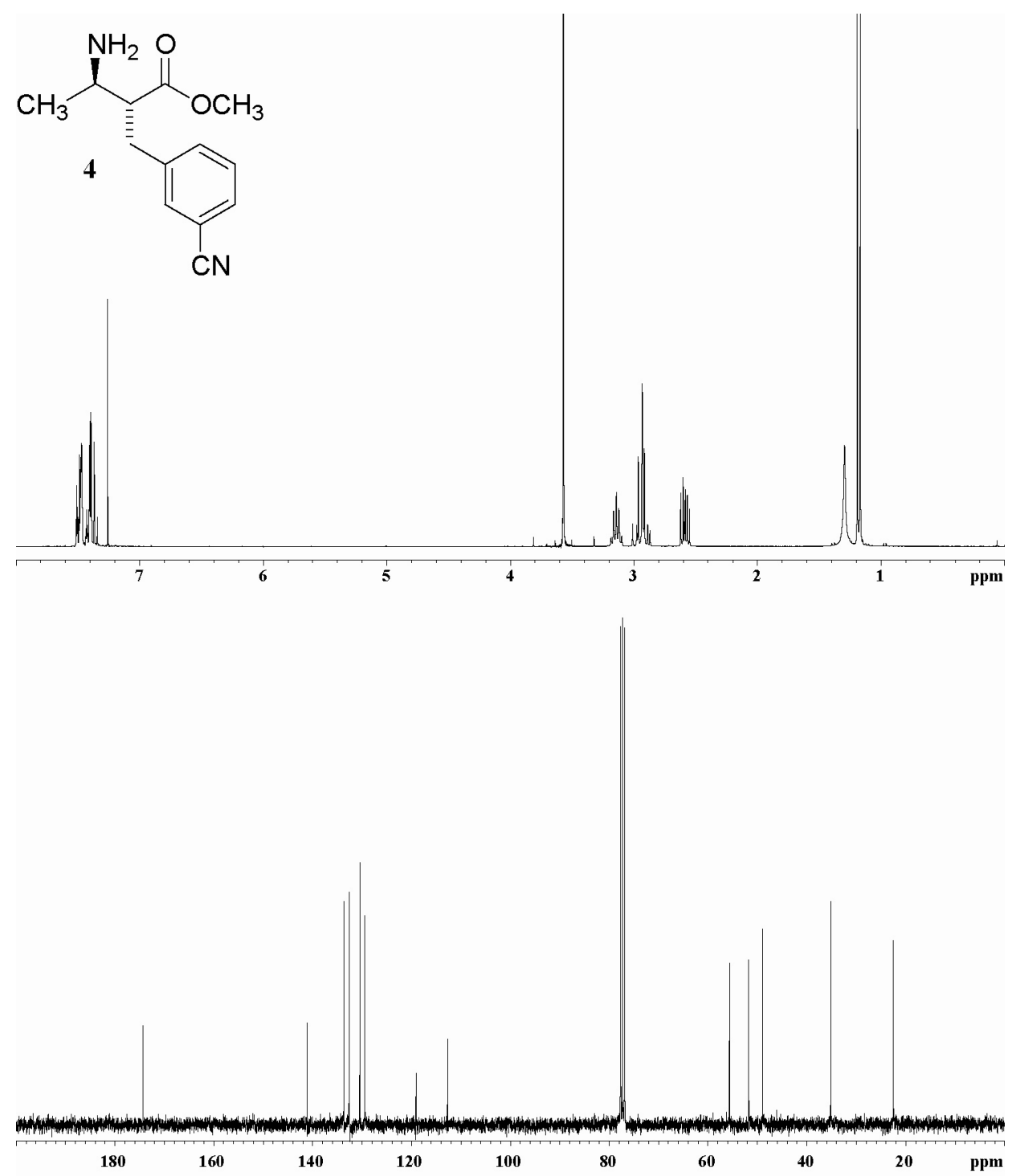

1. ${ }^{1} \mathrm{H}$ NMR (300 MHz, $\left.\mathrm{CDCl}_{3}\right): \delta 1.17(\mathrm{~d}, J=6.5 \mathrm{~Hz}, 3 \mathrm{H}), 1.27$ (bs, 2H), 2.54-2.62 (m, 1H), $2.89(\mathrm{dd}, J=5.7,13.5 \mathrm{~Hz}, 1 \mathrm{H}), 2.97(\mathrm{dd}, J=10,13.2 \mathrm{~Hz}, 1 \mathrm{H}), 3.14$ (pentet, $J=6.3 \mathrm{~Hz}, 1 \mathrm{H}), 3.56$ (s, 3H), 7.26-7.48 (m, 4H). ${ }^{13} \mathrm{C}$ NMR (75 MHz, $\left.\mathrm{CDCl}_{3}\right): \delta 22.5,35.1,48.9$, 51.8, 55.6, 112.7, $119.1,129.5,130.5,132.6,133.6,141.1,174.3$. 

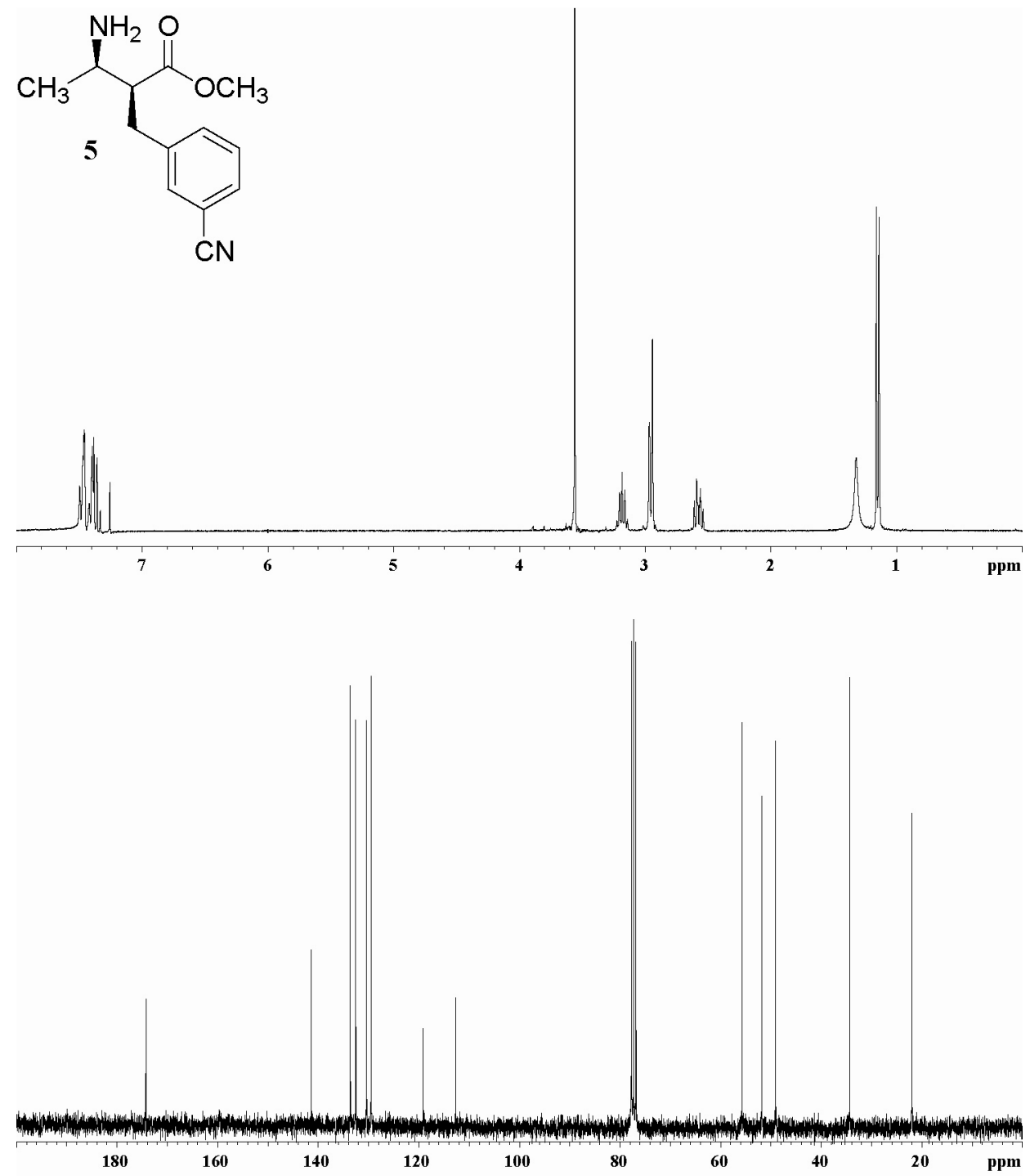

2. ${ }^{1} \mathrm{H}$ NMR (300 MHz, $\left.\mathrm{CDCl}_{3}\right): \delta 1.15(\mathrm{~d}, J=6.6 \mathrm{~Hz}, 3 \mathrm{H}), 1.32(\mathrm{bs}, 2 \mathrm{H}), 2.54-2.61(\mathrm{~m}, 1 \mathrm{H})$, 2.92-3.02 (m, 1H), 3.18 (pentet, $J=6.3 \mathrm{~Hz}, 1 \mathrm{H}), 3.56(\mathrm{~s}, 3 \mathrm{H}), 7.26-7.50(\mathrm{~m}, 4 \mathrm{H}) .{ }^{13} \mathrm{C}$ NMR $(75$ $\left.\mathrm{MHz}, \mathrm{CDCl}_{3}\right): \delta 21.9,34.3,49.0,51.8,55.7,112.7,119.1,129.5,130.4,132.5,133.6,141.4$, 174.3 . 

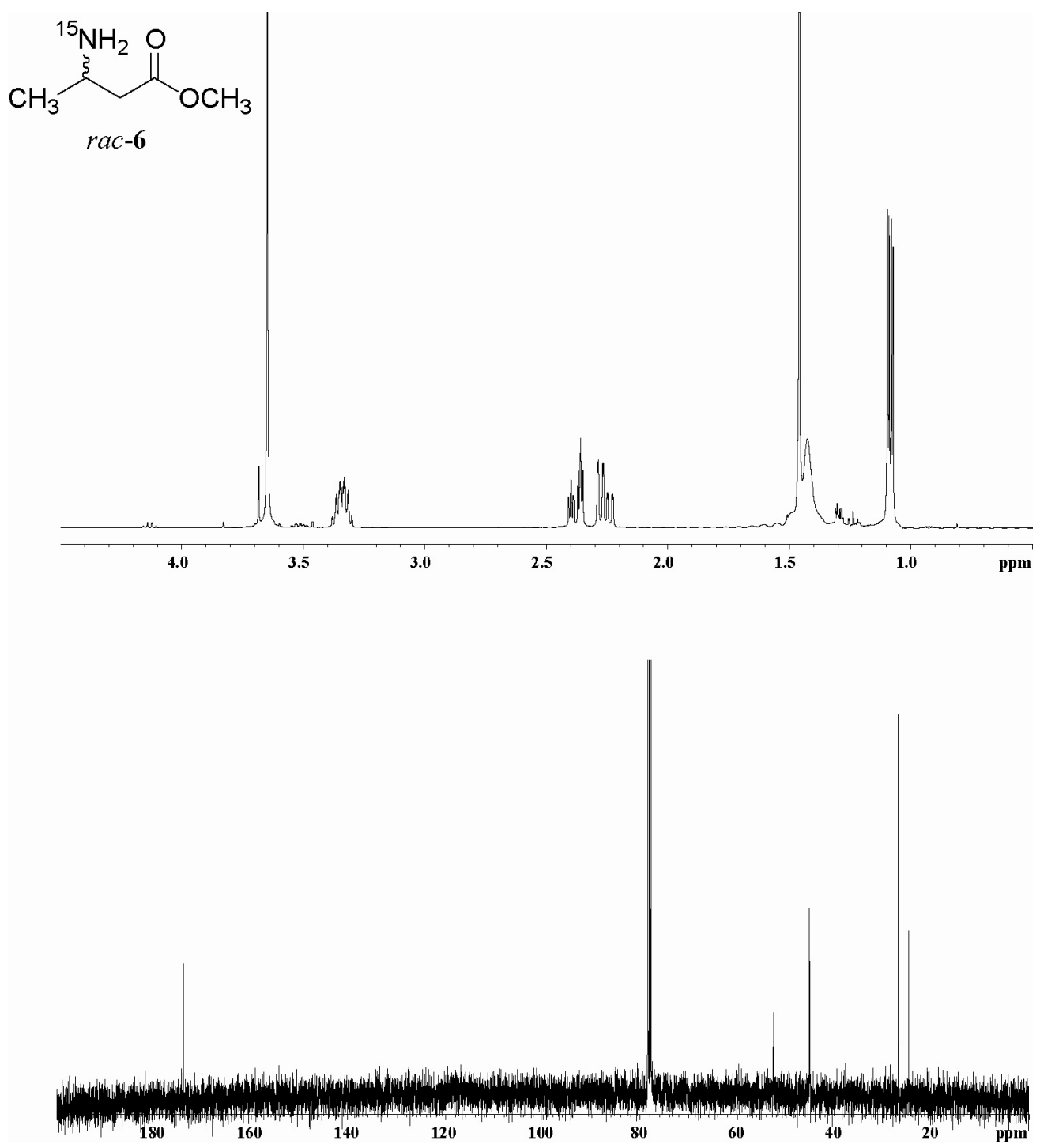

3. ${ }^{1} \mathrm{H}$ NMR (400 MHz, $\left.\mathrm{CDCl}_{3}\right): \delta 1.08(\mathrm{dd}, J=2.8,6.6 \mathrm{~Hz}, 3 \mathrm{H}), 1.43(\mathrm{bs}, 2 \mathrm{H}), 1.46\left(\mathrm{C}_{5} \mathrm{H}_{10}\right)$, $2.28(\mathrm{dd}, J=8,16 \mathrm{~Hz}, 1 \mathrm{H}) 2.38(\mathrm{~m}, 1 \mathrm{H}), 3.3-3.4(\mathrm{~m}, 1 \mathrm{H}), 3.65(\mathrm{~s}, 3 \mathrm{H}) .{ }^{13} \mathrm{C} \mathrm{NMR}(100 \mathrm{MHz}$, $\left.\mathrm{CDCl}_{3}\right): \delta 24.7,26.9\left(\mathrm{C}_{5} \mathrm{H}_{10}\right), 45.08,45.14,52.6,173.9$. (Note: $\mathrm{C}_{5} \mathrm{H}_{10}$ is the solvent cyclopentane.)

${ }^{15} \mathrm{~N}$-labeled $\beta$-amino esters were synthesized from $\left[{ }^{15} \mathrm{~N}\right]$ alanine via the Arndt-Eistert protocol. (Podlech, J.; Seebach, D. Liebigs Ann. 1995, 1217-1228.) 

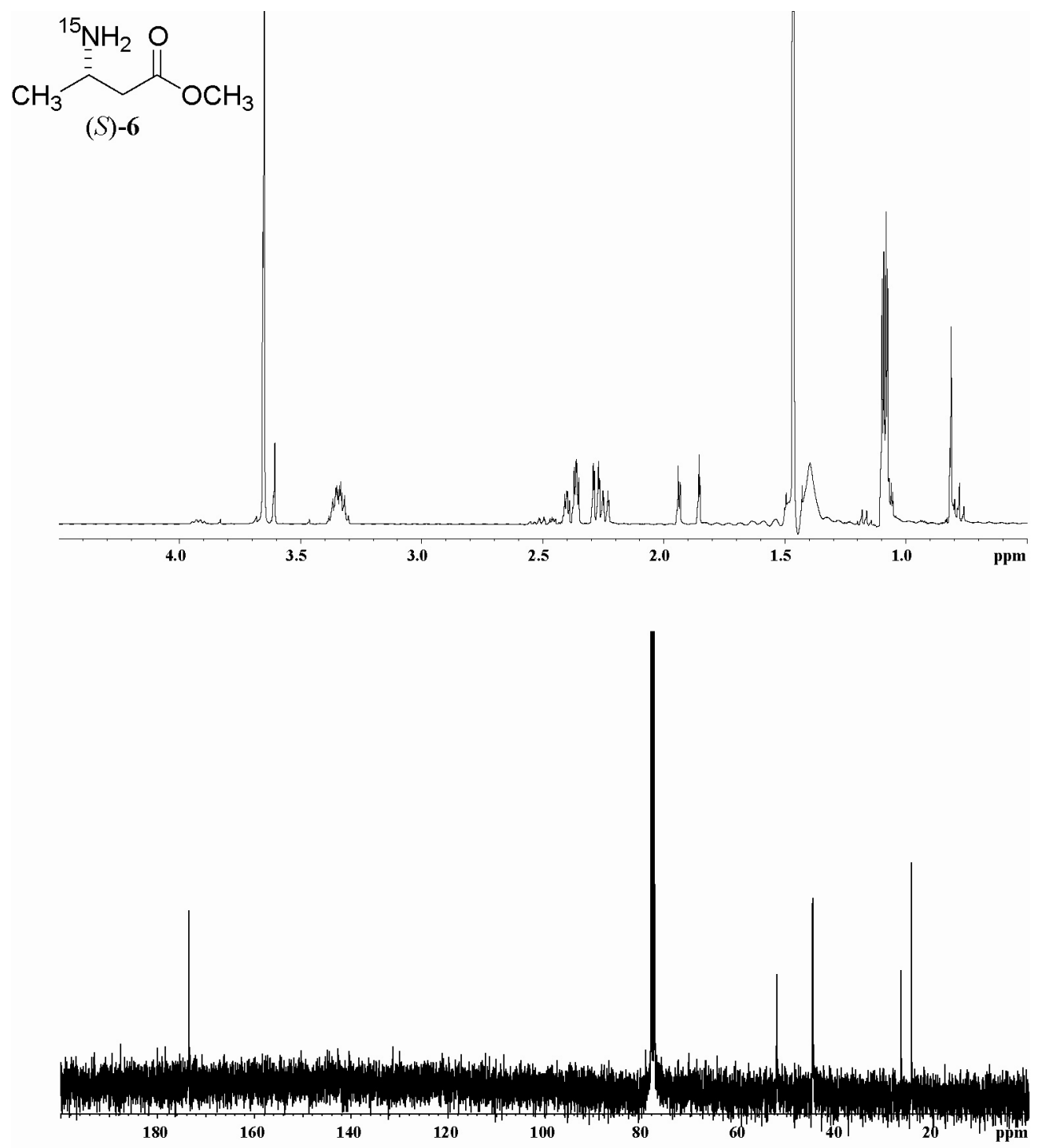

4. ${ }^{1} \mathrm{H}$ NMR (400 MHz, $\left.\mathrm{CDCl}_{3}\right): \delta 1.09$ (dd, $\left.J=2.4,6.8 \mathrm{~Hz}, 3 \mathrm{H}\right), 1.40(\mathrm{bs}, 2 \mathrm{H}), 1.46\left(\mathrm{C}_{5} \mathrm{H}_{10}\right)$, 2.26 (ddd, $J=1.5,8.8,15.6 \mathrm{~Hz}, 1 \mathrm{H}), 2.38$ (ddd, $J=3.4,4.4,15.6 \mathrm{~Hz}, 1 \mathrm{H}), 3.3-3.4(\mathrm{~m}, 1 \mathrm{H}), 3.65$ (s, 3H). ${ }^{13} \mathrm{C} \mathrm{NMR}\left(100 \mathrm{MHz}, \mathrm{CDCl}_{3}\right): \delta 24.2,26.4\left(\mathrm{C}_{5} \mathrm{H}_{10}\right), 44.6,44.7,52.1,173.5$. (Note: $\mathrm{C}_{5} \mathrm{H}_{10}$ is the solvent cyclopentane.)

${ }^{15} \mathrm{~N}$-labeled $\beta$-amino esters were synthesized from $\left[{ }^{15} \mathrm{~N}\right]$ alanine via the Arndt-Eistert protocol. (Podlech, J.; Seebach, D. Liebigs Ann. 1995, 1217-1228.) 

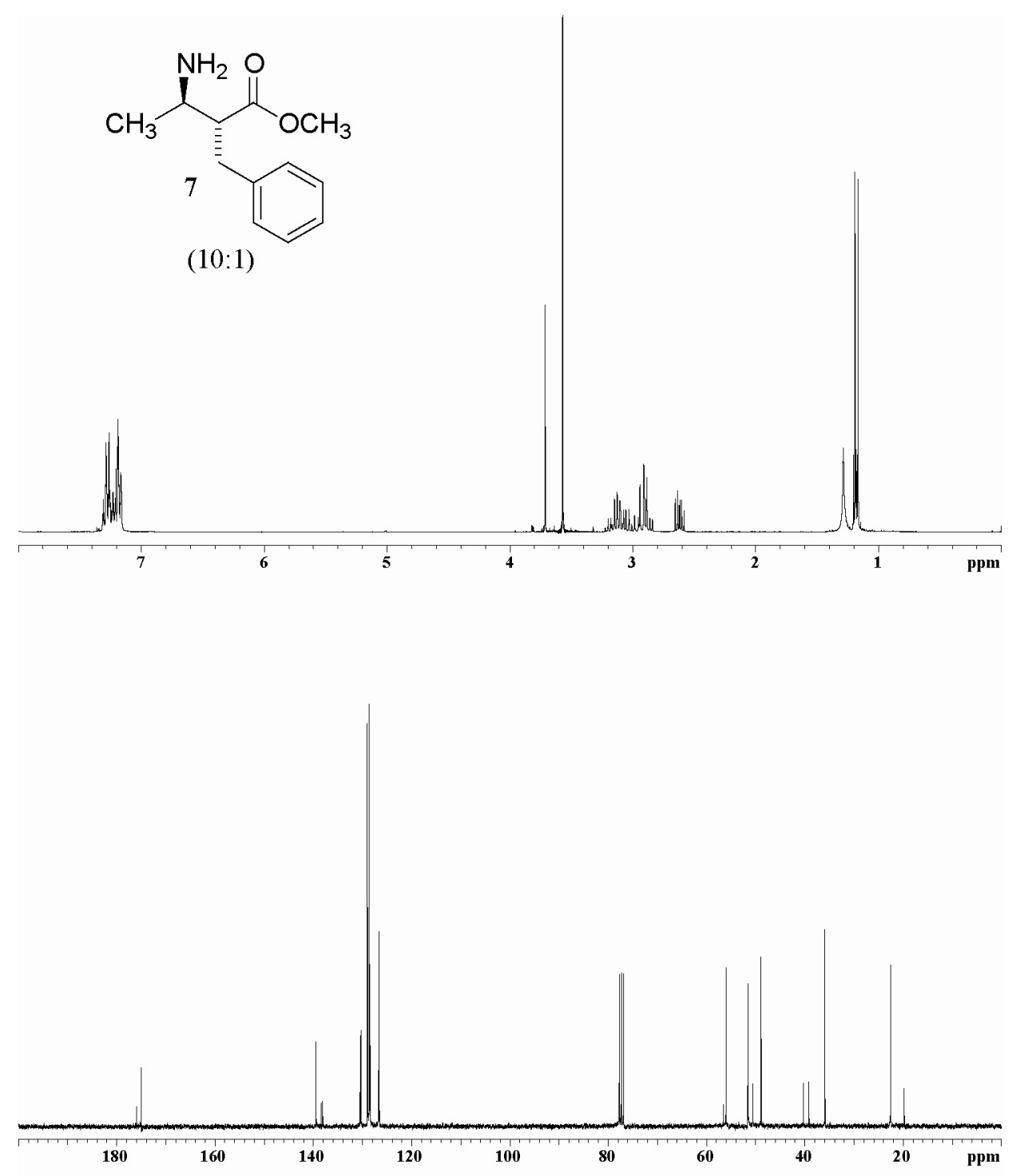

5. ${ }^{1} \mathrm{H} \mathrm{NMR}\left(300 \mathrm{MHz}, \mathrm{CDCl}_{3}\right): \delta 1.18(\mathrm{~d}, J=6.6 \mathrm{~Hz}, 3 \mathrm{H}), 1.29(\mathrm{bs}, 2 \mathrm{H}), 2.62(\mathrm{dt}, J=5.7,10.2$ $\mathrm{Hz}, 1 \mathrm{H}), 2.87$ (dd, $J=5.7,13.5 \mathrm{~Hz}, 1 \mathrm{H}), 2.94(\mathrm{dd}, J=9.3,13.2 \mathrm{~Hz}, 1 \mathrm{H}), 3.13(\mathrm{~m}, 1 \mathrm{H}), 3.57$ (s, $3 \mathrm{H}), 7.13-7.26(\mathrm{~m}, 5 \mathrm{H}) .{ }^{13} \mathrm{C} \mathrm{NMR}\left(75 \mathrm{MHz}, \mathrm{CDCl}_{3}\right): \delta 22.5,35.8,48.9,51.6,56.0,126.7,128.9$, $130.5,139.4,174.9$. 

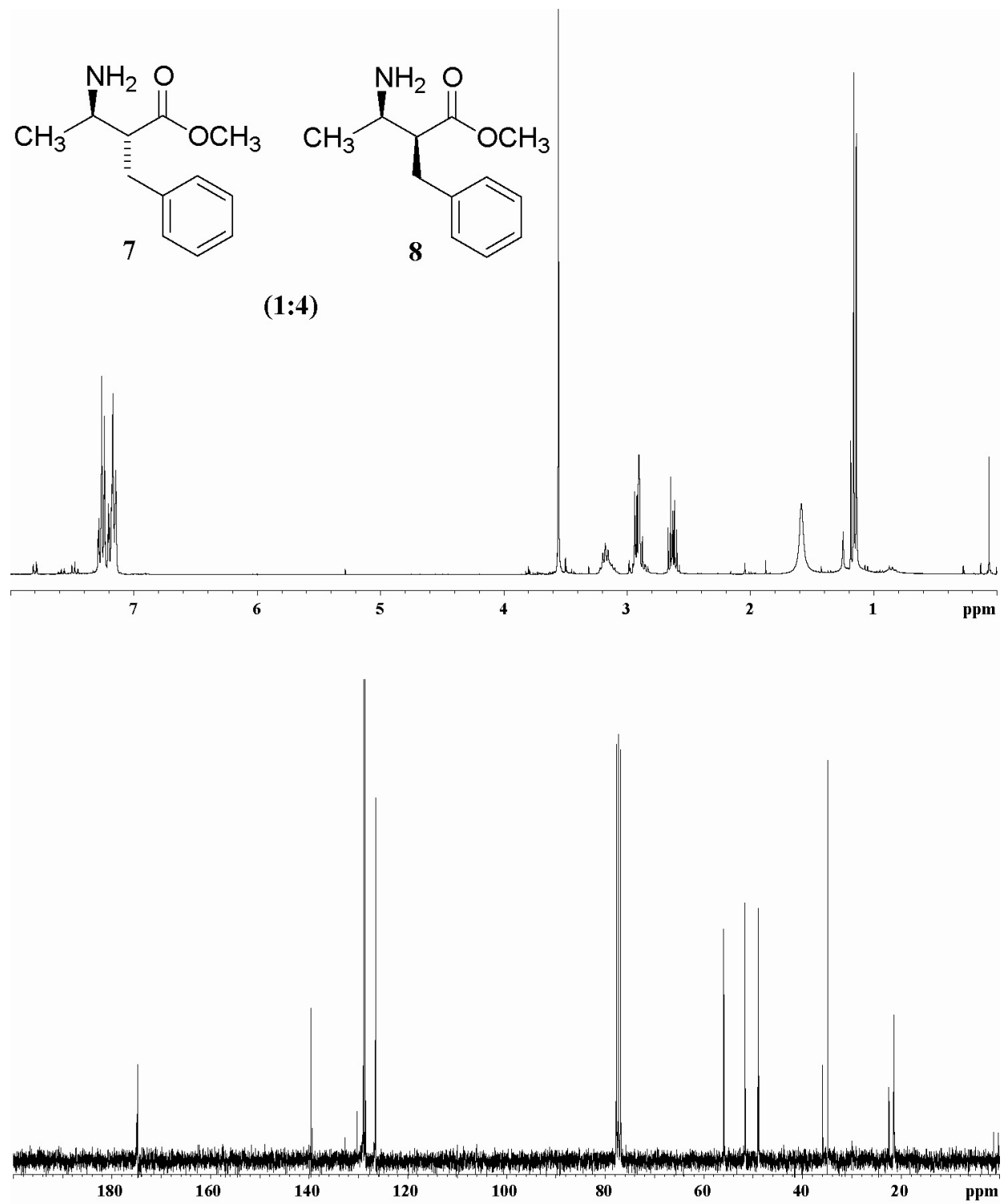

6. 8: ${ }^{1} \mathrm{H}$ NMR (300 MHz, $\left.\mathrm{CDCl}_{3}\right): \delta 1.15(\mathrm{~d}, J=6.6 \mathrm{~Hz}, 3 \mathrm{H}), 1.59$ (bs, $\left.2 \mathrm{H}\right), 2.61-2.67(\mathrm{~m}, 1 \mathrm{H})$, 2.83-2.98 (m, 2H), 3.10-3.22 (m, 1H), 3.56 (s, 3H), 7.14-7.29 (m, 5H). ${ }^{13} \mathrm{C}$ NMR (75 MHz, $\left.\mathrm{CDCl}_{3}\right): \delta 21.5,34.9,49.0,51.6,55.9,126.5,128.6,128.9,139.6,174.8$. For 7 , see Figure $5(\mathrm{p}$ S10). 

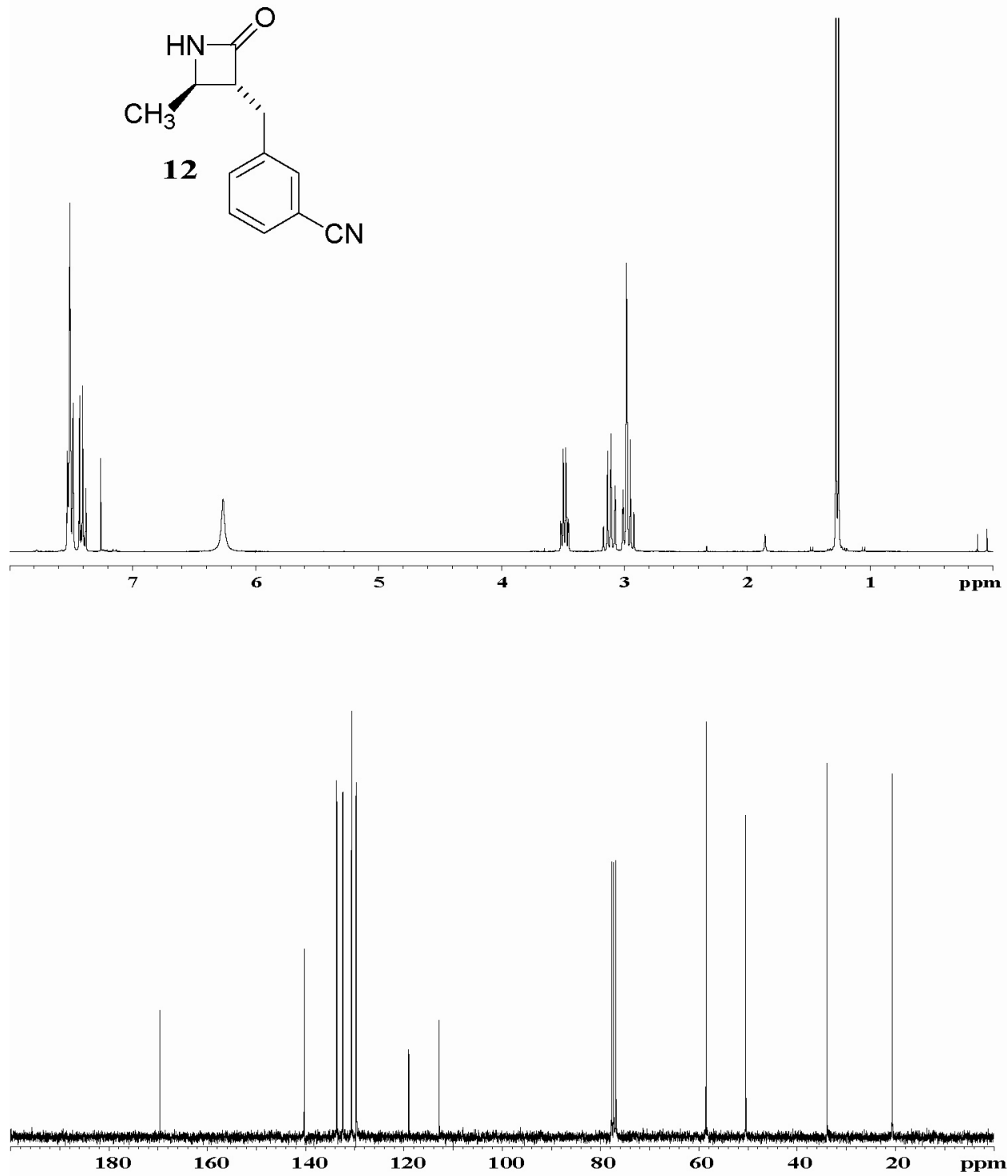

7. ${ }^{1} \mathrm{H}$ NMR (300 MHz, $\left.\mathrm{CDCl}_{3}\right): \delta 1.26(\mathrm{~d}, J=6 \mathrm{~Hz}, 3 \mathrm{H}), 2.98(\mathrm{~m}, 2 \mathrm{H}), 3.12(\mathrm{~m}, 1 \mathrm{H}), 3.48(\mathrm{dq}, J$ $=1.8,6 \mathrm{~Hz}, 1 \mathrm{H}), 6.26(\mathrm{bs}, 1 \mathrm{H}), 7.38-7.54(\mathrm{~m}, 4 \mathrm{H}) .{ }^{13} \mathrm{C} \mathrm{NMR}\left(75 \mathrm{MHz}, \mathrm{CDCl}_{3}\right): \delta 20.7,34.0$, $50.5,58.5,112.8,119.0,129.7,130.7,132.4,133.6,140.2,169.6$. 

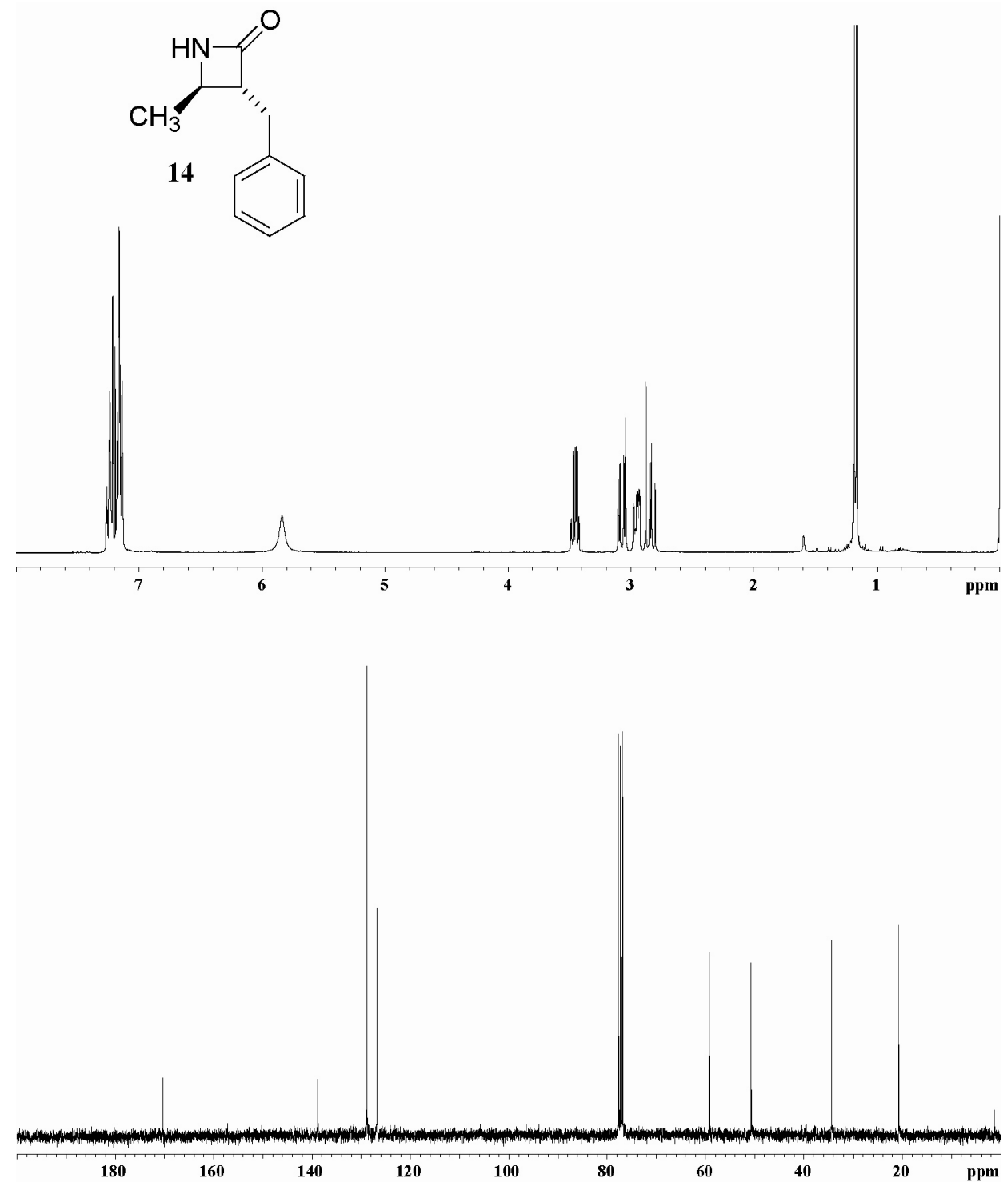

8. ${ }^{1} \mathrm{H}$ NMR (300 MHz, $\left.\mathrm{CDCl}_{3}\right): \delta 1.17(\mathrm{~d}, J=6 \mathrm{~Hz}, 3 \mathrm{H}), 2.84(\mathrm{dd}, J=9.3,13.5 \mathrm{~Hz}, 1 \mathrm{H}), 2.95$ (m, 1H), 3.08 (dd, $J=4.8,13.5 \mathrm{~Hz}, 1 \mathrm{H}), 3.45$ (dq, $J=2.1,6.3 \mathrm{~Hz}, 1 \mathrm{H}), 5.84$ (bs, 1H), 7.19-7.27 $(\mathrm{m}, 5 \mathrm{H}) .{ }^{13} \mathrm{C}$ NMR $\left(75 \mathrm{MHz}, \mathrm{CDCl}_{3}\right): \delta 20.7,34.4,50.7,59.2,126.7,128.8,128.9,138.8,170.4$. 


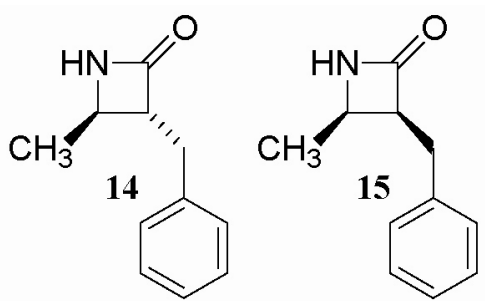

(5:1)
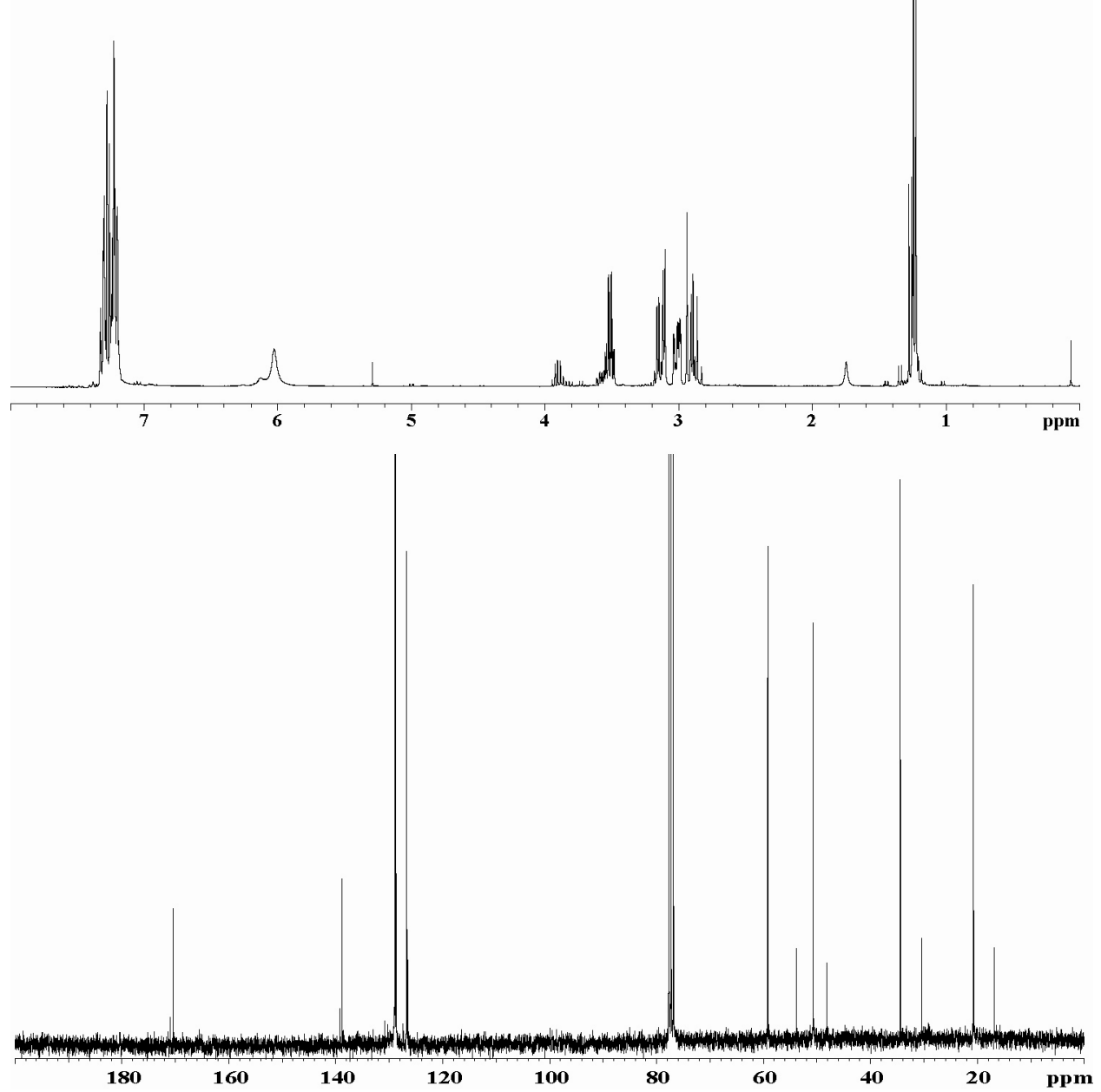

9. 15: ${ }^{1} \mathrm{H}$ NMR (300 MHz, $\left.\mathrm{CDCl}_{3}\right): \delta 1.27(\mathrm{~d}, J=6 \mathrm{~Hz}, 3 \mathrm{H}), 2.83-2.94(\mathrm{~m}, 1 \mathrm{H}), 3.00-3.04(\mathrm{~m}$, 1H), 3.10-3.20 (m, 1H), 3.55-3.61 (m, 1H), 6.13 (bs, 1H), 7.18-7.33 (m, 5H). ${ }^{13} \mathrm{C}$ NMR (75 $\mathrm{MHz}, \mathrm{CDCl}_{3}$ ): $\delta 16.9,30.4,48.1,53.8,126.6,128.6,139.2 ., 170.9$. For 14, see Figure 8 (pg S13). 


\section{Control Experiments}

\section{LiHMDS-mediated enolization of tosylate salt 2}

LiHMDS (0.167 g, $1.0 \mathrm{mmol})$ was weighed into a $15 \mathrm{~mL}$ flask and dissolved in $6.3 \mathrm{~mL}$ THF and $2.0 \mathrm{~mL}$ toluene. The solution was placed in a ReactIR vessel and cooled to $0{ }^{\circ} \mathrm{C}$ for $20 \mathrm{~min}$. LiHMDS (0.167 g, $1.0 \mathrm{mmol})$ was weighed into a $5 \mathrm{~mL}$ Kimble vial and dissolved in $1.0 \mathrm{~mL}$ THF. Tosylate salt $2(0.289 \mathrm{~g}, 1.0 \mathrm{mmol})$ was added to the $5 \mathrm{~mL}$ vial with LiHMDS to afford the free base. The mixture was initially heterogeneous (salt $\mathbf{2}$ is insoluble in THF). After a few seconds, the solution became homogeneous and then immediately a white precipitate formed (LiOTs). This heterogeneous mixture was then injected into the IR vessel containing $1.0 \mathrm{mmol}$ of LiHMDS and the spectrum was recorded within $20 \mathrm{~s}$. The absence of a $\mathrm{C}=\mathrm{O}$ absorbance indicates complete and instantaneous enolization. The enolate $\mathrm{C}=\mathrm{C}$ absorbance was at $1620 \mathrm{~cm}^{-1}$.

\section{LiHMDS-mediated enolization and cyclization of 4.}

LiHMDS (0.167 g, $1.0 \mathrm{mmol})$ was dissolved in $7.3 \mathrm{~mL}$ THF and $2.5 \mathrm{~mL}$ toluene. The solution was placed in a ReactIR vessel and cooled to $-50{ }^{\circ} \mathrm{C}$ for $20 \mathrm{~min}$. In a separate $5 \mathrm{~mL}$ Kimble vial, $4(0.266 \mathrm{~g}, 1.1 \mathrm{mmol})$ was dissolved in $0.87 \mathrm{~mL}$ THF and $100 \mu \mathrm{L}$ was injected into the IR vessel. The spectra were collected every $30 \mathrm{~s}$. The $\mathrm{C}=\mathrm{O}$ absorbance $\left(1732 \mathrm{~cm}^{-1}\right)$ decreased to the baseline with the appearance of a new absorbance at $1620 \mathrm{~cm}^{-1}$. The pseudo-first-order rate constant was $2.3 \pm 0.1 \times 10^{-3} \mathrm{~s}^{-1}$. An additional $400 \mu \mathrm{L}$ of the solution of ester 4 was injected and the reaction was quenched with $\mathrm{H}_{2} \mathrm{O}$ when the $\mathrm{C}=\mathrm{O}$ absorbance had disappeared.

Chromatographic separation of the products $\left(95 / 5 \mathrm{CH}_{2} \mathrm{Cl}_{2} / \mathrm{CH}_{3} \mathrm{OH}\right)$ provided lactam 14 in $54 \%$ yield and esters 4 and 5 in a 19 and 19\% yield, respectively.

\section{LiHMDS-mediated enolization of 5.}

LiHMDS (0.167 g, $1.0 \mathrm{mmol})$ was dissolved in $7.3 \mathrm{~mL}$ THF and $2.5 \mathrm{~mL}$ toluene. The solution was placed in a ReactIR vessel and cooled to $-50{ }^{\circ} \mathrm{C}$ for $20 \mathrm{~min}$. In a separate $5 \mathrm{~mL}$ Kimble vial, ester 5 (0.315 g, $1.1 \mathrm{mmol})$ was dissolved in $1.05 \mathrm{~mL}$ THF and $100 \mu \mathrm{L}$ was injected into the IR vessel. The spectra were collected every $30 \mathrm{~s}$. The $\mathrm{C}=\mathrm{O}$ absorbance $\left(1732 \mathrm{~cm}^{-1}\right)$ decreased to the baseline with the appearance of a new absorbance at $1620 \mathrm{~cm}^{-1}$. The pseudo-first-order rate constant was $16 \pm 1 \times 10^{-3} \mathrm{~s}^{-1}$. An additional $400 \mu \mathrm{L}$ of the solution of ester 5 was injected and the reaction was quenched with $\mathrm{H}_{2} \mathrm{O}$ when the $\mathrm{C}=\mathrm{O}$ absorbance had disappeared.

Chromatographic isolation of the products $\left(95 / 5 \mathrm{CH}_{2} \mathrm{Cl}_{2} / \mathrm{CH}_{3} \mathrm{OH}\right)$ provided esters $\mathbf{4}$ and $\mathbf{5}$ in a 28 and $56 \%$ yield, respectively. 


\section{LiHMDS-mediated enolization of free base 6.}

LiHMDS (0.201 g, $1.2 \mathrm{mmol}$ ) was dissolved in $7.4 \mathrm{~mL}$ THF and $2.25 \mathrm{~mL}$ toluene. The solution was placed in a ReactIR vessel and cooled to $0{ }^{\circ} \mathrm{C}$ for $20 \mathrm{~min}$. Ester $(R)-6(0.117 \mathrm{~g}, 1.0 \mathrm{mmol})$ was injected into the IR vessel and the spectrum was acquired within $20 \mathrm{~s}$. The absence of a $\mathrm{C}=\mathrm{O}$ absorbance indicates complete enolization. The enolate $\mathrm{C}=\mathrm{C}$ absorbance was at $1618 \mathrm{~cm}^{-1}$.

\section{LiHMDS-mediated enolization and cyclization of 7/8.}

The reaction of LiHMDS with $7(\bullet)$ and $8(\boldsymbol{v})$ is instantaneous in $9.0 \mathrm{M} \mathrm{THF} /$ toluene at $0{ }^{\circ} \mathrm{C}$.

Therefore, the formation of lactams $14(-)$ and $15(\bullet)$ was monitored by incremental addition of LiHMDS equivalents with subsequent GC analysis post-quenching. The reaction yields a final ratio of lactams $14: 15$ of $7.5: 1$ using an initial ester $7: 8$ ratio of $1.5: 1$.

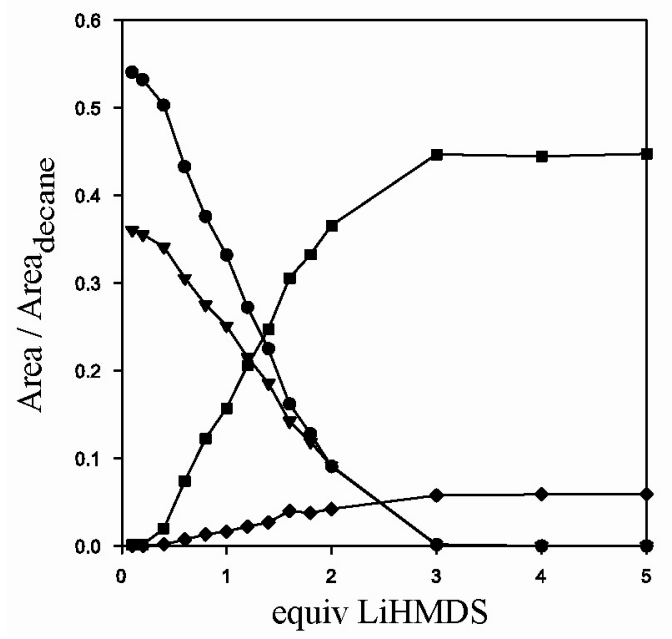




\section{In-situ formation of lactam 14 with LiHMDS/rac-9.}
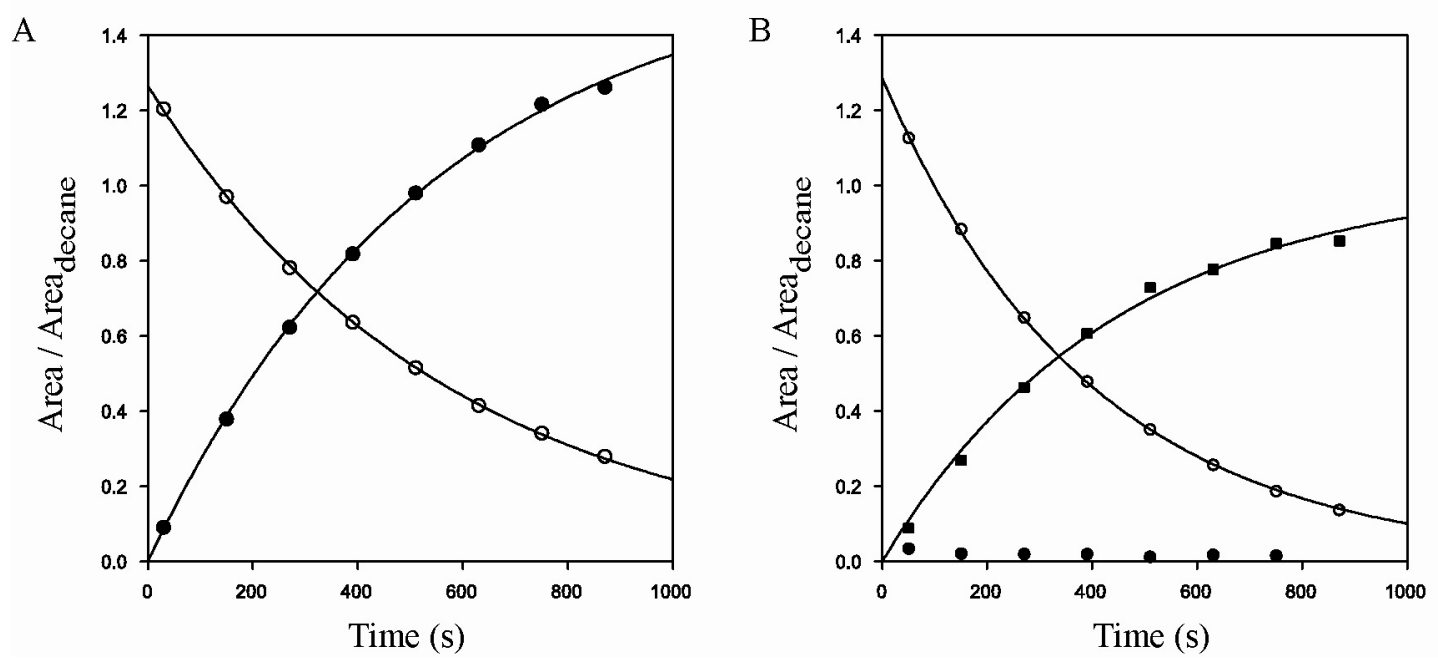

(A) Enolate rac-9 (0.074 g, $0.6 \mathrm{mmol})$ was dissolved in $1.51 \mathrm{~mL}$ THF and $0.42 \mathrm{~mL}$ toluene at 0 ${ }^{\circ} \mathrm{C}$. A stock solution of $\mathrm{BnBr}(50 \mu \mathrm{L}, 0.2 \mathrm{M} \mathrm{BnBr} / 0.1 \mathrm{M}$ decane in toluene $)$ was injected and the reaction monitored by $\mathrm{GC}$ analysis of $\mathrm{H}_{2} \mathrm{O}$-quenched samples ( $\mathrm{Et}_{2} \mathrm{O}$ extract). The pseudo-firstorder rate constant for the loss of $\mathrm{BnBr}(\circ)$ is $1.760 \pm 0.001 \times 10^{-3} \mathrm{~s}^{-1}$ and for the appearance of ester $7(\bullet)$ is $1.8 \pm 0.1 \times 10^{-3} \mathrm{~s}^{-1}$.

(B) Enolate rac-9 $(0.074 \mathrm{~g}, 0.6 \mathrm{mmol})$ was dissolved in $1.51 \mathrm{~mL}$ THF and $0.42 \mathrm{~mL}$ toluene at 0

${ }^{\circ} \mathrm{C}$. LiHMDS $(0.010 \mathrm{~g}, 0.06 \mathrm{mmol})$ was added to the vial. A stock solution of $\mathrm{BnBr}(50 \mu \mathrm{L}, 0.2$ $\mathrm{M} \mathrm{BnBr} / 0.1 \mathrm{M}$ decane in toluene) was injected and the reaction monitored by GC analysis of $\mathrm{H}_{2} \mathrm{O}$-quenched aliquots ( $\mathrm{Et}_{2} \mathrm{O}$ extract). The pseudo-first-order rate constant for the loss of $\mathrm{BnBr}$ (॰) is $2.55 \pm 0.02 \times 10^{-3} \mathrm{~s}^{-1}$ and for the appearance of lactam $14(\mathbf{a})$ is $2.3 \pm 0.2 \times 10^{-3} \mathrm{~s}^{-1}$. 


\section{Formation of stilbene 13 from LiHMDS/3.}

LiHMDS (0.033 g, $0.2 \mathrm{mmol})$ was weighed into a $5 \mathrm{~mL}$ round-bottom flask. THF $(1.46 \mathrm{~mL})$ and toluene $(0.51 \mathrm{~mL})$ were added to the flask. The solution was cooled to $0{ }^{\circ} \mathrm{C}$ for $10 \mathrm{~min}$. A stock solution of 3 ( $32 \mu \mathrm{L}, 0.6 \mathrm{M} \mathrm{3} / 0.3 \mathrm{M}$ decane in toluene) was added to the flask and the reaction was monitored over $5 \mathrm{~min}$ via GC analysis of quenched aliquots. The quench consisted of $3 \mathrm{M}$ $\mathrm{HCl} / 2 \mathrm{M} \mathrm{NaCl}$ in $\mathrm{H}_{2} \mathrm{O}(0.5 \mathrm{~mL})$ and $\mathrm{Et}_{2} \mathrm{O}(1.0 \mathrm{~mL})$. The pseudo-first-order rate constant for the reaction of LiHMDS with 3 to produce stilbene 13 was $8 \pm 1 \times 10^{-3} \mathrm{~s}^{-1}$ (average of two runs).

\section{GC analysis of LiHMDS with BnBr.}

LiHMDS (0.033 g, $0.2 \mathrm{mmol})$ was weighed into a $5 \mathrm{~mL}$ round-bottom flask. THF $(1.46 \mathrm{~mL})$ and toluene $(0.51 \mathrm{~mL})$ were added to the flask. The solution was cooled to $0{ }^{\circ} \mathrm{C}$ for $10 \mathrm{~min}$. A stock solution of $\mathrm{BnBr}(32 \mu \mathrm{L}, 0.6 \mathrm{M} \mathrm{BnBr} / 0.3 \mathrm{M}$ decane in toluene) was added to the flask and the reaction was monitored over $1 \mathrm{~h}$ via $\mathrm{GC}$ analysis of quenched aliquots. The quench consisted of $3 \mathrm{M} \mathrm{HCl} / 2 \mathrm{M} \mathrm{NaCl}$ in $\mathrm{H}_{2} \mathrm{O}(0.5 \mathrm{~mL})$ and $\mathrm{Et}_{2} \mathrm{O}(1.0 \mathrm{~mL})$. There was no change in the $\mathrm{GC}$ area ratio of $\mathrm{BnBr} / \mathrm{dec}$ ane over $1 \mathrm{~h}$.

\section{Table of $\boldsymbol{k}_{\text {rel }}$ for alkylations.}

\begin{tabular}{cccc}
\hline Ester & Electrophile & $k_{\text {obsd }}\left(\mathrm{s}^{-1}\right)$ & $k_{\text {rel }}$ \\
\hline$(R)-\mathbf{6}$ & $\mathrm{BnBr}^{a}$ & $1.75 \pm 0.3 \times 10^{-3}$ & 2.2 \\
$r a c-6$ & $\mathrm{BnBr}^{a}$ & $1.02 \pm 0.3 \times 10^{-3}$ & 1.3 \\
$\mathbf{2}$ & $\mathrm{BnBr}^{b}$ & $0.8 \pm 0.1 \times 10^{-3}$ & 1.0 \\
$(R)-\mathbf{6}$ & $\mathbf{3}^{a}$ & $14.6 \pm 0.6 \times 10^{-3}$ & 18.7 \\
$r a c-6$ & $\mathbf{3}^{a}$ & $8.3 \pm 0.0 \times 10^{-3}$ & 10.4 \\
$\mathbf{2}$ & $\mathbf{3}^{b}$ & $9.2 \pm 0.1 \times 10^{-3}$ & 11.5 \\
\hline
\end{tabular}

a. 9.0 M THF/toluene, 0.10 M LiHMDS, 0.12 M ester 6, and 0.01 M electrophile.

b. 9.0 M THF/toluene, 0.20 M LiHMDS, 0.11 M salt 2, and 0.01 M electrophile. 


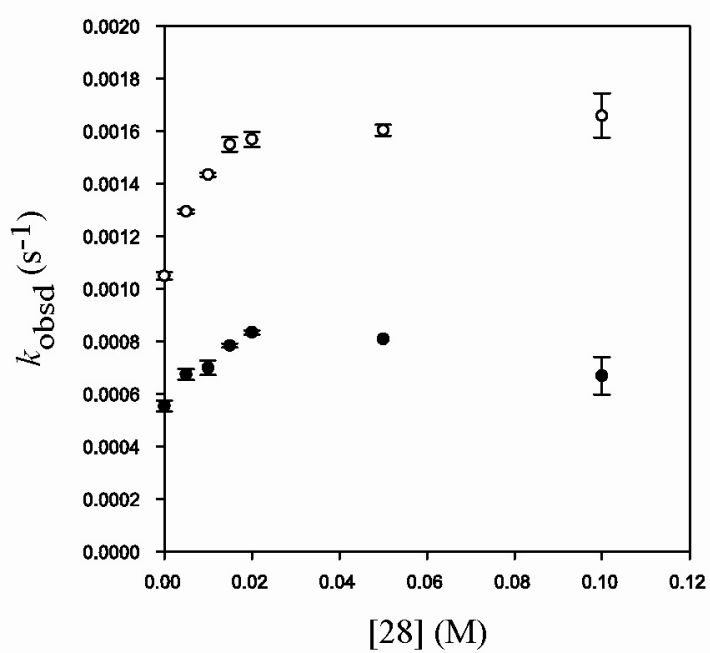

19. Plot of $k_{\text {obsd }}$ versus [28] in $6.0 \mathrm{M} \mathrm{THF} /$ toluene for the alkylation of $(R)-9(\circ, 0.10 \mathrm{M})$ and $\mathrm{rac}$ $9(\bullet, 0.10 \mathrm{M})$ with $\mathrm{BnBr}(0.01 \mathrm{M})$ at $0{ }^{\circ} \mathrm{C}$.

20. Table of data for plot in Figure 19.

\begin{tabular}{ccc}
\hline$[\mathbf{2 8}](\mathrm{M})$ & $(R)-9 k_{\text {obsd }}\left(\mathrm{s}^{-1}\right)$ & $r a c-9 k_{\text {obsd }}\left(\mathrm{s}^{-1}\right)$ \\
\hline 0 & $10.5 \pm 0.1 \times 10^{-4}$ & $5.5 \pm 0.2 \times 10^{-4}$ \\
0.005 & $13.0 \pm 0.1 \times 10^{-4}$ & $6.8 \pm 0.2 \times 10^{-4}$ \\
0.01 & $14.4 \pm 0.1 \times 10^{-4}$ & $7.0 \pm 0.3 \times 10^{-4}$ \\
0.015 & $15.5 \pm 0.3 \times 10^{-4}$ & $7.8 \pm 0.1 \times 10^{-4}$ \\
0.02 & $15.7 \pm 0.3 \times 10^{-4}$ & $8.4 \pm 0.1 \times 10^{-4}$ \\
0.05 & $16.1 \pm 0.2 \times 10^{-4}$ & $8.1 \pm 0.0 \times 10^{-4}$ \\
0.10 & $16.6 \pm 0.8 \times 10^{-4}$ & $6.7 \pm 0.7 \times 10^{-4}$ \\
\hline
\end{tabular}




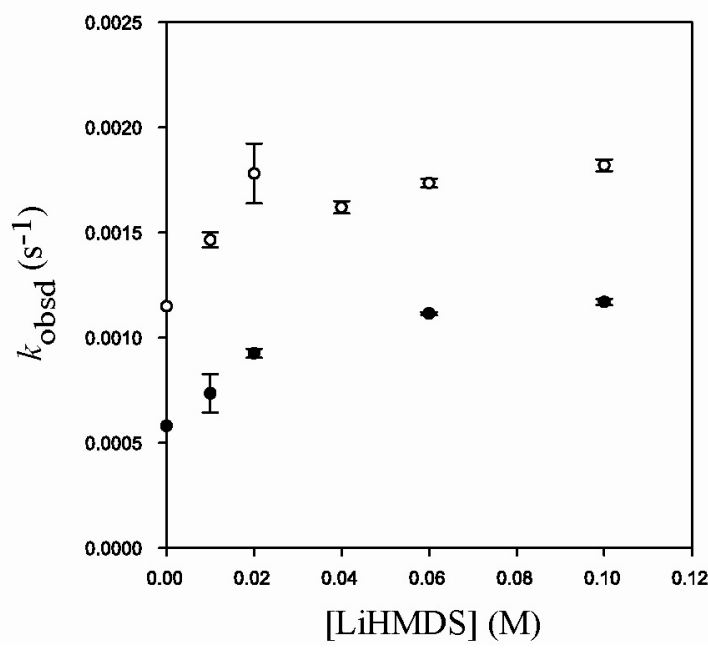

21. Plot of $k_{\text {obsd }}$ versus [LiHMDS] in $6.0 \mathrm{M} \mathrm{THF/toluene} \mathrm{for} \mathrm{the} \mathrm{alkylation} \mathrm{of}(R)-9(\circ, 0.10 \mathrm{M})$ and $\operatorname{rac}-9(\bullet, 0.10 \mathrm{M})$ with $\mathrm{BnBr}(0.01 \mathrm{M})$ at $0{ }^{\circ} \mathrm{C}$.

22. Table of data for plot in Figure 21.

\begin{tabular}{ccc}
\hline [LiHMDS] (M) & $(R)-9 k_{\text {obsd }}\left(\mathrm{s}^{-1}\right)$ & rac-9 $k_{\mathrm{obsd}}\left(\mathrm{s}^{-1}\right)$ \\
\hline 0.09 & $10.7 \pm 0.1 \times 10^{-4}$ & $5.5 \pm 0.3 \times 10^{-4}$ \\
0.10 & $11.5 \pm 0.0 \times 10^{-4}$ & $5.8 \pm 0.0 \times 10^{-4}$ \\
0.11 & $14.7 \pm 0.4 \times 10^{-4}$ & $7.4 \pm 0.9 \times 10^{-4}$ \\
0.12 & $18 \pm 1 \times 10^{-4}$ & $9.2 \pm 0.2 \times 10^{-4}$ \\
0.16 & $16.2 \pm 0.3 \times 10^{-4}$ & $11.2 \pm 0.1 \times 10^{-4}$ \\
0.20 & $18.2 \pm 0.3 \times 10^{-4}$ & $11.7 \pm 0.1 \times 10^{-4}$ \\
\hline
\end{tabular}




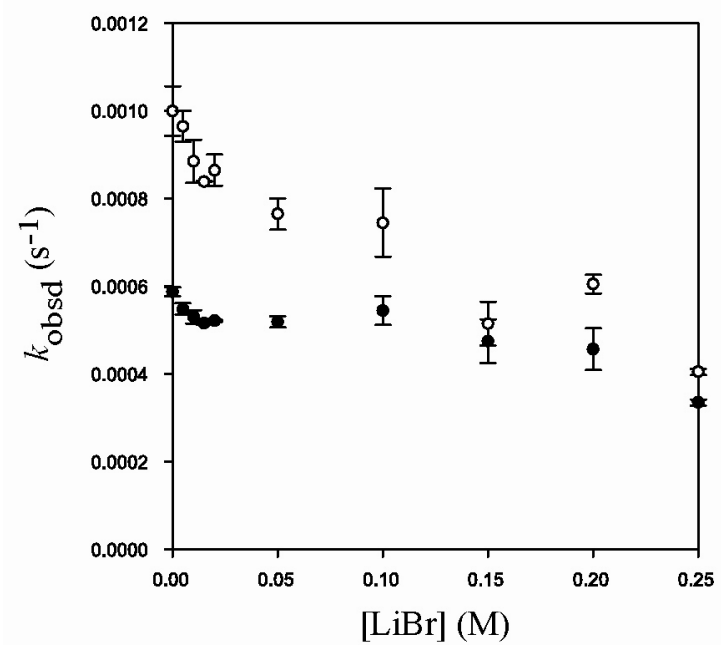

23. Plot of $k_{\text {obsd }}$ versus [LiBr] in 6.0 M THF/toluene for the alkylation of $(R)-9(\circ, 0.10 \mathrm{M})$ and $\operatorname{rac}-9(\bullet, 0.10 \mathrm{M})$, respectively, with $\operatorname{BnBr}(0.01 \mathrm{M})$ at $0{ }^{\circ} \mathrm{C}$.

24. Table of data for plot in Figure 23.

\begin{tabular}{ccc}
\hline$[\mathrm{LiBr}](\mathrm{M})$ & $(R)-9 k_{\text {obsd }}\left(\mathrm{s}^{-1}\right)$ & $r a c-9 k_{\mathrm{obsd}}\left(\mathrm{s}^{-1}\right)$ \\
\hline 0.00 & $10.0 \pm 0.5 \times 10^{-4}$ & $5.9 \pm 0.1 \times 10^{-4}$ \\
0.005 & $9.6 \pm 0.4 \times 10^{-4}$ & $5.5 \pm 0.1 \times 10^{-4}$ \\
0.01 & $8.8 \pm 0.5 \times 10^{-4}$ & $5.3 \pm 0.1 \times 10^{-4}$ \\
0.015 & $8.39 \pm 0.01 \times 10^{-4}$ & $5.12 \pm 0.03 \times 10^{-4}$ \\
0.02 & $8.6 \pm 0.4 \times 10^{-4}$ & $5.22 \pm 0.02 \times 10^{-4}$ \\
0.05 & $7.6 \pm 0.4 \times 10^{-4}$ & $5.2 \pm 0.1 \times 10^{-4}$ \\
0.10 & $7.4 \pm 0.8 \times 10^{-4}$ & $5.5 \pm 0.3 \times 10^{-4}$ \\
0.15 & $5.2 \pm 0.5 \times 10^{-4}$ & $4.8 \pm 0.5 \times 10^{-4}$ \\
0.20 & $6.0 \pm 0.2 \times 10^{-4}$ & $4.6 \pm 0.5 \times 10^{-4}$ \\
0.25 & $4.05 \pm 0.07 \times 10^{-4}$ & $3.35 \pm 0.07 \times 10^{-4}$ \\
\hline
\end{tabular}




\section{Single-Crystal X-ray Structure Data}

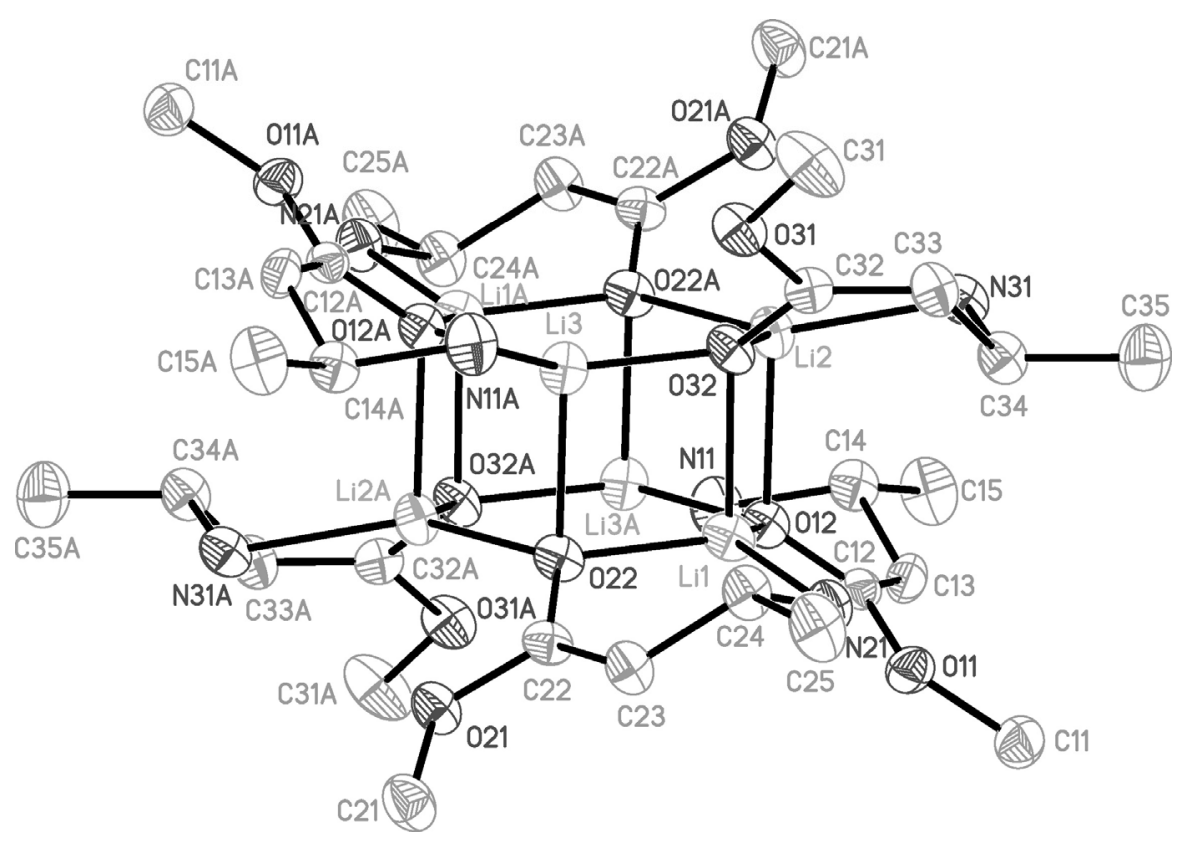

25. Crystals of $\mathrm{rac}-\mathbf{9}$ were obtained from a $0.20 \mathrm{M}$ enolate solution in $9.0 \mathrm{M} \mathrm{THF} /$ toluene held at $-20{ }^{\circ} \mathrm{C}$ over $24 \mathrm{~h}$. Single crystals suitable for X-ray diffraction were coated with polyisobutylene oil in a glovebox and were quickly transferred to the goniometer head of a Siemens SMART $(\lambda=$ $0.71073 \mathrm{~A} \mathrm{~T}=173 \mathrm{~K}$ ). The crystal belongs to the space group P1(bar). 1818 frames were collected using 0.3 deg. omega scans $\left(2 \theta_{\max }=46.52^{\circ}\right)$. The data were processed with Bruker SAINT and SADABS programs to yield a total of 6120 unique reflections $(\mathrm{R}(\mathrm{int})=0.0575)$. The structure was solved using direct method (SHELXS) completed by subsequent Fourier synthesis and refined by full-matrix least-squares procedures (SHELXL). At final convergence, $\mathrm{R}(1)=0.0665$ for $3433 \mathrm{Fo}>4 \operatorname{sig}(\mathrm{Fo})$ and GOF $=0.949$ for 772 parameters. For the crystal structure data and refinement details, see McNeil, A. J.; Toombes, G. E. S.; Chandramouli, S. V.; Vanasse, B. J.; Ayers, T. A.; O’Brien, M. K.; Lobkovsky, E.; Gruner, S. M.; Marohn, J. A.; Collum, D. B. J. Am. Chem. Soc. 2004, 126, 5938-5939 (CCDC 231371). 


\section{Powder Diffraction Data}

26. Powder X-ray diffraction data was collected on a Scintag XDS-2000 with $\mathrm{Cu} \mathrm{K}_{\alpha}$ radiation and a liquid nitrogen cooled Ge solid-state detector. The sample was packed inside a glove box under argon atmosphere in a holder for air-sensitive samples to protect it from atmospheric moisture. The pattern was measured over the angular range 2 to $50^{\circ} 2 \theta$ at a rate of $0.1 \% \mathrm{~min}$. The data were fitted with the Le Bail method, using lattice constants from single crystal data as a starting point. The lattice constants refined to values of $\mathrm{a}=13.7655 \AA, \mathrm{b}=13.2951 \AA, \mathrm{c}=$ $13.8038 \AA, \alpha=11.765^{\circ}, \beta=92.50^{\circ}$ and $\gamma=103.72^{\circ}$. (For reference, see Le Bail, A.; Duroy, H.; Fourquet, J. L. Mater. Res. Bull. 1988, 23, 447-452 and Rodriguez-Carvajal, J. FULLPROF: A Program for Rietveld Refinement and Pattern Matching Analysis. Abstracts of the Satellite Meeting on Powder Diffraction of the X $V^{\text {th }}$ Congress of the IUCr, Toulouse, France, 1990; p 127.)

27. Powder diffraction pattern for $\mathrm{rac}_{\mathbf{- 9}} \mathbf{9}$ and the comparison of calculated fit to the data is shown.

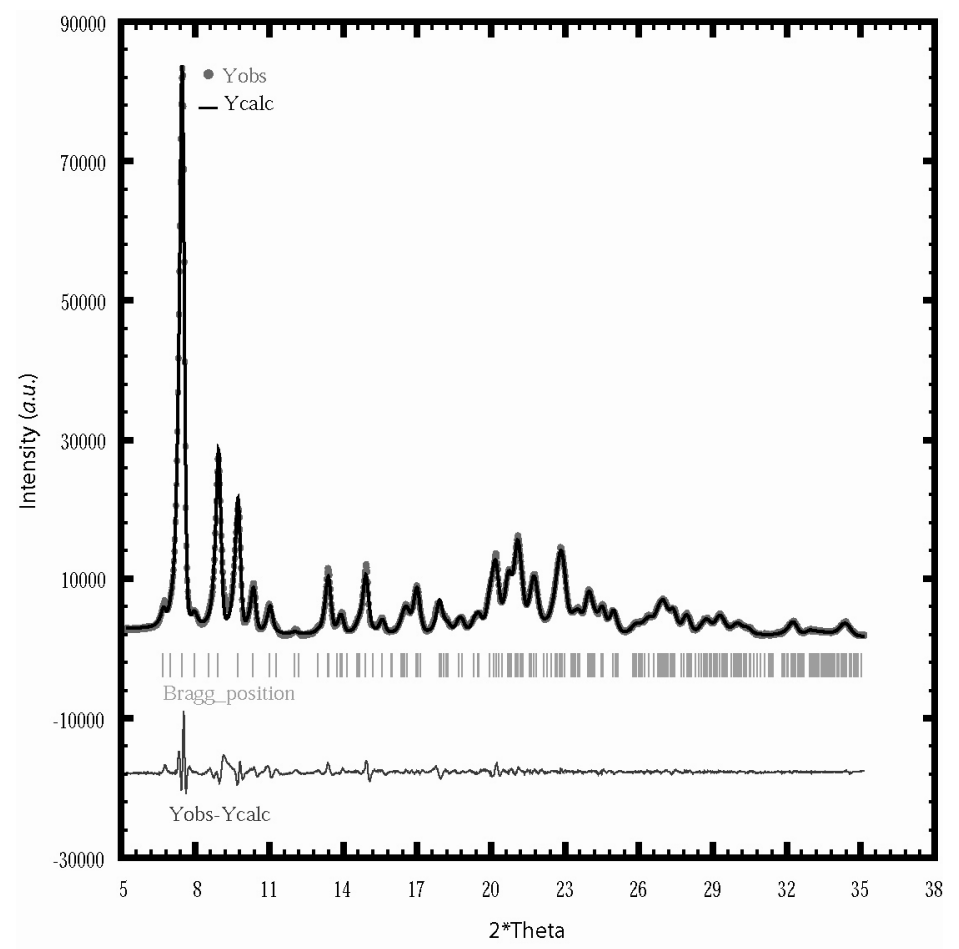




\section{Small-Angle X-ray Scattering Data}

28. Experimental Details. Small angle X-ray scattering (SAXS) data were obtained using an RU-H3R Cu rotating anode X-ray generator directed through a nickel filter and collimated with orthogonal Franks mirrors $\left(\mathrm{d}_{\max }=400 \AA\right)$. Tantalum slits at the sample stage trim the beam to approximately $1 \mathrm{~mm} \times 1 \mathrm{~mm}$ with an average flux of $4 \times 10^{7} \mathrm{X}$-rays per second. Sample temperature was monitored with a $100 \Omega$ platinum RTD sensor and regulated with a water-cooled Peltier controller operating within the vacuum beam path. Sample temperature was maintained at $0{ }^{\circ} \mathrm{C}$. Scattering path lengths from $10 \mathrm{~cm}$ to $100 \mathrm{~cm}$ were used to measure a wide range of sizes. Diffraction patterns were recorded (multiple exposures integrated to give 1-2 hours of total time) with a home-built CCD detector. The detector-sample distance was calibrated with powder patterns of silver stearate and silver behenate. Samples were flame-sealed in $1 \mathrm{~mm}$ glass X-ray capillary.

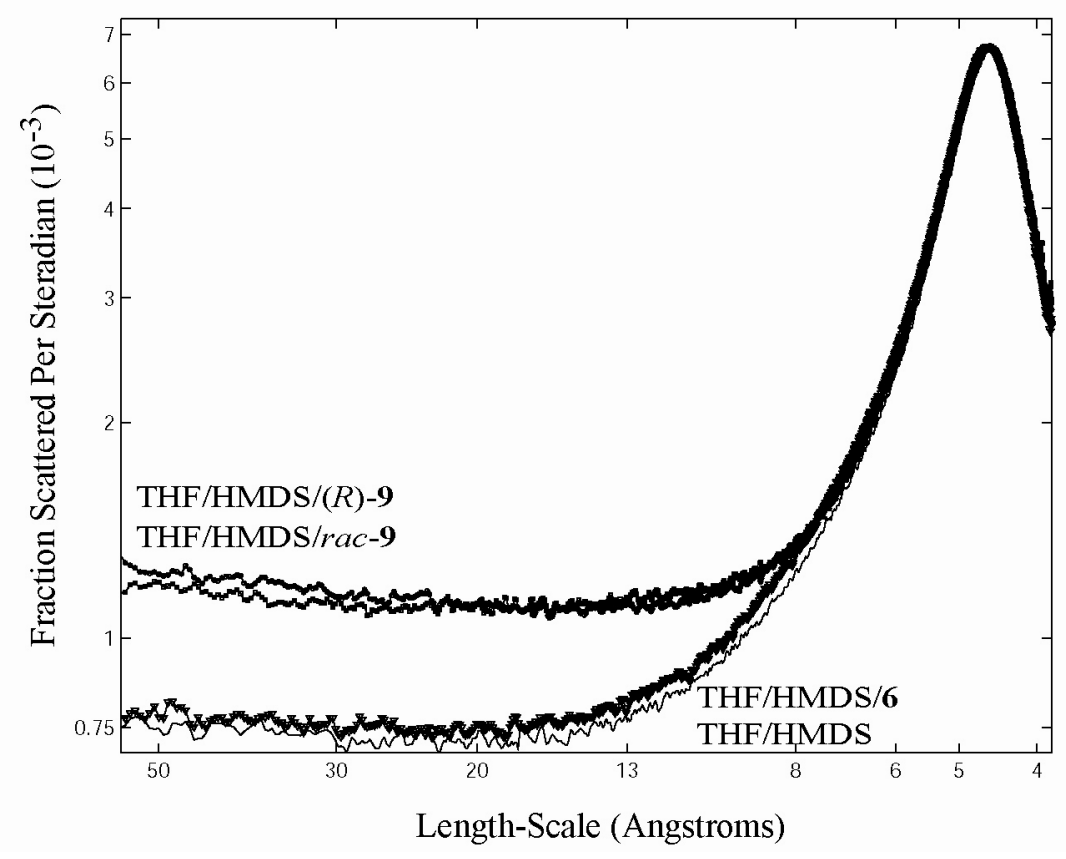

\section{Plot of the scattering intensity (I) as a function of momentum transfer (s). Typical} concentrations were $12 \mathrm{M}$ THF, $0.5 \mathrm{M}$ HMDS, and $0.5 \mathrm{M} 6$ or 9 . The THF contributes a strong peak at $4.56 \pm 0.25 \AA$ corresponding to the mean THF-THF distance in solution. When the enolate is added to the solution, extra scattering occurs at longer length-scales giving clear indication that the enolate forms assemblies that are measurably larger than the monomer (6). No new features were observed for length-scales longer than $50 \AA$. 


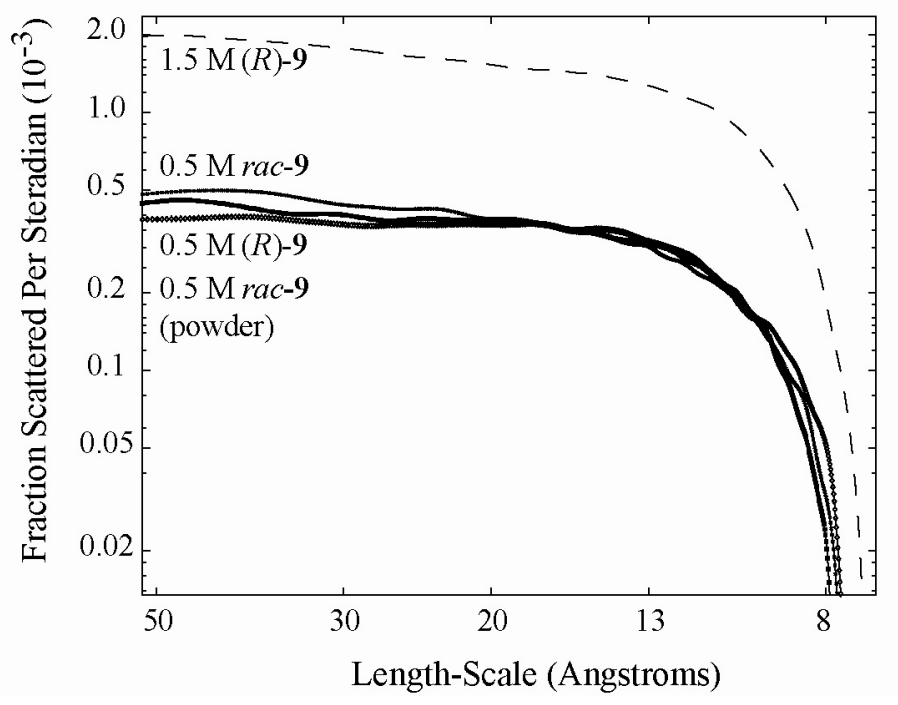

30. Plot of the additional scattering as a function of momentum transfer. Solvation effects make it difficult to unambiguously determine the size of the scattering objects. However, the objects should be between 7 and $14 \AA$, as this is the length over which the scattering changes markedly. The crystal structure of the hexamer allows us to compute its size in solution. In vacuum, the diameter is $8.88 \AA$, but the apparent diameter can vary from 6.5 and $13.1 \AA$ depending on how the hexamer is solvated. Although the observed scattering is not that of ideal, isolated particles in solution, the apparent length-scale is consistent with a solution of hexamers. 


\section{Solubility Studies Using In-Situ IR}

31. Calibration plot. Using 2,2,4,4-tetramethyl-3-pentanone as an internal standard $(C=O, 1684$ $\mathrm{cm}^{-1}$ ), a calibration plot to determine [enolate] based on the ratio of absorbance (enolate) / absorbance (standard) was constructed in $9.0 \mathrm{M} \mathrm{THF} /$ toluene at $0{ }^{\circ} \mathrm{C}$. The line depicts an unweighted least-squares fit to $\left[\mathrm{Abs}_{\text {rac }-9} / \mathrm{Abs}_{\text {standard }}\right]=\mathrm{m}[\mathrm{rac}-\mathbf{9}]+\left[\mathrm{Abs}_{\mathrm{rac}-9} / \mathrm{Abs}_{\text {standard }}\right]_{\mathrm{o}}$ with $\mathrm{m}=$ $23.1 \pm 0.8$ and $\left[\mathrm{Abs}_{\text {rac }-9} / \mathrm{Abs}_{\text {standard }}\right]_{\mathrm{o}}=0.4 \pm 0.1$.

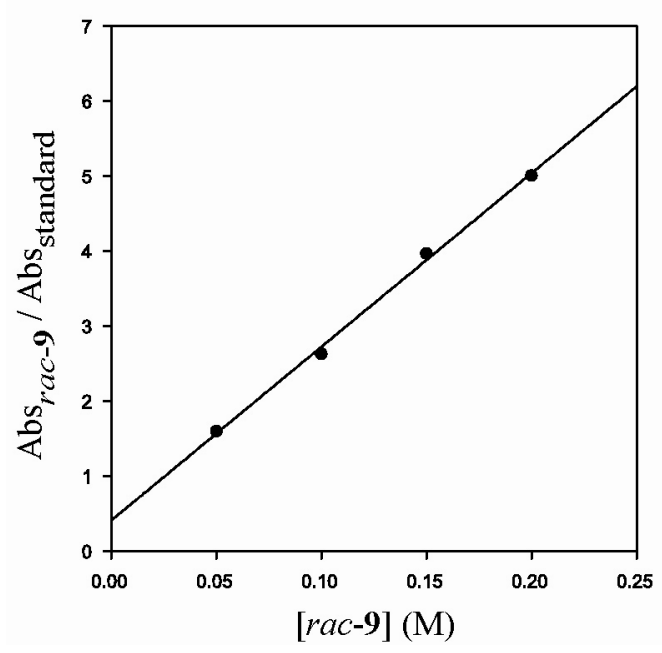


32. Supersaturation. Supersaturated solutions of $\mathrm{rac}-9$ in $9.0 \mathrm{M} \mathrm{THF} /$ toluene were monitored via in situ IR at $0{ }^{\circ} \mathrm{C}$ until equilibrium was reached. A representative example is described in more detail as follows: A solution of 0.87 M LiHMDS in 9.0 M THF/toluene was placed in the IR vessel and cooled to $0{ }^{\circ} \mathrm{C}$ for $20 \mathrm{~min}$. The baseline spectrum was acquired and the reaction started. Injecting $1.0 \mathrm{~g}$ of $\mathbf{6}$ into the IR vessel produced a $0.85 \mathrm{M}$ solution of $\mathrm{rac}-\mathbf{9}$ with complete enolization $\left(1620 \mathrm{~cm}^{-1}, \mathrm{C}=\mathrm{C}\right)$. The decrease of the $\mathrm{C}=\mathrm{C}$ absorbance over time was monitored until equilibrium was maintained. 2,2,4,4-tetramethyl-3-pentanone $(100 \mu \mathrm{L})$ was injected. The

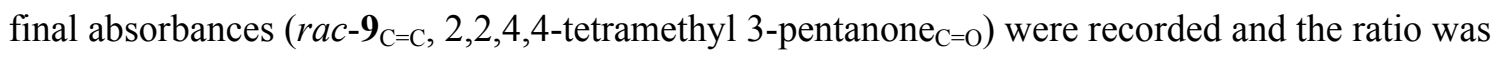
compared to the calibration plot to give a solution enolate concentration of $0.22 \mathrm{M}$.

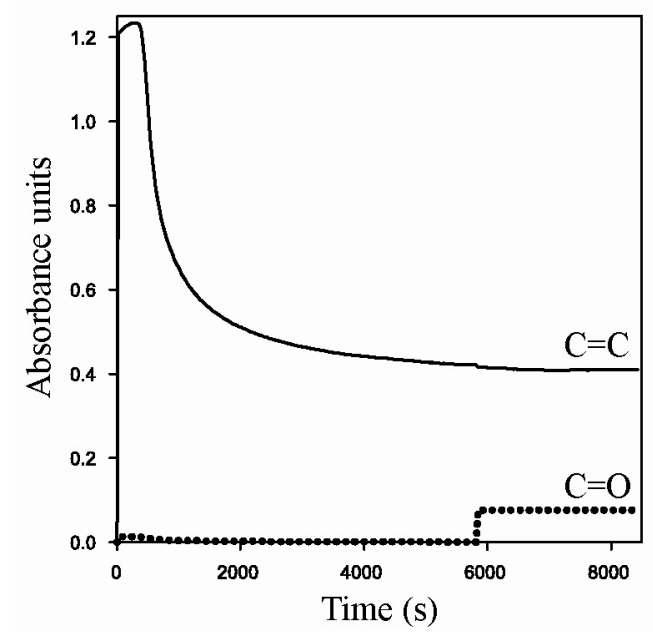

In a separate set of experiments, the absorbances for known concentrations of $\mathrm{rac}-\mathbf{9}$ were measured. The solubility was confirmed to be $0.22 \mathrm{M}$ through these studies as well.

Conversely, a $0.85 \mathrm{M}$ solution of $(R)-9$ was stable in $9.0 \mathrm{M} \mathrm{THF} /$ toluene at $0{ }^{\circ} \mathrm{C}$ for $7.5 \mathrm{~h}$. 
33. Variable-temperature studies. To further probe the solubility of $\mathrm{rac}-\mathbf{9}$, a variabletemperature IR study was undertaken. A representative example is described in more detail as follows: A solution of 0.57 M LiHMDS in 9.0 M THF/toluene was placed in the IR vessel and cooled to $0{ }^{\circ} \mathrm{C}$ for $20 \mathrm{~min}$. The baseline spectrum was acquired and the reaction started. Injecting $0.64 \mathrm{~g}$ of 6 into the IR vessel produced a $0.55 \mathrm{M}$ solution of $\mathrm{rac}-9$ with complete enolization $\left(1620 \mathrm{~cm}^{-1}, \mathrm{C}=\mathrm{C}\right) .2,2,4,4$-tetramethyl-3-pentanone $(100 \mu \mathrm{L})$ was injected. After $15 \mathrm{~min}$, the bath temperature was lowered to $-78{ }^{\circ} \mathrm{C}$ and a milky-white precipitate formed with a subsequent decrease in the $\mathrm{C}=\mathrm{C}$ intensity. After $20 \mathrm{~min}$, the bath temperature was raised to $0{ }^{\circ} \mathrm{C}$ and the precipitate re-dissolved indicating the low-temperature precipitate is a different polymorph than the precipitate obtained at $0{ }^{\circ} \mathrm{C}$. Within $2 \mathrm{~h}$ at $0{ }^{\circ} \mathrm{C}$, the enolate had again precipitated and equilibrium was established. From the ratio of absorbances at equilibrium, the solubility limit of rac-9 was determined to be $0.20 \mathrm{M}$ in $9.0 \mathrm{M} \mathrm{THF} /$ toluene at $0{ }^{\circ} \mathrm{C}$.

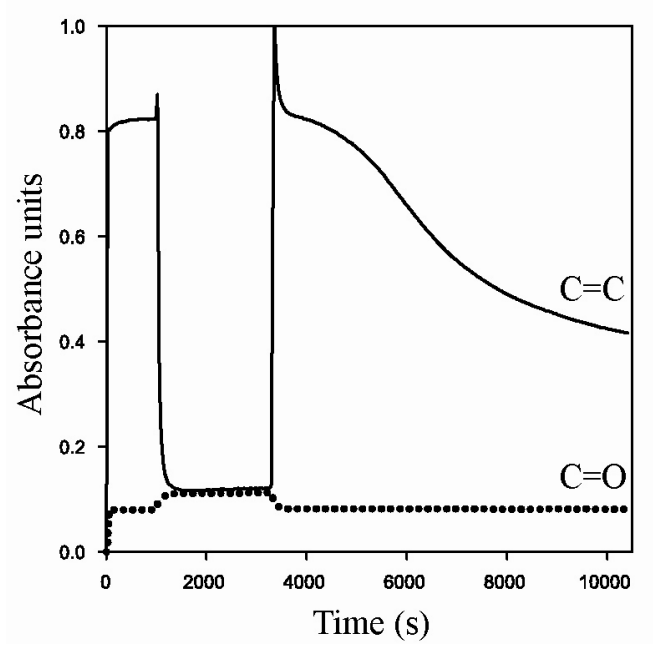

34. Isolation of solid rac-9. LiHMDS (1.21 g, $7.2 \mathrm{mmol})$ was dissolved in THF (7.9 mL) and cooled to $0{ }^{\circ} \mathrm{C}$. Ester $\mathrm{rac}-6(0.82 \mathrm{~g}, 7.0 \mathrm{mmol})$ was added drop wise to produce solution of $\mathrm{rac}-\mathbf{9}$. Within a few minutes, a milky-white precipitate was evident. The heterogeneous mixture was stirred for $4 \mathrm{~h}$ at $0{ }^{\circ} \mathrm{C}$ to establish equilibrium. After centrifugation at $0{ }^{\circ} \mathrm{C}$, the supernatant was removed via syringe $(6.5 \mathrm{~mL})$ and discarded. The remaining THF was removed under full vacuum at $0{ }^{\circ} \mathrm{C}$. The resulting powder was weighed in a glove box to yield $0.62 \mathrm{~g}$ of $\mathrm{rac}-9(70 \%$ yield). Assuming quantitative enolization of 6 to $\mathrm{rac}-\mathbf{9}$, a 70\% isolated yield of solid enolate corresponds to solution [ $\mathrm{rac}-9]$ of $0.19 \mathrm{M}$ in the supernatant. 


\section{${ }^{6}$ Li NMR Spectroscopic Studies}
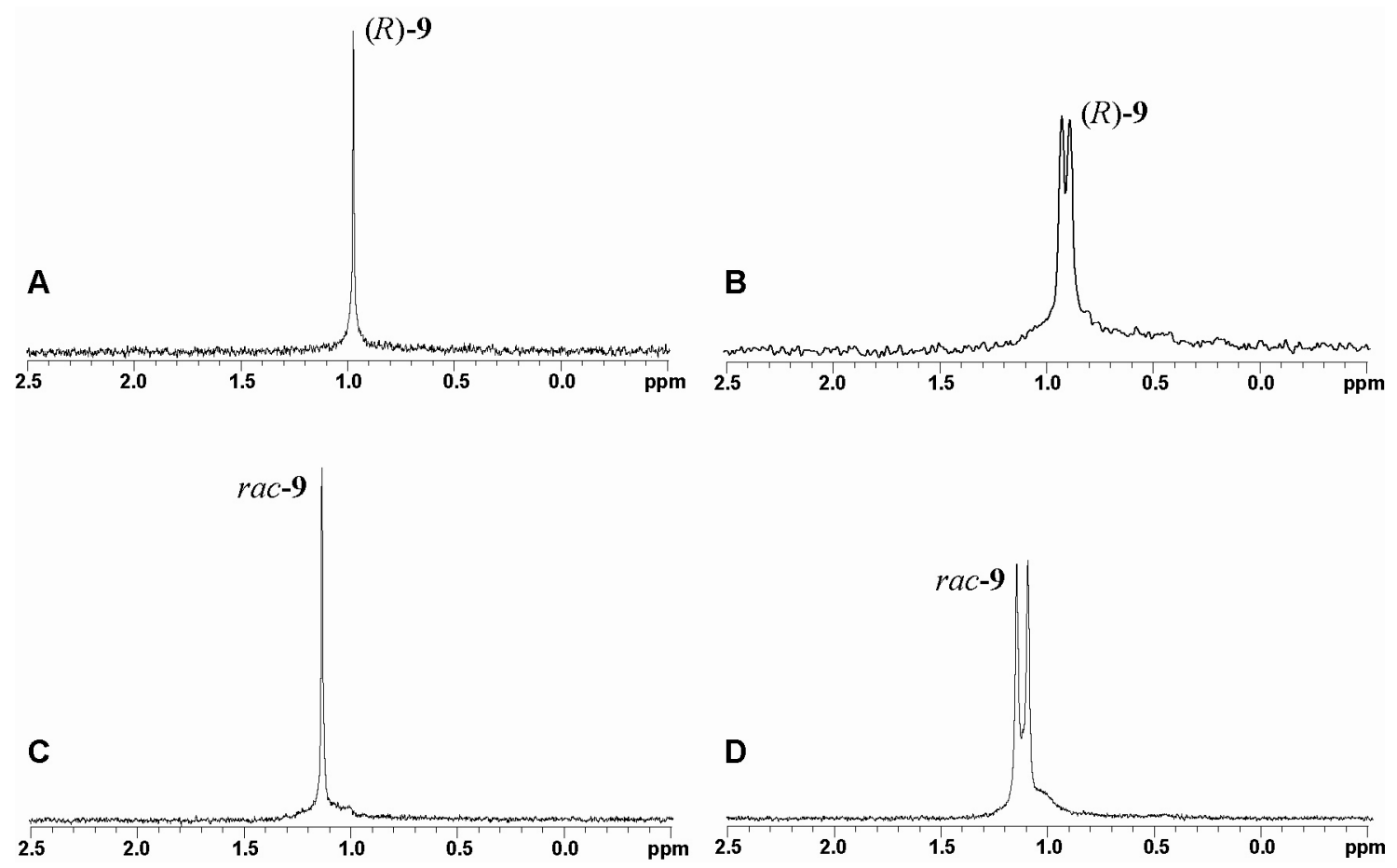

35. ${ }^{6} \mathrm{Li}$ NMR spectra recorded in $9.8 \mathrm{M} \mathrm{THF} /$ cyclopentane at $-90{ }^{\circ} \mathrm{C}:(\mathrm{A})\left[{ }^{6} \mathrm{Li}\right](R)-9(0.07 \mathrm{M})$;

(B) $\left[{ }^{6} \mathrm{Li},{ }^{15} \mathrm{~N}\right](S)-9(0.13 \mathrm{M})$; (C) $\left[{ }^{6} \mathrm{Li}\right] \mathrm{rac}-9(0.13 \mathrm{M})$; (D) $\left[{ }^{6} \mathrm{Li},{ }^{15} \mathrm{~N}\right] \mathrm{rac}-9(0.33 \mathrm{M})$. 

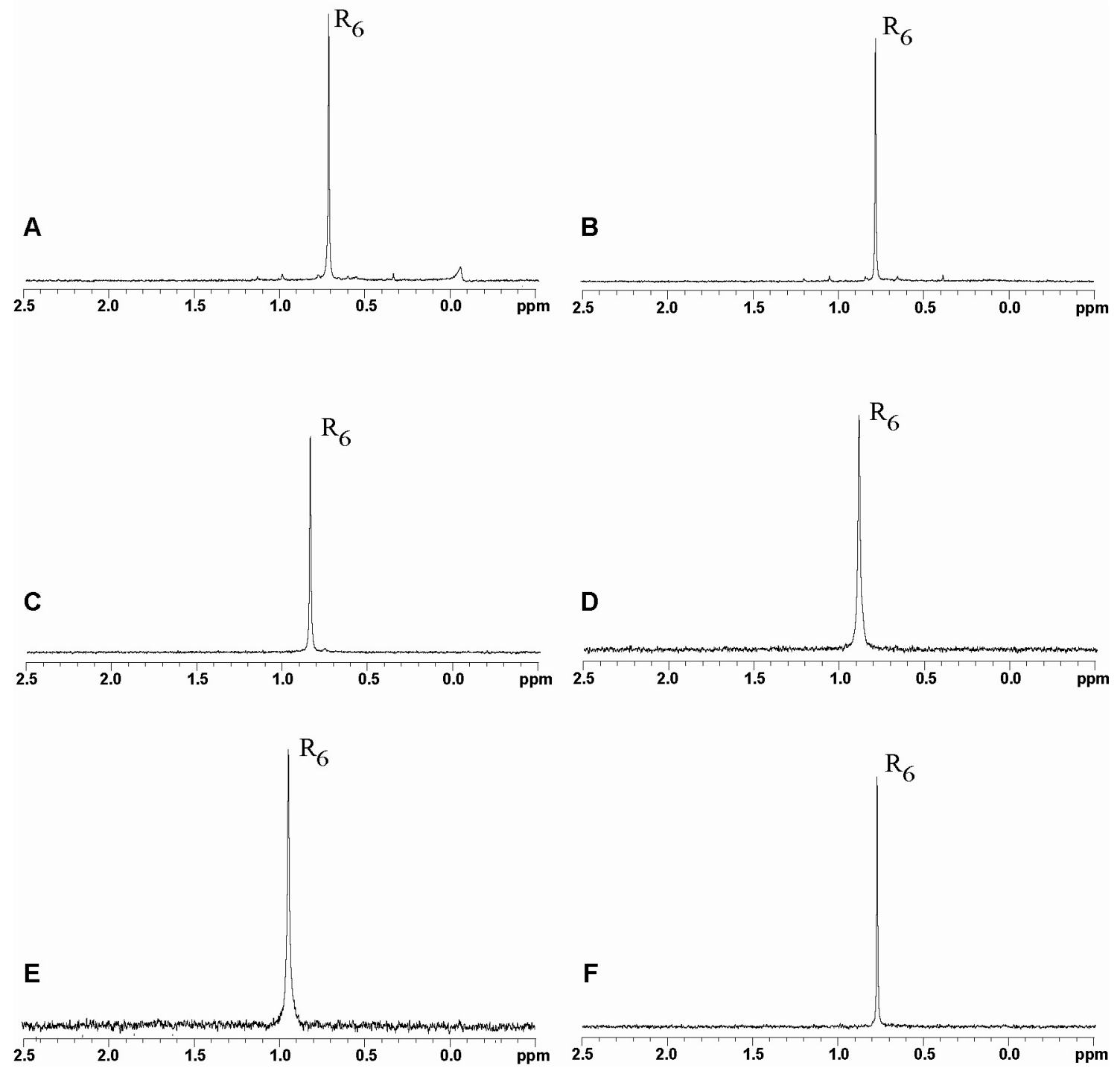

36. ${ }^{6} \mathrm{Li} \mathrm{NMR}$ spectra recorded on $\left[{ }^{6} \mathrm{Li}\right](R)-9(0.20 \mathrm{M})$ in $9.0 \mathrm{M}$ THF/toluene: (A) $-100{ }^{\circ} \mathrm{C}$; (B) $75{ }^{\circ} \mathrm{C}$; (C) $-50{ }^{\circ} \mathrm{C}$; (D) $-25{ }^{\circ} \mathrm{C}$; (E) $0{ }^{\circ} \mathrm{C}$; (F) $-90{ }^{\circ} \mathrm{C}$ after temperature series. 

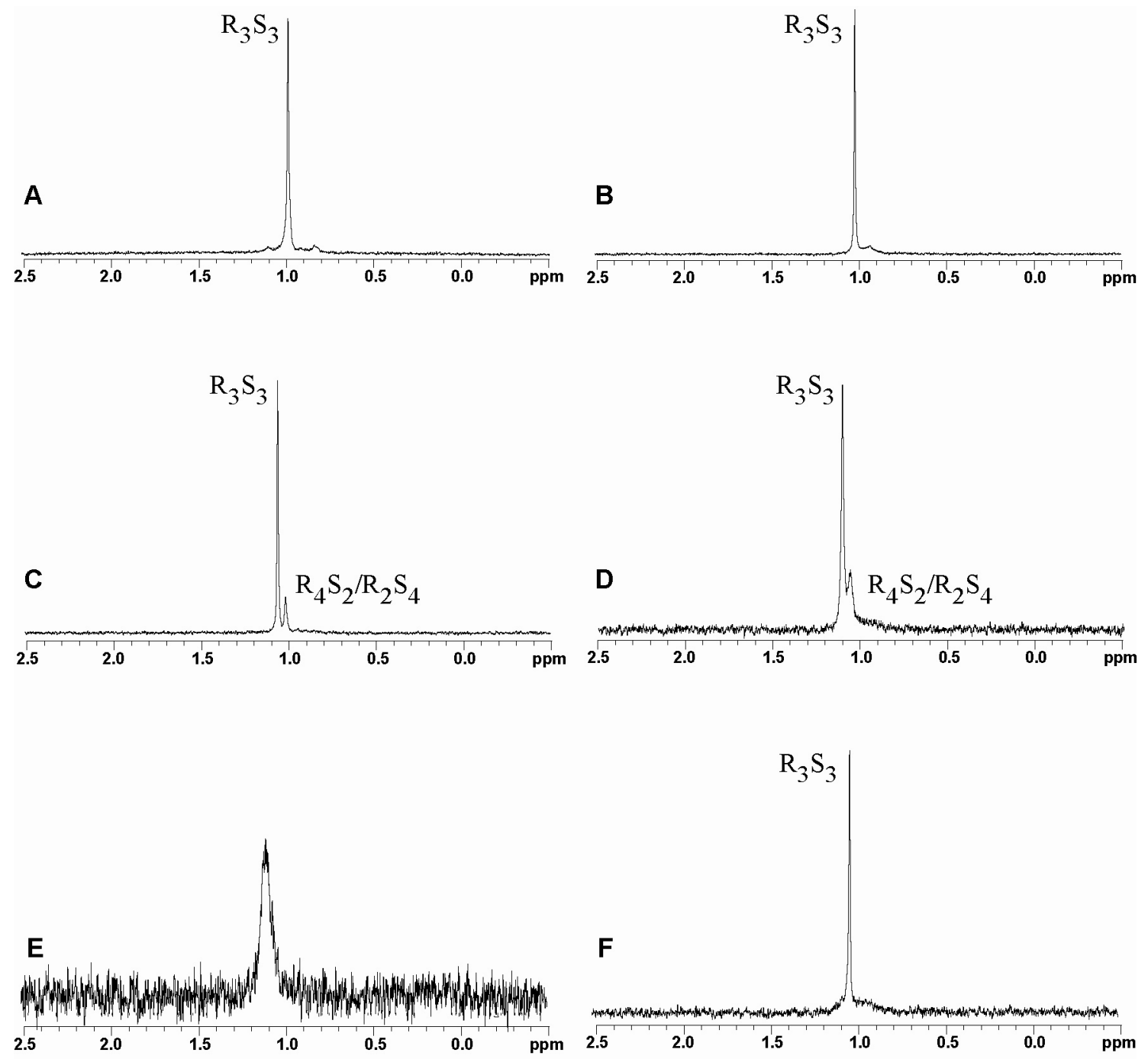

37. ${ }^{6} \mathrm{Li}$ NMR spectra recorded on $\left[{ }^{6} \mathrm{Li}\right] \mathrm{rac}-9(0.20 \mathrm{M})$ in $9.0 \mathrm{M}$ THF/toluene: $(\mathrm{A})-100{ }^{\circ} \mathrm{C}$; (B) $-75{ }^{\circ} \mathrm{C}$; (C) $-50{ }^{\circ} \mathrm{C}$; (D) $-25{ }^{\circ} \mathrm{C}$; (E) $0{ }^{\circ} \mathrm{C}$; (F) $-90{ }^{\circ} \mathrm{C}$ after temperature series. $\mathbf{R}_{\mathbf{n}} \mathbf{S}_{\mathrm{N}-\mathbf{n}} / \mathbf{R}_{\mathbf{N}-\mathbf{n}} \mathbf{S}_{\mathbf{n}}$ and $\mathbf{R}_{\mathbf{N}} / \mathbf{S}_{\mathbf{N}}$ refer to pairs of spectroscopically indistinguishable enantiomers. 

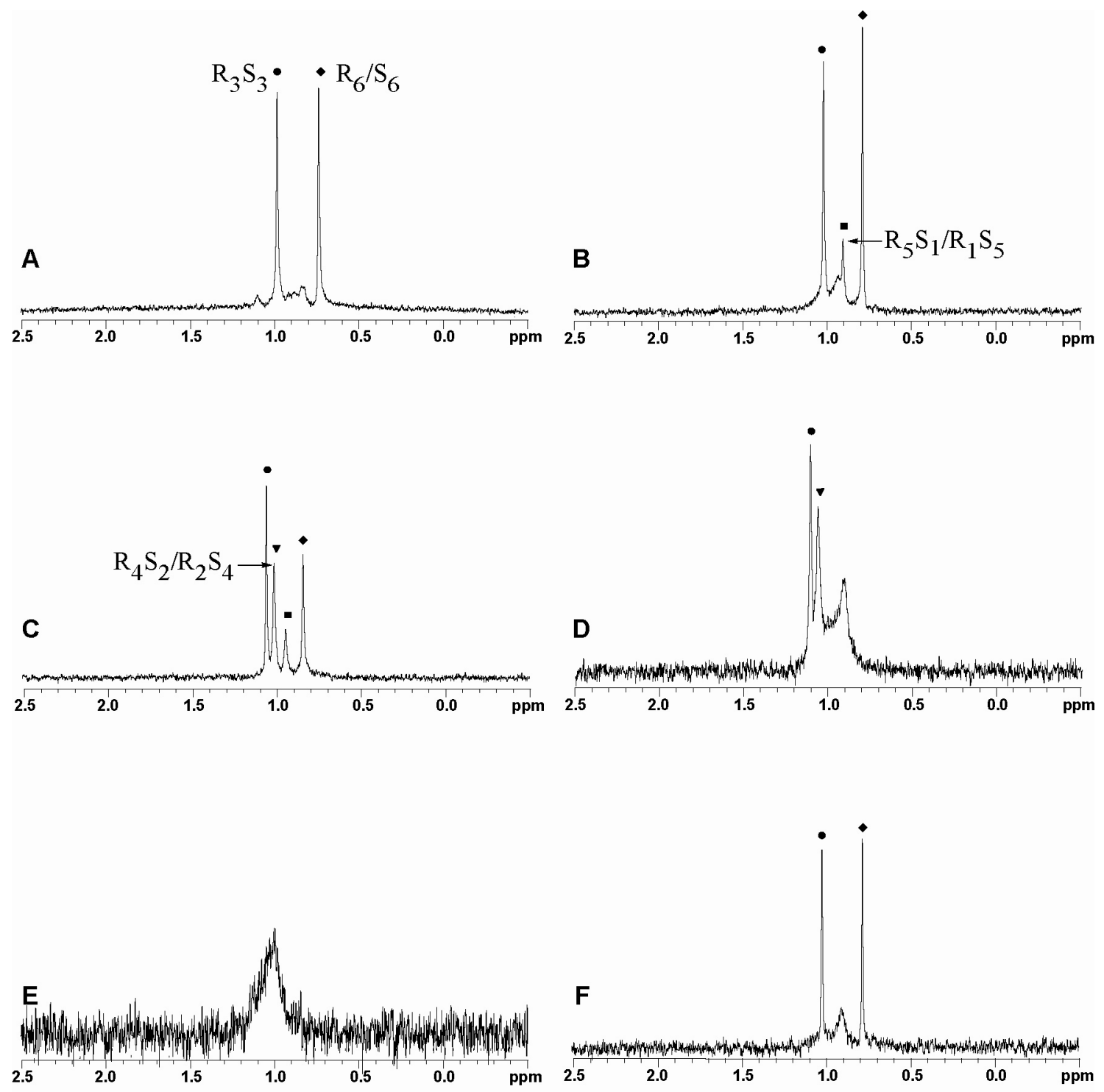

38. ${ }^{6} \mathrm{Li}$ NMR spectra recorded on a mixture of $\left[{ }^{6} \mathrm{Li}\right](R)-9$ and $\left[{ }^{6} \mathrm{Li}\right] \mathrm{rac}-9(50 \%$ ee $)$ in $9.0 \mathrm{M}$ THF/toluene: (A) $-100{ }^{\circ} \mathrm{C}$; (B) $-75{ }^{\circ} \mathrm{C}$; (C) $-50{ }^{\circ} \mathrm{C}$; (D) $-25{ }^{\circ} \mathrm{C}$; (E) $0{ }^{\circ} \mathrm{C}$; (F) $-90{ }^{\circ} \mathrm{C}$ after temperature series. $\mathbf{R}_{\mathbf{3}} \mathbf{S}_{\mathbf{3}}(\bullet) ; \mathbf{R}_{\mathbf{4}} \mathbf{S}_{2} / \mathbf{R}_{\mathbf{2}} \mathbf{S}_{\mathbf{4}}(\boldsymbol{\nabla}) ; \mathbf{R}_{5} \mathbf{S}_{\mathbf{1}} / \mathbf{R}_{\mathbf{1}} \mathbf{S}_{\mathbf{5}}(\boldsymbol{-}) ; \mathbf{R}_{\mathbf{6}} / \mathbf{S}_{\mathbf{6}}(\bullet) . \mathbf{R}_{\mathbf{n}} \mathbf{S}_{\mathrm{N}-\mathrm{n}} / \mathbf{R}_{\mathbf{N}-\mathrm{n}} \mathbf{S}_{\mathbf{n}}$ and $\mathbf{R}_{\mathbf{N}} / \mathbf{S}_{\mathbf{N}}$ refer to pairs of spectroscopically indistinguishable enantiomers. 

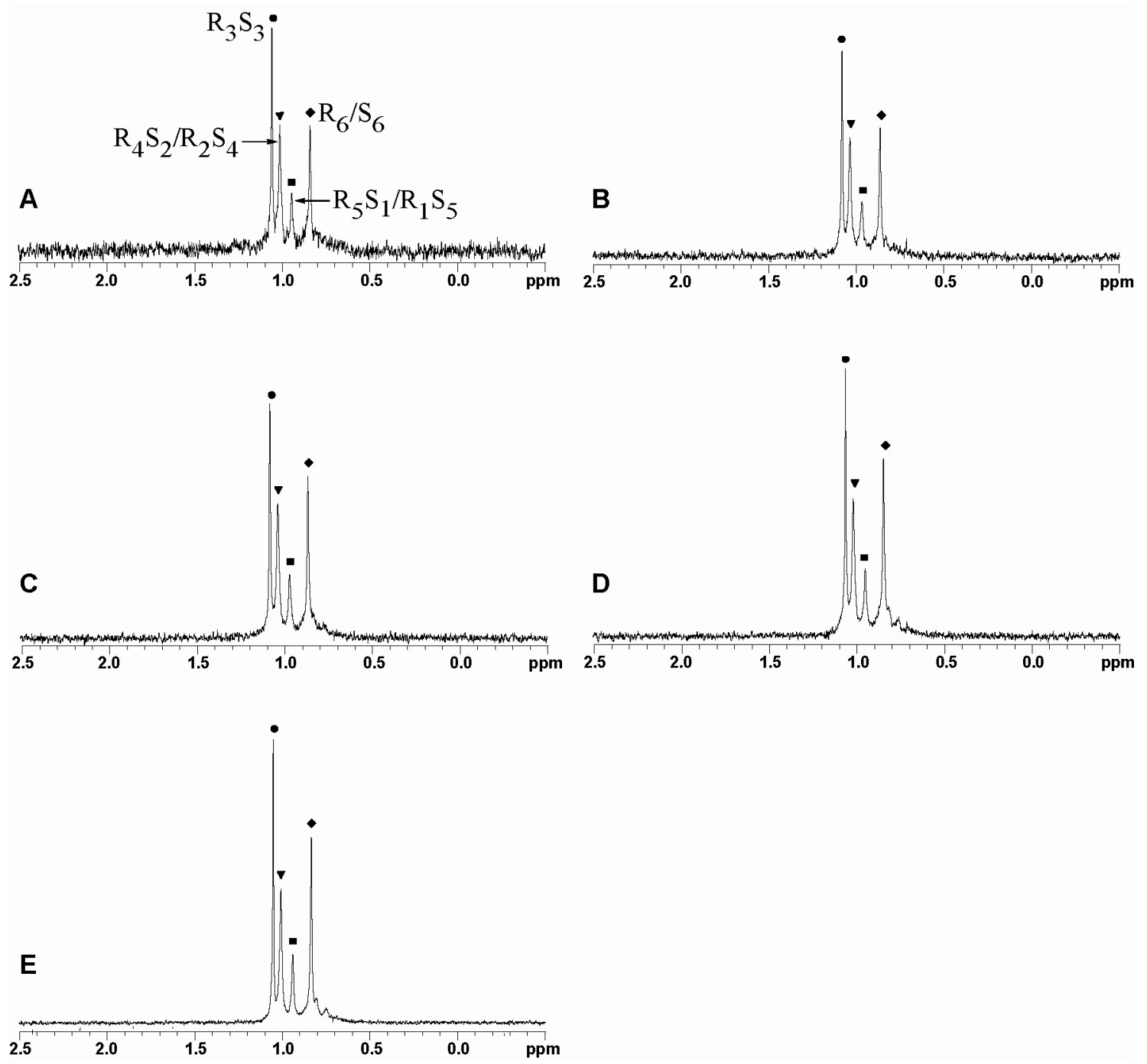

39. ${ }^{6} \mathrm{Li} \mathrm{NMR}$ spectra recorded on a mixture of $\left[{ }^{6} \mathrm{Li}\right](R)-9$ and $\left[{ }^{6} \mathrm{Li}\right] \mathrm{rac}-9(50 \%$ ee $)$ in $9.0 \mathrm{M}$ THF/toluene at $-50{ }^{\circ} \mathrm{C}$ at various enolate concentrations: (A) $0.04 \mathrm{M}$; (B) $0.10 \mathrm{M}$; (C) $0.15 \mathrm{M}$; (D) $0.25 \mathrm{M}$; (E) 0.40 M. $\mathbf{R}_{\mathbf{3}} \mathbf{S}_{\mathbf{3}}(\bullet) ; \mathbf{R}_{\mathbf{4}} \mathbf{S}_{2} / \mathbf{R}_{\mathbf{2}} \mathbf{S}_{\mathbf{4}}(\boldsymbol{\nabla}) ; \mathbf{R}_{\mathbf{5}} \mathbf{S}_{\mathbf{1}} / \mathbf{R}_{\mathbf{1}} \mathbf{S}_{\mathbf{5}}(\boldsymbol{\bullet}) ; \mathbf{R}_{\mathbf{6}} / \mathbf{S}_{\mathbf{6}}(\bullet) . \quad \mathbf{R}_{\mathbf{n}} \mathbf{S}_{\mathrm{N}-\mathrm{n}} / \mathbf{R}_{\mathrm{N}-\mathrm{n}} \mathbf{S}_{\mathbf{n}}$ and $\mathbf{R}_{\mathbf{N}} / \mathbf{S}_{\mathbf{N}}$ refer to pairs of spectroscopically indistinguishable enantiomers. 


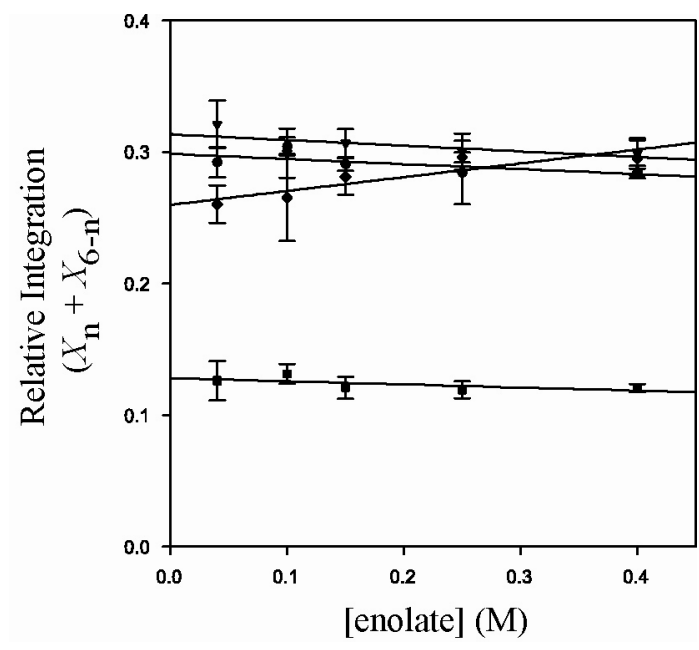

40. Plot of the mole fraction of the aggregate $\left(X_{n}+X_{6-n}\right)$ versus [enolate] for the spectra in Figure 39. For the case where $\mathrm{n}=3$, only $X_{3}$ is plotted. $\mathbf{R}_{\mathbf{3}} \mathbf{S}_{\mathbf{3}}(\bullet) ; \mathbf{R}_{\mathbf{4}} \mathbf{S}_{\mathbf{2}} / \mathbf{R}_{\mathbf{2}} \mathbf{S}_{\mathbf{4}}(\boldsymbol{\nabla}) ; \mathbf{R}_{\mathbf{5}} \mathbf{S}_{\mathbf{1}} / \mathbf{R}_{\mathbf{1}} \mathbf{S}_{\mathbf{5}}(\boldsymbol{\bullet}) ; \mathbf{R}_{\mathbf{6}} / \mathbf{S}_{\mathbf{6}}(\bullet)$. $\mathbf{R}_{n} \mathbf{S}_{\mathrm{N}-\mathrm{n}} / \mathbf{R}_{\mathrm{N}-\mathrm{n}} \mathbf{S}_{\mathbf{n}}$ and $\mathbf{R}_{\mathrm{N}} / \mathbf{S}_{\mathrm{N}}$ refer to pairs of spectroscopically indistinguishable enantiomers.

41. Table of data for the plot in Figure 40. $\left(\left[{ }^{6} \mathrm{Li}\right](R)-9\right.$ and $\left[{ }^{6} \mathrm{Li}\right] \mathrm{rac}-9(50 \%$ ee) $)$ in $9.0 \mathrm{M}$ $\mathrm{THF} /$ toluene at $-50{ }^{\circ} \mathrm{C}$.)

\begin{tabular}{ccccc}
\hline [enolate] (M) & $\mathbf{R}_{\mathbf{3}} \mathbf{S}_{\mathbf{3}}$ & $\mathbf{R}_{\mathbf{4}} \mathbf{S}_{\mathbf{2}} / \mathbf{R}_{\mathbf{2}} \mathbf{S}_{\mathbf{4}}$ & $\mathbf{R}_{\mathbf{5}} \mathbf{S}_{\mathbf{1}} / \mathbf{R}_{\mathbf{1}} \mathbf{S}_{\mathbf{5}}$ & $\mathbf{R}_{\mathbf{6}} / \mathbf{S}_{\mathbf{6}}$ \\
\hline 0.04 & $0.29 \pm 0.01$ & $0.32 \pm 0.02$ & $0.13 \pm 0.01$ & $0.26 \pm 0.01$ \\
0.10 & $0.30 \pm 0.01$ & $0.30 \pm 0.02$ & $0.13 \pm 0.01$ & $0.27 \pm 0.03$ \\
0.15 & $0.29 \pm 0.01$ & $0.31 \pm 0.01$ & $0.12 \pm 0.01$ & $0.28 \pm 0.01$ \\
0.25 & $0.28 \pm 0.02$ & $0.30 \pm 0.01$ & $0.12 \pm 0.01$ & $0.296 \pm 0.004$ \\
0.40 & $0.285 \pm 0.002$ & $0.30 \pm 0.01$ & $0.120 \pm 0.003$ & $0.30 \pm 0.02$ \\
\hline
\end{tabular}



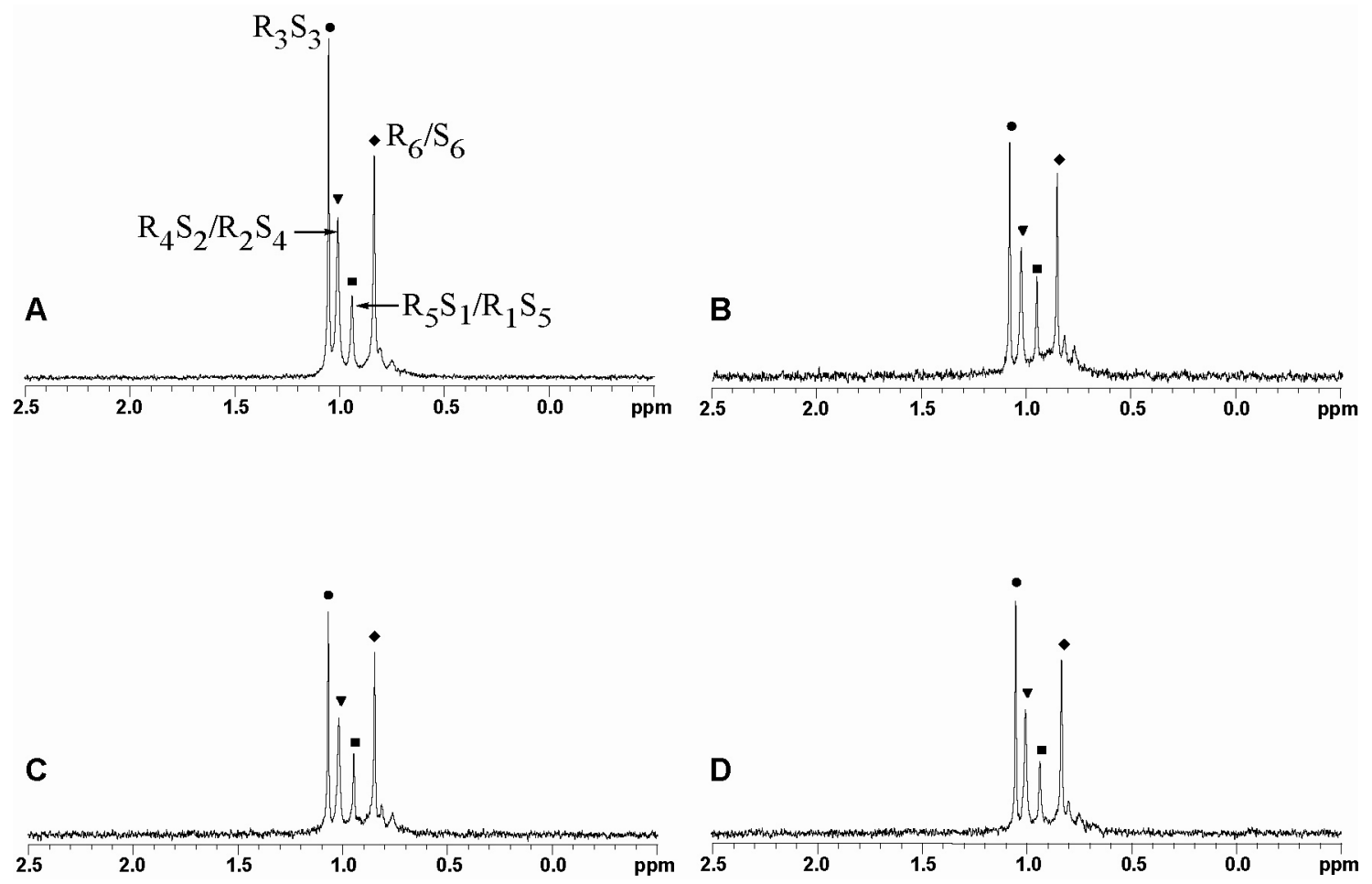

42. ${ }^{6} \mathrm{Li} \mathrm{NMR}$ spectra recorded on a mixture of $\left[{ }^{6} \mathrm{Li}\right](R)-9$ and $\left[{ }^{6} \mathrm{Li}\right] \mathrm{rac}-\mathbf{9}(50 \%$ ee $)$ in various THF/toluene mixtures at $-50{ }^{\circ} \mathrm{C}$ : (A) $2.0 \mathrm{M}$; (B) $4.0 \mathrm{M}$; (C) $6.0 \mathrm{M}$; (D) $8.0 \mathrm{M}$. $\mathbf{R}_{\mathbf{3}} \mathbf{S}_{\mathbf{3}}(\bullet$ ); $\mathbf{R}_{4} \mathbf{S}_{2} / \mathbf{R}_{2} \mathbf{S}_{4}(\boldsymbol{\nabla}) ; \mathbf{R}_{5} \mathbf{S}_{1} / \mathbf{R}_{1} \mathbf{S}_{5}(-) ; \mathbf{R}_{6} / \mathbf{S}_{6}(\bullet) . \mathbf{R}_{\mathbf{n}} \mathbf{S}_{\mathrm{N}-\mathrm{n}} / \mathbf{R}_{\mathrm{N}-\mathrm{n}} \mathbf{S}_{\mathbf{n}}$ and $\mathbf{R}_{\mathrm{N}} / \mathbf{S}_{\mathbf{N}}$ refer to pairs of spectroscopically indistinguishable enantiomers. 


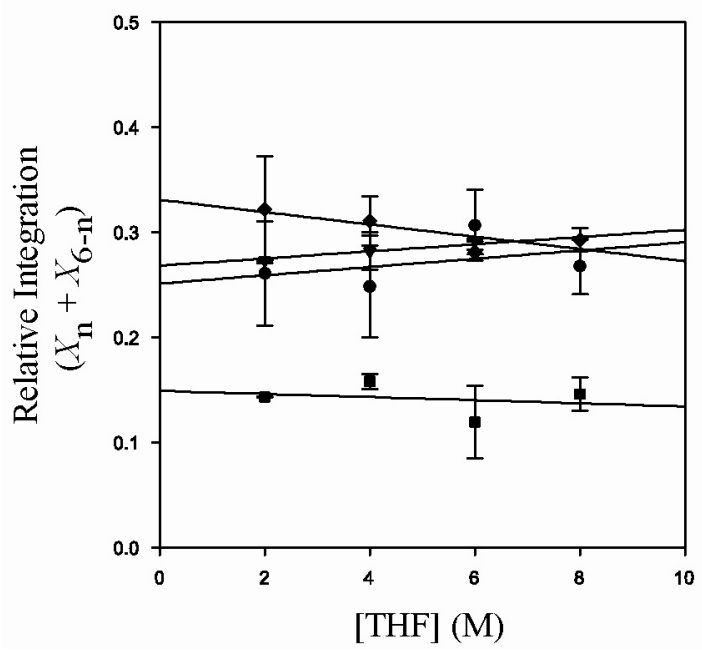

43. Plot of the mole fraction of the aggregate $\left(X_{n}+X_{6-n}\right)$ versus [THF] for the spectra in Figure 42. For the case where $n=3$, only $X_{3}$ is plotted. $\mathbf{R}_{\mathbf{3}} \mathbf{S}_{\mathbf{3}}(\bullet) ; \mathbf{R}_{\mathbf{4}} \mathbf{S}_{\mathbf{2}} / \mathbf{R}_{\mathbf{2}} \mathbf{S}_{\mathbf{4}}(\boldsymbol{v}) ; \mathbf{R}_{5} \mathbf{S}_{\mathbf{1}} / \mathbf{R}_{\mathbf{1}} \mathbf{S}_{\mathbf{5}}(\bullet) ; \mathbf{R}_{\mathbf{6}} / \mathbf{S}_{\mathbf{6}}(\bullet)$. $\mathbf{R}_{n} \mathbf{S}_{\mathbf{N}-\mathbf{n}} / \mathbf{R}_{\mathrm{N}-\mathrm{n}} \mathbf{S}_{\mathbf{n}}$ and $\mathbf{R}_{\mathrm{N}} / \mathbf{S}_{\mathbf{N}}$ refer to pairs of spectroscopically indistinguishable enantiomers.

44. Table of data for the plot in Figure 43. ([ $\left.{ }^{6} \mathrm{Li}\right](R)-9$ and $\left[{ }^{6} \mathrm{Li}\right] \mathrm{rac}-9(50 \%$ ee) in various THF concentrations (toluene co-solvent) at $-50{ }^{\circ} \mathrm{C}$.)

\begin{tabular}{ccccc}
\hline$[\mathrm{THF}](\mathrm{M})$ & $\mathbf{R}_{\mathbf{3}} \mathbf{S}_{\mathbf{3}}$ & $\mathbf{R}_{\mathbf{4}} \mathbf{S}_{\mathbf{2}} / \mathbf{R}_{\mathbf{2}} \mathbf{S}_{\mathbf{4}}$ & $\mathbf{R}_{\mathbf{5}} \mathbf{S}_{\mathbf{1}} / \mathbf{R}_{\mathbf{1}} \mathbf{S}_{\mathbf{5}}$ & $\mathbf{R}_{\mathbf{6}} / \mathbf{S}_{\mathbf{6}}$ \\
\hline 2.0 & $0.26 \pm 0.05$ & $0.274 \pm 0.001$ & $0.143 \pm 0.001$ & $0.32 \pm 0.05$ \\
4.0 & $0.25 \pm 0.05$ & $0.28 \pm 0.02$ & $0.16 \pm 0.01$ & $0.31 \pm 0.02$ \\
6.0 & $0.31 \pm 0.03$ & $0.293 \pm 0.001$ & $0.12 \pm 0.03$ & $0.281 \pm 0.002$ \\
8.0 & $0.27 \pm 0.03$ & $0.293 \pm 0.001$ & $0.15 \pm 0.02$ & $0.29 \pm 0.01$ \\
\hline
\end{tabular}



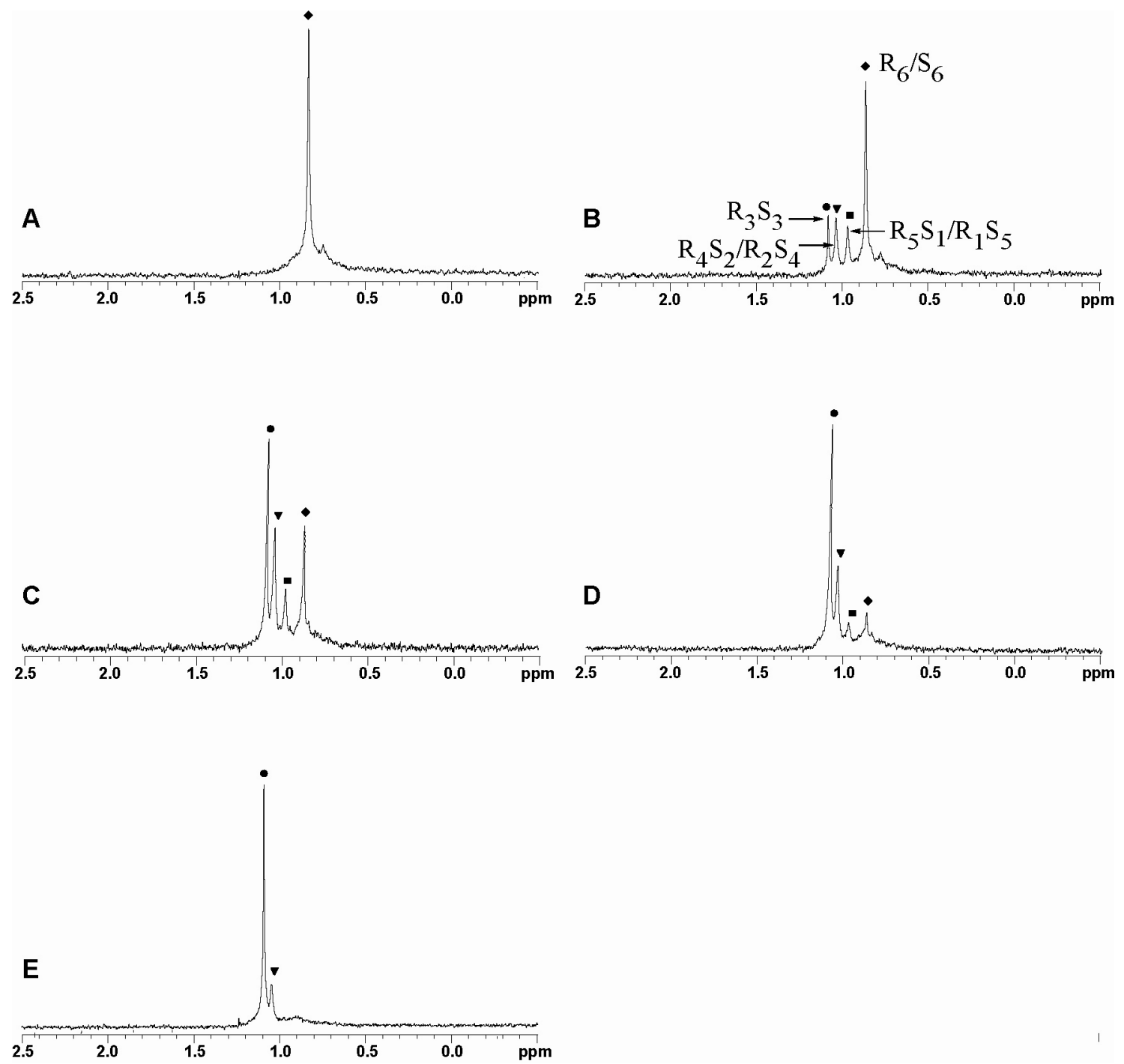

45. ${ }^{6} \mathrm{Li}$ NMR spectra recorded on mixtures of $\left[{ }^{6} \mathrm{Li}\right](S)-9$ and $\left[{ }^{6} \mathrm{Li}\right] \mathrm{rac}-9\left([\text { enolate }]_{\text {total }}=0.10 \mathrm{M}\right)$ at $-50{ }^{\circ} \mathrm{C}$ in $9.0 \mathrm{M} \mathrm{THF} /$ toluene: (A) $X_{R}=0.0$; (B) $X_{R}=0.15$; (C) $X_{R}=0.25$; (D) $X_{R}=0.35$; (E) $X_{R}=$ 0.45. $\mathbf{R}_{3} \mathbf{S}_{3}(\bullet) ; \mathbf{R}_{4} \mathbf{S}_{2} / \mathbf{R}_{2} \mathbf{S}_{4}(\bullet) ; \mathbf{R}_{5} \mathbf{S}_{1} / \mathbf{R}_{\mathbf{1}} \mathbf{S}_{5}(\bullet) ; \mathbf{R}_{6} / \mathbf{S}_{\mathbf{6}}(\bullet) . \mathbf{R}_{\mathbf{n}} \mathbf{S}_{\mathrm{N}-\mathrm{n}} / \mathbf{R}_{\mathrm{N}-\mathrm{n}} \mathbf{S}_{\mathbf{n}}$ and $\mathbf{R}_{\mathrm{N}} / \mathbf{S}_{\mathrm{N}}$ refer to pairs of spectroscopically indistinguishable enantiomers. 


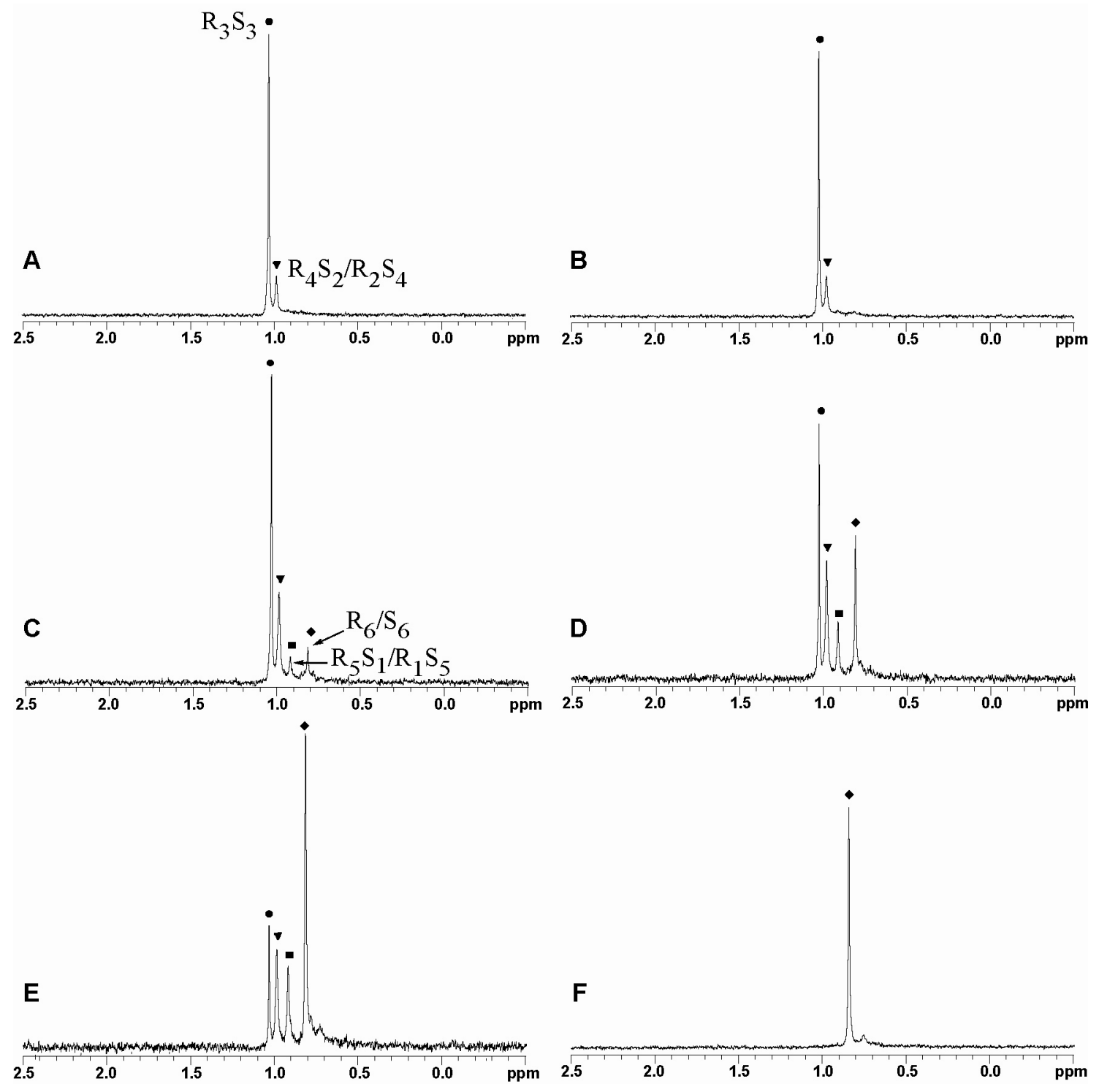

46. ${ }^{6} \mathrm{Li} \mathrm{NMR}$ spectra recorded on mixtures of $\left[{ }^{6} \mathrm{Li}\right](R)-9$ and $\left[{ }^{6} \mathrm{Li}\right] \mathrm{rac}-9\left([\text { enolate }]_{\text {total }}=0.10 \mathrm{M}\right)$ at $-50{ }^{\circ} \mathrm{C}$ in $9.0 \mathrm{M} \mathrm{THF} /$ toluene: (A) $X_{R}=0.50$; (B) $X_{R}=0.55$; (C) $X_{R}=0.65$; (D) $X_{R}=0.75$; (E) $X_{R}$ $=0.85 ;(\mathrm{F}) X_{R}=1.0 . \quad \mathbf{R}_{\mathbf{3}} \mathbf{S}_{\mathbf{3}}(\bullet) ; \mathbf{R}_{\mathbf{4}} \mathbf{S}_{\mathbf{2}} / \mathbf{R}_{\mathbf{2}} \mathbf{S}_{\mathbf{4}}(\boldsymbol{\bullet}) ; \mathbf{R}_{\mathbf{5}} \mathbf{S}_{\mathbf{1}} / \mathbf{R}_{\mathbf{1}} \mathbf{S}_{\mathbf{5}}(\bullet) ; \mathbf{R}_{\mathbf{6}} / \mathbf{S}_{\mathbf{6}}(\bullet) . \quad \mathbf{R}_{\mathbf{n}} \mathbf{S}_{\mathbf{N}-\mathbf{n}} / \mathbf{R}_{\mathbf{N}-\mathbf{n}} \mathbf{S}_{\mathbf{n}}$ and $\mathbf{R}_{\mathbf{N}} / \mathbf{S}_{\mathbf{N}}$ refer to pairs of spectroscopically indistinguishable enantiomers. 


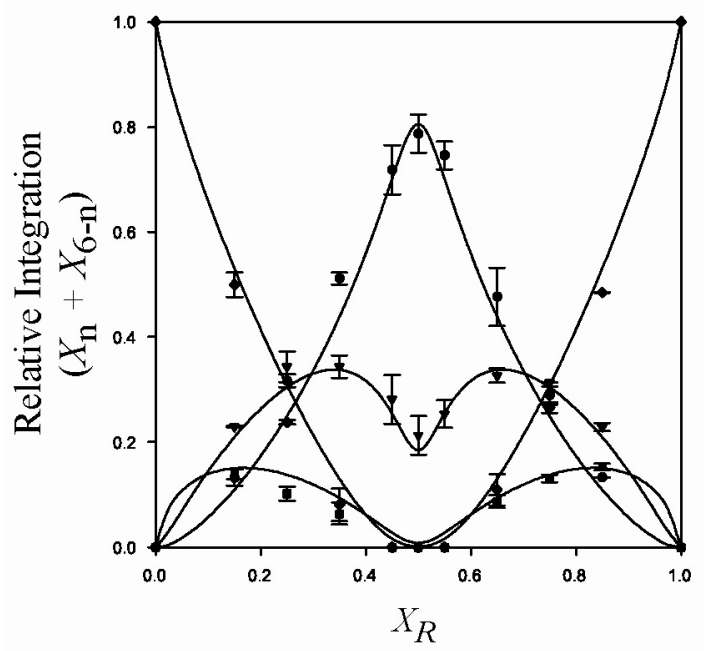

47. Plot of the mole fraction of the aggregate $\left(X_{\mathrm{n}}+X_{6-\mathrm{n}}\right)$ versus the mole fraction of $R\left(X_{R}\right)$ for the spectra in Figures 45 and 46. For the case where $n=3$, only $X_{3}$ is plotted. $\mathbf{R}_{\mathbf{3}} \mathbf{S}_{\mathbf{3}}(\bullet) ; \mathbf{R}_{\mathbf{4}} \mathbf{S}_{\mathbf{2}} / \mathbf{R}_{\mathbf{2}} \mathbf{S}_{\mathbf{4}}$ $(\mathbf{v}) ; \mathbf{R}_{5} \mathbf{S}_{\mathbf{1}} / \mathbf{R}_{\mathbf{1}} \mathbf{S}_{\mathbf{5}}(-) ; \mathbf{R}_{\mathbf{6}} / \mathbf{S}_{\mathbf{6}}(\bullet) . \mathbf{R}_{\mathbf{n}} \mathbf{S}_{\mathrm{N}-\mathrm{n}} / \mathbf{R}_{\mathrm{N}-\mathrm{n}} \mathbf{S}_{\mathbf{n}}$ and $\mathbf{R}_{\mathbf{N}} / \mathbf{S}_{\mathbf{N}}$ refer to pairs of spectroscopically indistinguishable enantiomers. See "Mathematical Derivations" section (pp S49-68) for details of the fit.

48. Table of data for the plot in Figure 47. (0.10 $\mathrm{M}$ [enolate $]_{\text {total }}$ at $-50{ }^{\circ} \mathrm{C}$ in $9.0 \mathrm{M} \mathrm{THF} /$ toluene.)

\begin{tabular}{ccccc}
\hline$X_{R}$ & $\mathbf{R}_{\mathbf{3}} \mathbf{S}_{\mathbf{3}}$ & $\mathbf{R}_{\mathbf{4}} \mathbf{S}_{\mathbf{2}} / \mathbf{R}_{\mathbf{2}} \mathbf{S}_{\mathbf{4}}$ & $\mathbf{R}_{\mathbf{5}} \mathbf{S}_{\mathbf{1}} / \mathbf{R}_{\mathbf{1}} \mathbf{S}_{\mathbf{5}}$ & $\mathbf{R}_{\mathbf{6}} / \mathbf{S}_{\mathbf{6}}$ \\
\hline 0.00 & $0.00 \pm 0.00$ & $0.00 \pm 0.00$ & $0.00 \pm 0.00$ & $1.00 \pm 0.00$ \\
0.15 & $0.13 \pm 0.02$ & $0.230 \pm 0.001$ & $0.14 \pm 0.01$ & $0.50 \pm 0.02$ \\
0.25 & $0.32 \pm 0.01$ & $0.34 \pm 0.03$ & $0.10 \pm 0.01$ & $0.238 \pm 0.003$ \\
0.35 & $0.51 \pm 0.01$ & $0.34 \pm 0.02$ & $0.06 \pm 0.02$ & $0.08 \pm 0.03$ \\
0.40 & $0.72 \pm 0.05$ & $0.28 \pm 0.05$ & $0.00 \pm 0.00$ & $0.00 \pm 0.00$ \\
0.50 & $0.79 \pm 0.04$ & $0.21 \pm 0.04$ & $0.00 \pm 0.00$ & $0.00 \pm 0.00$ \\
0.55 & $0.75 \pm 0.03$ & $0.25 \pm 0.03$ & $0.00 \pm 0.00$ & $0.00 \pm 0.00$ \\
0.65 & $0.48 \pm 0.06$ & $0.33 \pm 0.01$ & $0.09 \pm 0.01$ & $0.11 \pm 0.03$ \\
0.75 & $0.29 \pm 0.02$ & $0.314 \pm 0.001$ & $0.13 \pm 0.01$ & $0.27 \pm 0.01$ \\
0.85 & $0.133 \pm 0.001$ & $0.23 \pm 0.01$ & $0.15 \pm 0.01$ & $0.485 \pm 0.001$ \\
1.00 & $0.00 \pm 0.00$ & $0.00 \pm 0.00$ & $0.00 \pm 0.00$ & $1.00 \pm 0.00$ \\
\hline
\end{tabular}



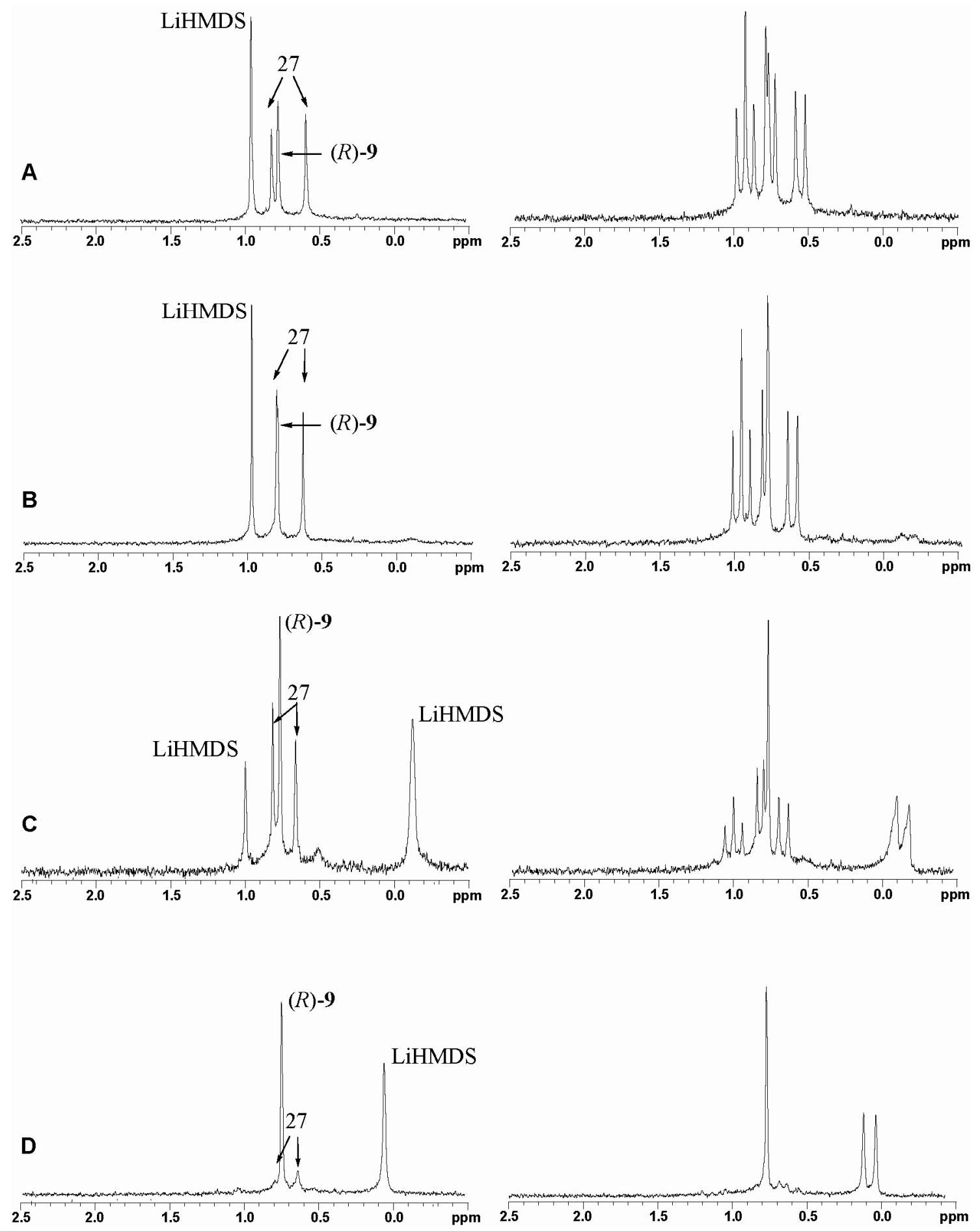

49. ${ }^{6} \mathrm{Li}\left\{{ }^{15} \mathrm{~N}\right\}$ and ${ }^{6} \mathrm{Li} \mathrm{NMR}$ spectra recorded on mixtures of $\left[{ }^{6} \mathrm{Li}\right](R)-9(0.07 \mathrm{M})$ and $\left[{ }^{6} \mathrm{Li}\right.$, $\left.{ }^{15} \mathrm{~N}\right] \mathrm{LiHMDS}(0.07 \mathrm{M})$ at $-90{ }^{\circ} \mathrm{C}$ in THF/toluene: (A) $[\mathrm{THF}]=0.6 \mathrm{M}$; (B) $[\mathrm{THF}]=3.1 \mathrm{M}$; (C) $[\mathrm{THF}]=6.2 \mathrm{M}$; (D) $[\mathrm{THF}]=12.3 \mathrm{M}$. 

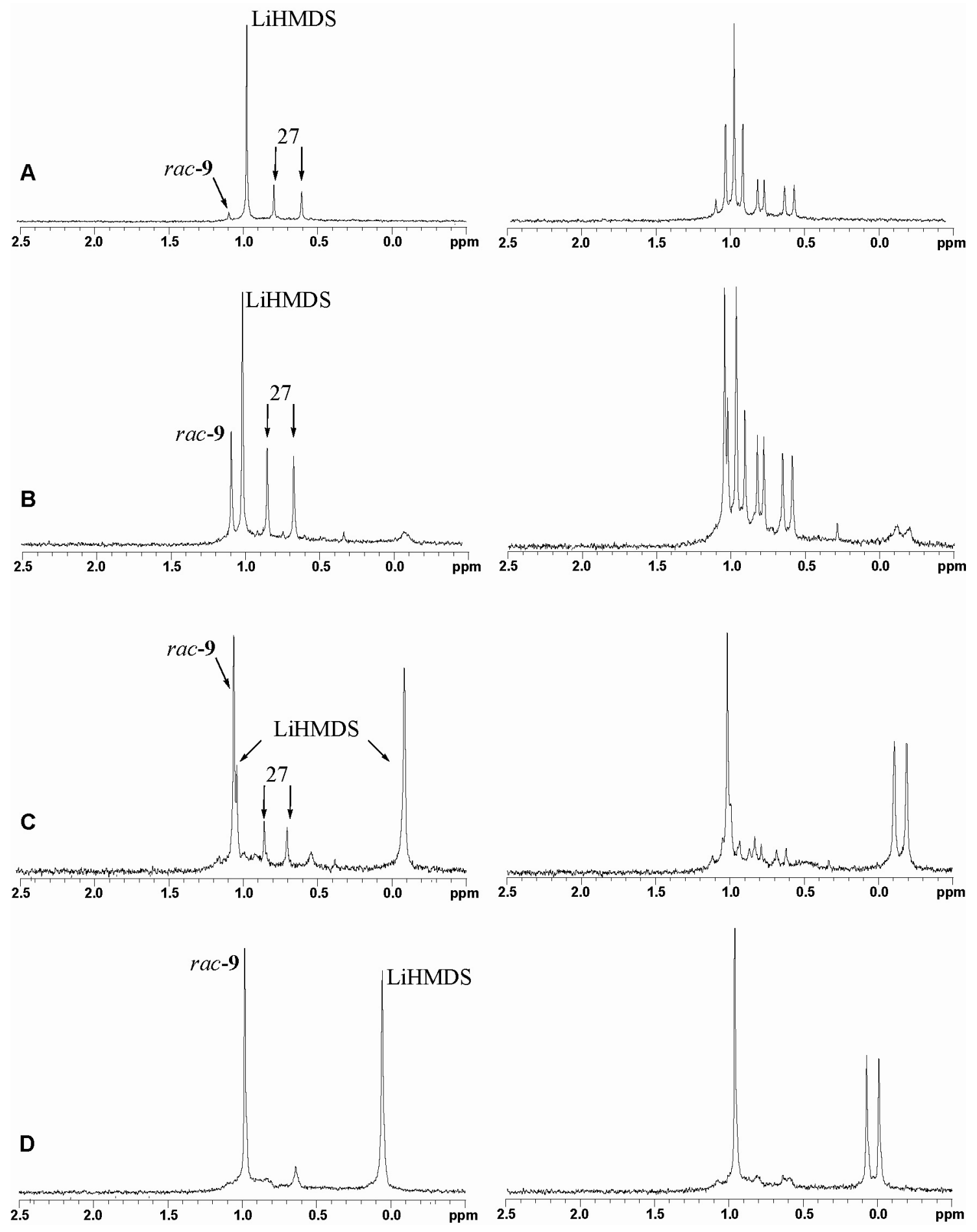

50. ${ }^{6} \mathrm{Li}\left\{{ }^{15} \mathrm{~N}\right\}$ and ${ }^{6} \mathrm{Li}$ NMR spectra recorded on mixtures of $\left[{ }^{6} \mathrm{Li}\right] \mathrm{rac}-9(0.07 \mathrm{M})$ and $\left[{ }^{6} \mathrm{Li}\right.$, $\left.{ }^{15} \mathrm{~N}\right]$ LiHMDS (0.07 M) at $-90{ }^{\circ} \mathrm{C}$ in THF/toluene: (A) $[\mathrm{THF}]=0.6 \mathrm{M}$; (B) $[\mathrm{THF}]=3.1 \mathrm{M}$; (C) $[\mathrm{THF}]=6.2 \mathrm{M}$; (D) $[\mathrm{THF}]=12.3 \mathrm{M}$. 

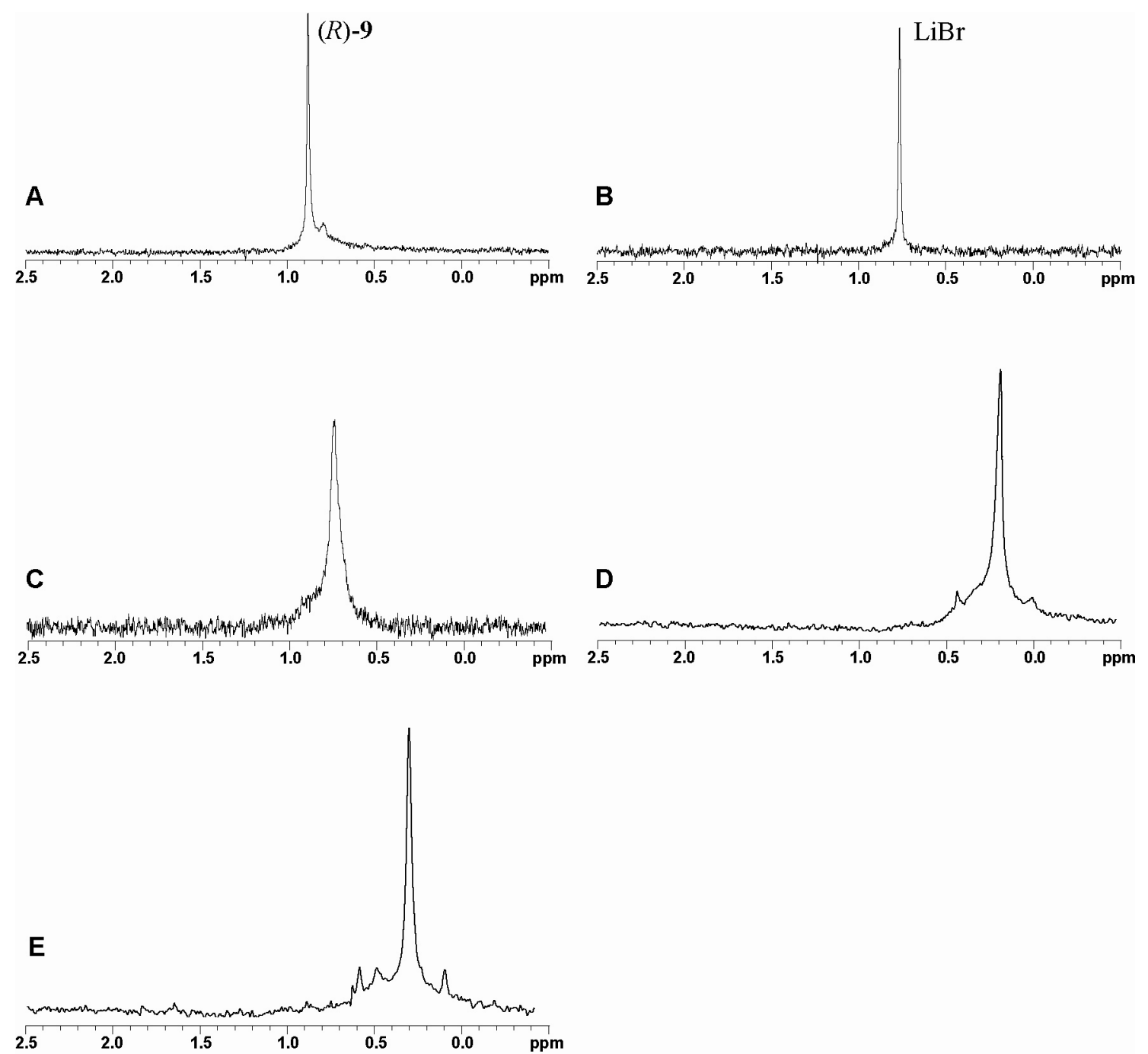

51. ${ }^{6} \mathrm{Li} \mathrm{NMR}$ spectra recorded on mixtures of $\left[{ }^{6} \mathrm{Li}\right](R)-9$ and $\left[{ }^{6} \mathrm{Li}\right] \mathrm{LiBr}$ in $9.0 \mathrm{M} \mathrm{THF} /$ toluene at various temperatures: $(\mathrm{A})(R)-9(0.10 \mathrm{M}),-50{ }^{\circ} \mathrm{C}$; (B) $\mathrm{LiBr}(0.10 \mathrm{M}),-50{ }^{\circ} \mathrm{C} ;(\mathrm{C})(R)-9 / \mathrm{LiBr}$ $(0.10 \mathrm{M} / 0.10 \mathrm{M}),-50{ }^{\circ} \mathrm{C}$; (D) $(R)-9 / \operatorname{LiBr}(0.10 \mathrm{M} / 0.10 \mathrm{M}),-70{ }^{\circ} \mathrm{C} ;(\mathrm{E})(R)-9 / \operatorname{LiBr}(0.10 \mathrm{M} / 0.10$ $\mathrm{M}),-90{ }^{\circ} \mathrm{C}$. 

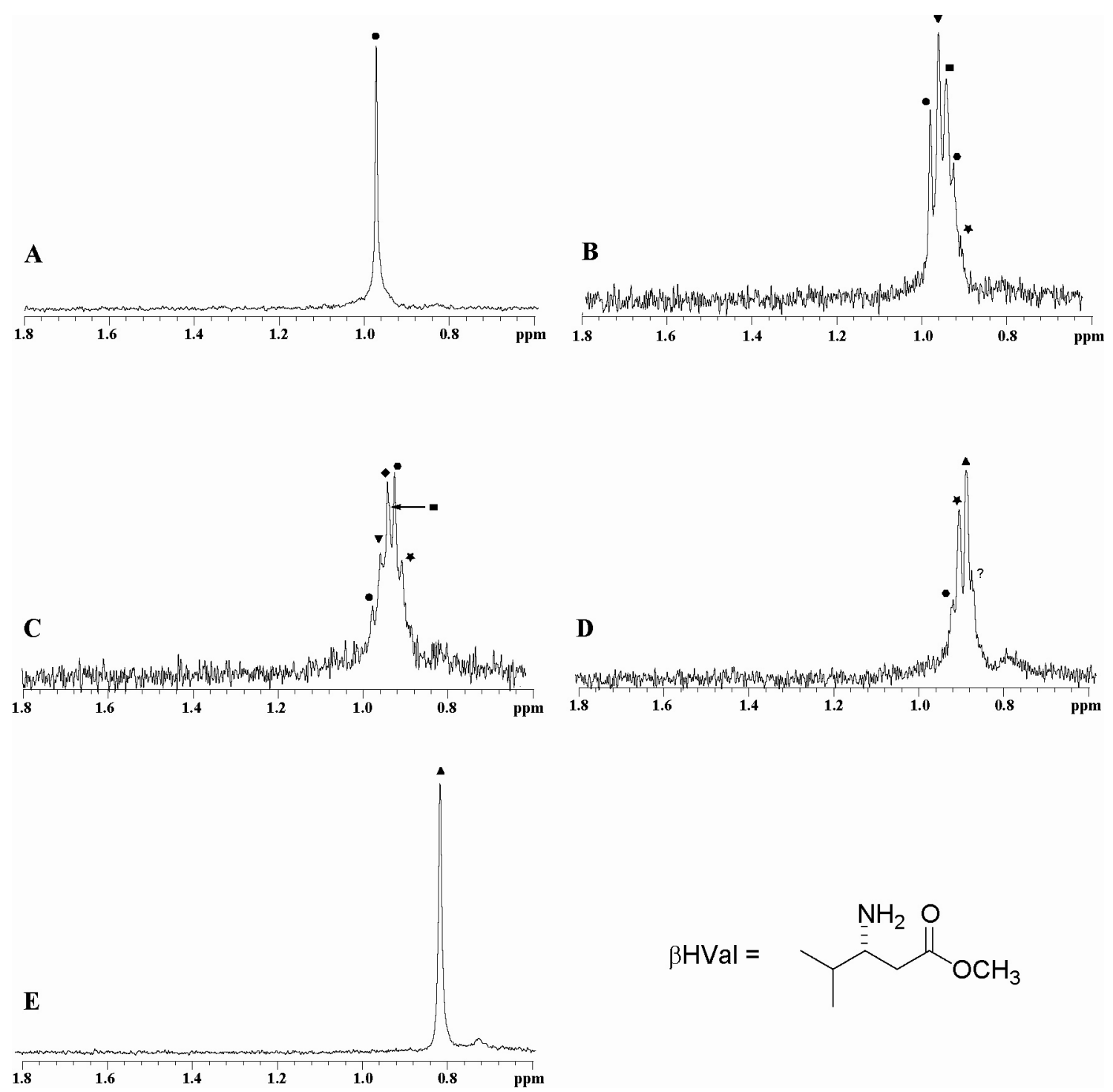

52. ${ }^{6} \mathrm{Li}$ NMR spectra recorded on mixtures of $\left[{ }^{6} \mathrm{Li}\right](R)-9$ and $\left[{ }^{6} \mathrm{Li}\right] \beta \mathrm{HVal}$ in $9.8 \mathrm{M}$

THF/cyclopentane at $-50{ }^{\circ} \mathrm{C}$ : (A) $(R)-\mathbf{9}(0.10 \mathrm{M})$; (B) $(R)-9 / \beta H V a l(0.075 \mathrm{M} / 0.025 \mathrm{M})$; (C) $(R)-$

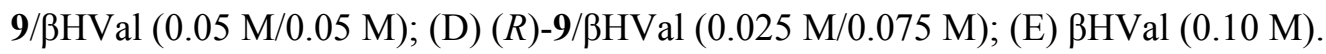




\section{MNDO Computations}

MNDO computations were performed using WinMOPAC version 2.0 by Fujitsu ${ }^{\circledR}$ Limited.

53. Relative heats of formation ( $\mathrm{kcal} / \mathrm{mol})$.

Homochiral

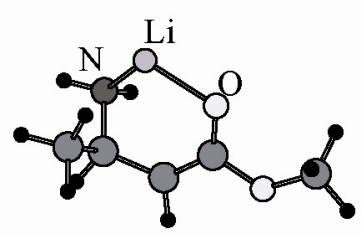

$\mathrm{R}_{1}$

$(6 \times-115.5)$

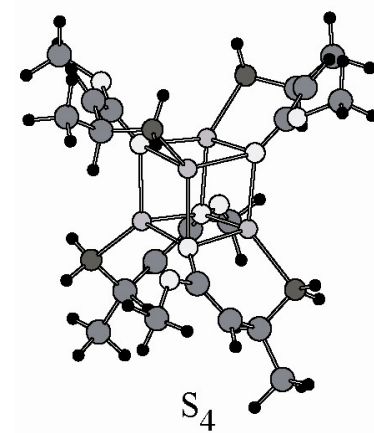

$(3 / 2 \times-511.8)$
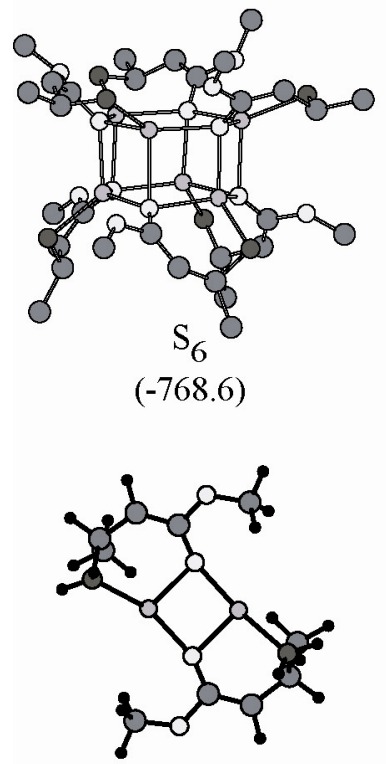

$\mathrm{R}_{2}$

$(3 \times-266.1)$
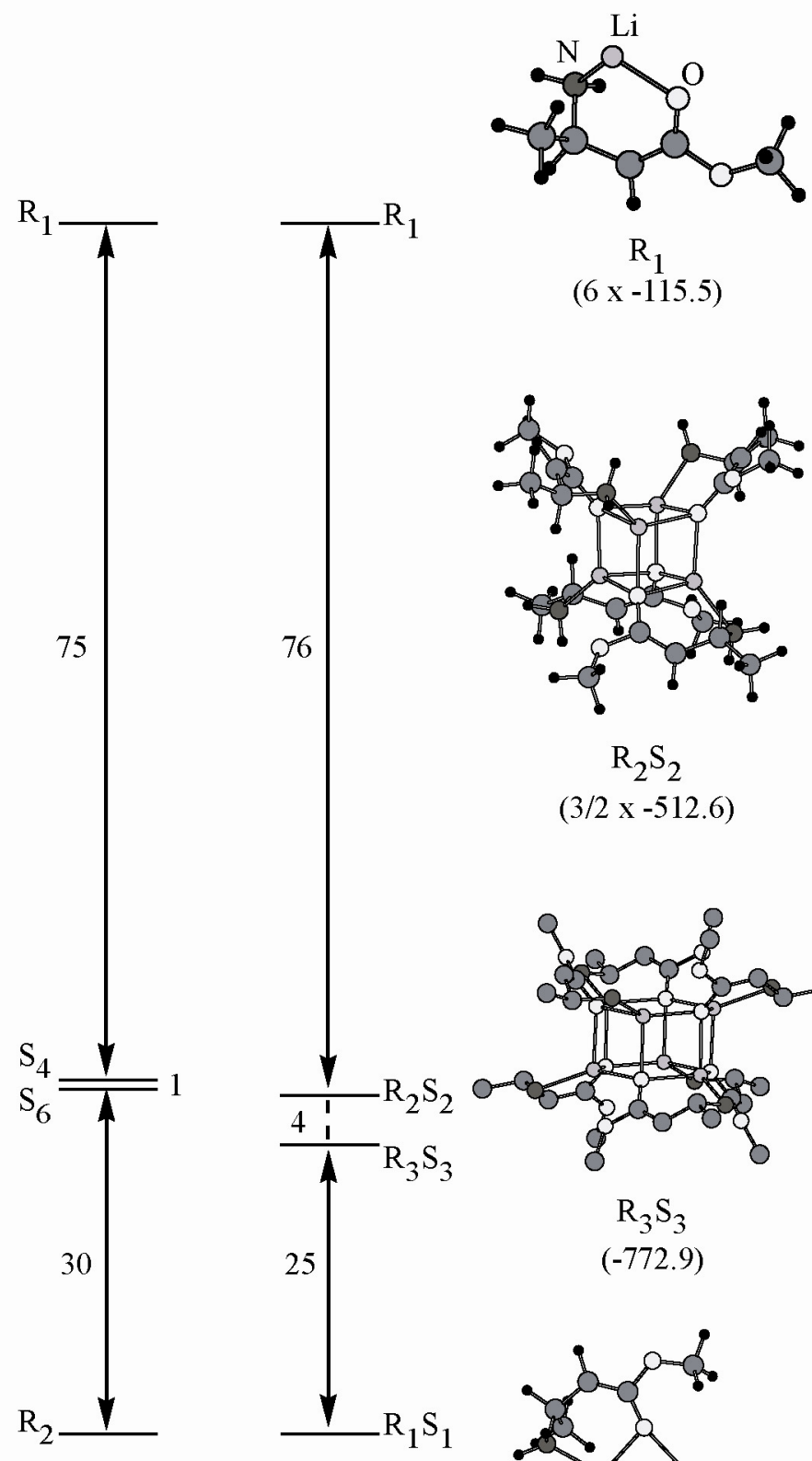

$\mathrm{R}_{1}$

$(6 \times-115.5)$

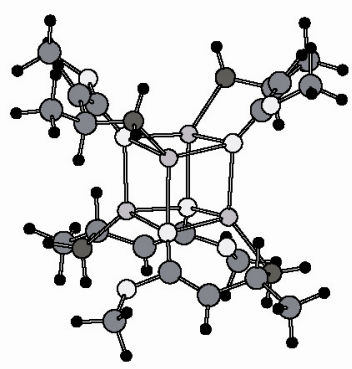

$\mathrm{R}_{2} \mathrm{~S}_{2}$

$(3 / 2 \times-512.6)$

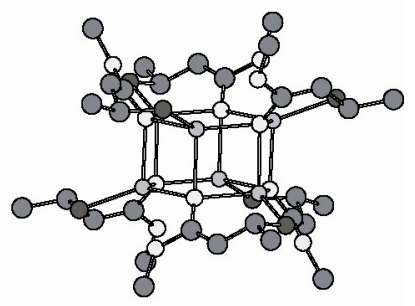

$\mathrm{R}_{3} \mathrm{~S}_{3}$

$(-772.9)$

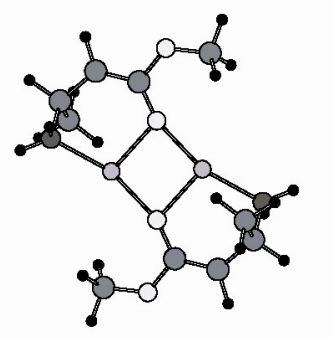

$\mathrm{R}_{1} \mathrm{~S}_{1}$

$(3 \times-266.1)$ 
54. Effect of symmetry on heats of formation for hexamers ( $\mathrm{kcal} / \mathrm{mol})$.

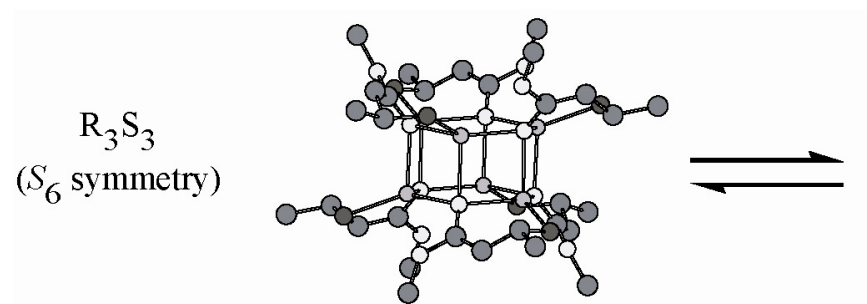

$(-772.9)$

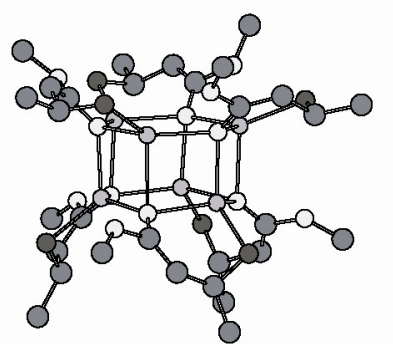

$(-768.6)$

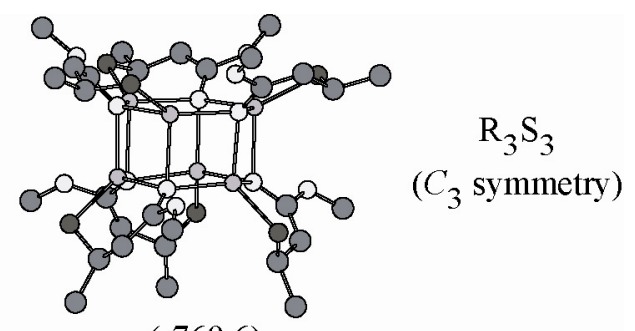

$(-769.6)$

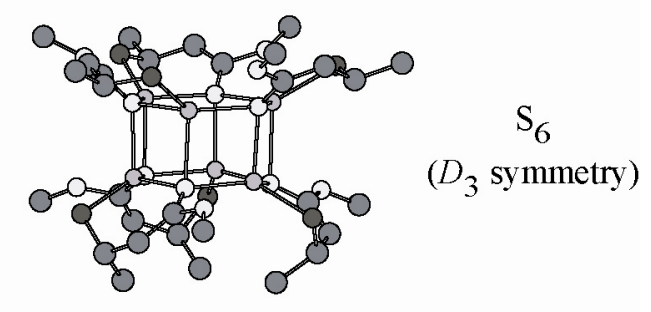

$(-765.5)$

55. Thermoneutral opening of the $R_{3} S_{3}$ hexamer to the ladder (kcal/mol).

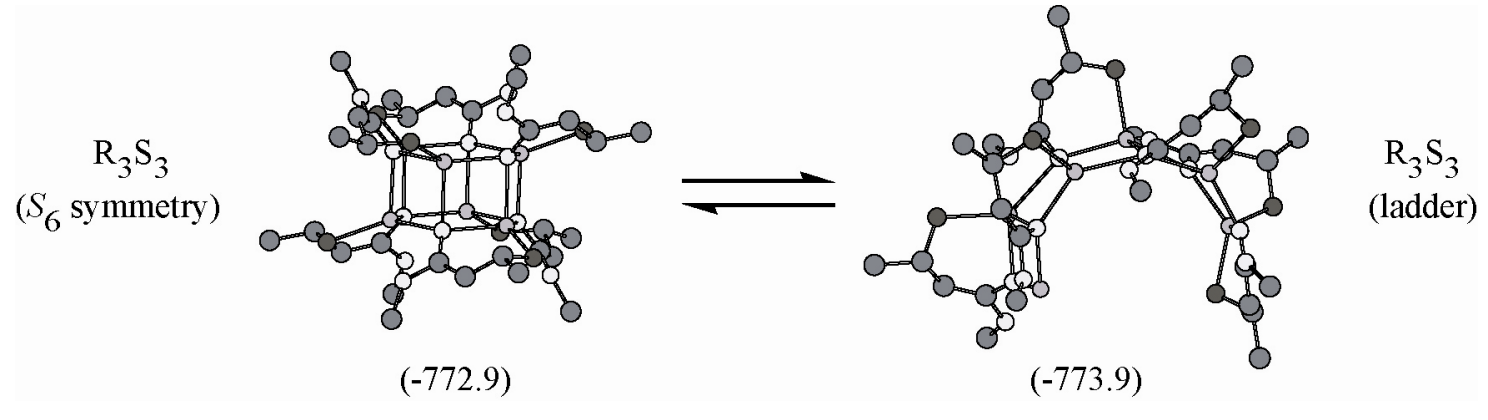


56. Transition structures for anti alkylation of the $\mathrm{R}_{3} \mathrm{~S}_{3}$ ladder with $\mathrm{CH}_{3} \mathrm{Br}(\mathrm{kcal} / \mathrm{mol})$.

Transition structures were verified by a single negative frequency at approximately $-700 \mathrm{~cm}^{-1}$ which corresponding to the $\mathrm{C}-\mathrm{C}-\mathrm{Br}$ stretch.

alkylation at doubly-chelated end

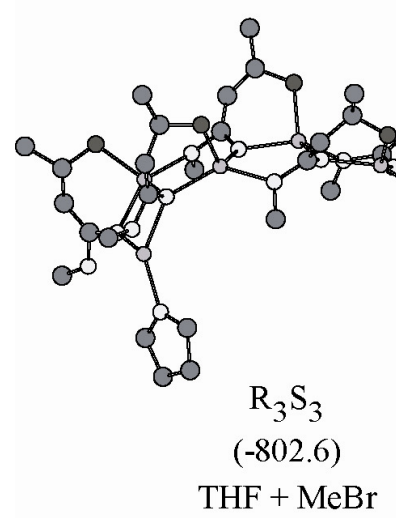

alkylation at mono-chelated end

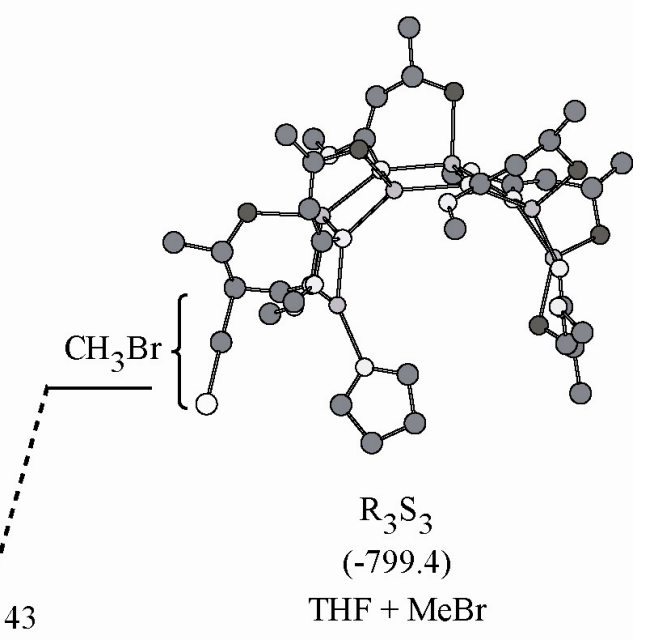

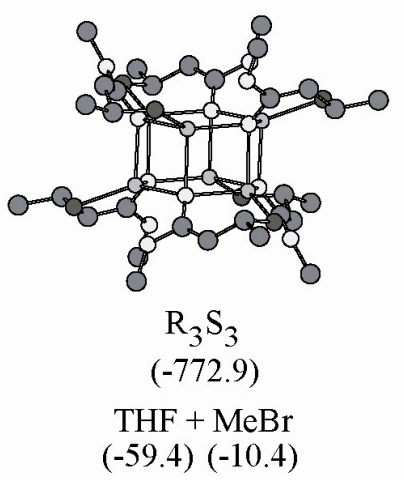


57. Transition structures for anti alkylation via a monomer with $\mathrm{CH}_{3} \mathrm{Br}$ ( $\left.\mathrm{kcal} / \mathrm{mol}\right)$.

Transition structures were verified by a single negative frequency at approximately $-700 \mathrm{~cm}^{-1}$ which corresponding to the $\mathrm{C}-\mathrm{C}-\mathrm{Br}$ stretch.

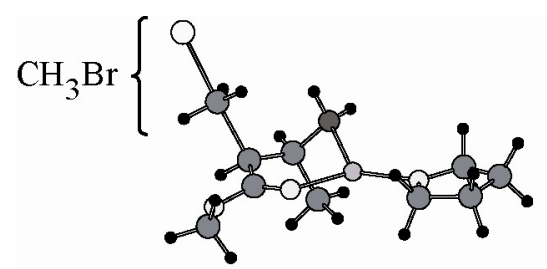

$(-148.1)$
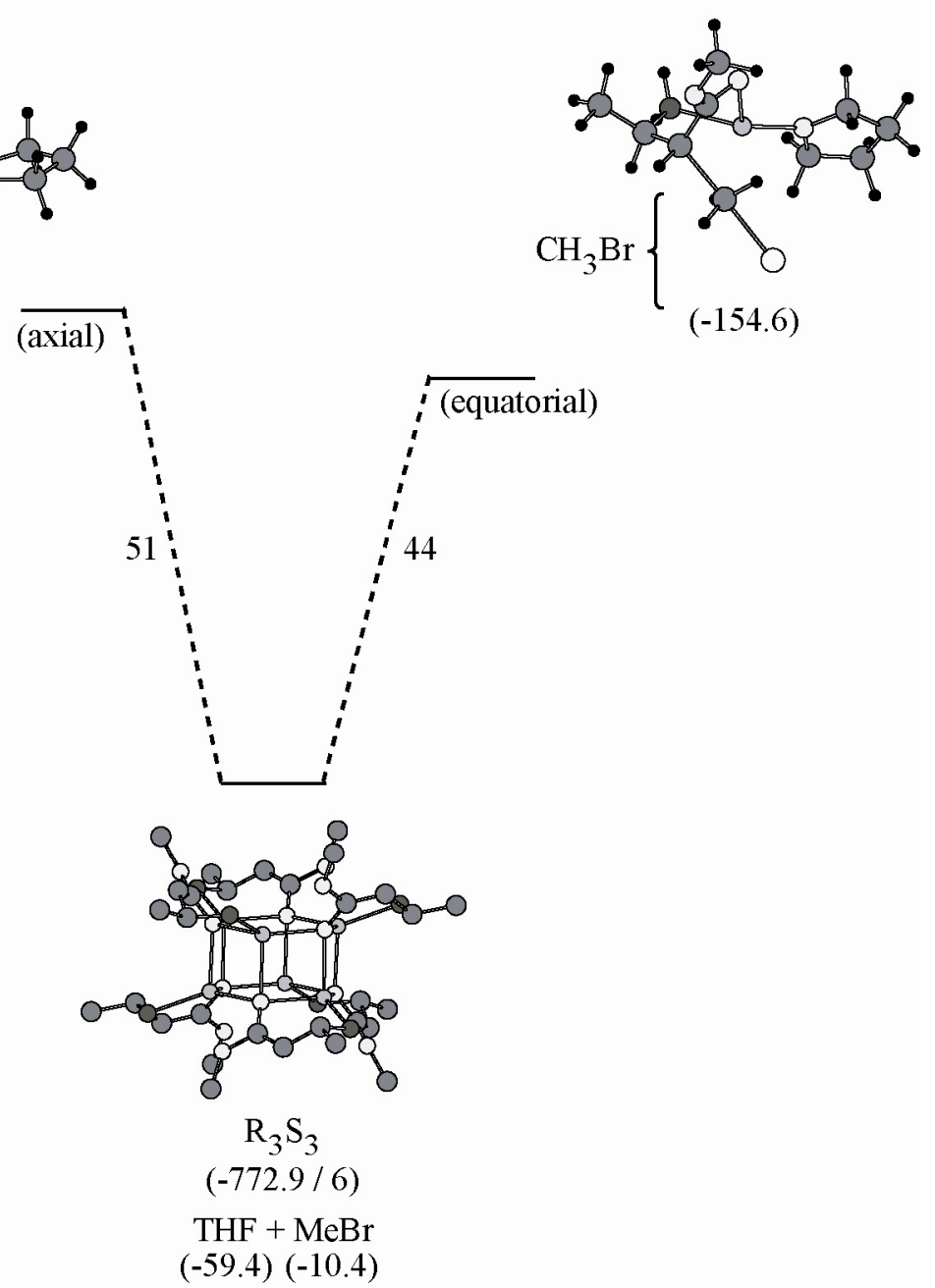
58. Transition structures for anti alkylation via a doubly-chelated dimer $\left(\mathrm{R}_{1} \mathrm{~S}_{1}\right)$ with $\mathrm{CH}_{3} \mathrm{Br}$ (kcal/mol). Transition structures were verified by a single negative frequency at approximately $700 \mathrm{~cm}^{-1}$ which corresponding to the C-C-Br stretch.

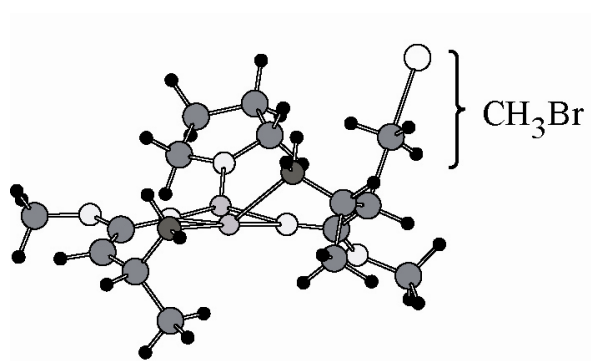

$(-281.5)$

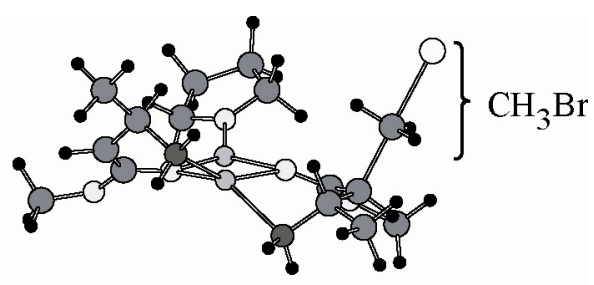

$(-278.2)$

(diaxial) (diequatorial)

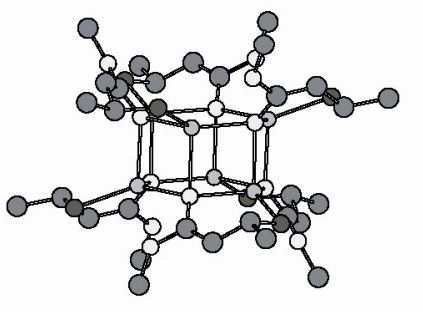

$$
\mathrm{R}_{3} \mathrm{~S}_{3}
$$

(-772.9/3)

$\mathrm{THF}+\mathrm{MeBr}$

$(-59.4)(-10.4)$ 


\section{Mathematical Derivations}

59. Introduction to the hexamer fitting protocol. We consider a situation where the two enantiomers, $(R)-\mathbf{9}$ and $(S)-9$, assemble in solution to form hexamers $(\mathrm{N}=6)$. For an individual hexamer, each of the six positions in the assembly can be occupied by an $(R)-\mathbf{9}$ or an $(S)-9$ (hereafter denoted as R and S, respectively). One way to describe a hexamer is by listing the occupant of each position - RSSRSR, RRRRRR, or RRSSRS for example. Rather than consider the concentration of each permutation, $\mathrm{P}$, we can group them according to the number of $\mathrm{R}$ subunits in the hexamer, $n_{P}$. The concentration, $\left[R_{n} S_{N-n}\right]$, of states for which $n_{P}=n$ is given by the Boltzmann distribution. It will depend on

1. Multiplicity $\left(M_{n}\right)$ : The number of permutations of $P$ for which $n_{P}=n$. By example, RSRSRS and SRRSSR are just two of 20 permutations with $n_{P}=3$.

2. Free Energy $\left(g_{\mathrm{P}}\right)$ : Each permutation may have a different energy of assembly/association. For example, RRRSSS may be a much less stable permutation than RSRSRS.

3. Chemical Potential $\left(\mu_{\mathrm{R}}\right.$ and $\left.\mu_{\mathrm{S}}\right)$ : The total amount of $\mathrm{R},\left[\mathrm{R}_{\text {total }}\right]$, and $\mathrm{S}$, $\left[\mathrm{S}_{\text {total }}\right]$, will set the chemical potentials and shift the likelihood of various states. If $\left[\mathrm{R}_{\text {total }}\right]>>\left[\mathrm{S}_{\text {total }}\right]$, for instance, then the $\left[\mathrm{R}_{1} \mathrm{~S}_{5}\right]$ will be much less likely than $\left[\mathrm{R}_{5} \mathrm{~S}_{1}\right]$.

In the experiment, the independent variable is the mole fraction of subunits of $\mathrm{R}, X_{R}$, and the dependent variables are linear combinations of the mole fraction of $\left[\mathrm{R}_{n} \mathrm{~S}_{\mathrm{N}-\mathrm{n}}\right], X_{\mathrm{n}}$. Thus, we wish to predict $X_{\mathrm{n}}$ as a function of $X_{R}$ for a given model.

In Section 60 we use the Boltzmann distribution to give the value of $\left[\mathrm{R}_{\mathrm{n}} \mathrm{S}_{\mathrm{N}-\mathrm{n}}\right]$ in terms of free energies, multiplicity and chemical potential. In Section 61 we give an explicit form for the multiplicity. The relationship between chemical potentials and [ $\left.\mathrm{R}_{\text {total }}\right]$, [ $\left.\mathrm{S}_{\text {total }}\right]$ (or $X_{R}$ and $X_{\mathrm{S}}$ ) is derived in Section 62.

Section 63 considers the case where the free energies of assembly for all the permutations are equal (statistical case) for which a simple analytic result is possible. As there are no model 
parameters in the statistical case, the data either fits the model or the statistical assumption is invalid.

The general case does not have a simple analytic solution. A parametric approach is described in Section 64. This numeric method allows one to compare the experimental and predicted populations, $X_{\mathrm{n}}$, for a given set of free energies. We obtain the residual error after an iterative optimization of the free energies to fit the data, thus giving a measure of the model's validity. Section 65 describes the implementation of this approach. Section 66 relates free energies to equilibrium constants within the system. Section 67 shows a fit of the experimental data to a heptamer model. Sections 68 and 69 give the equations and sample plots for a model with tetramers and a dimer-tetramer mixture, respectively. 
60. Boltzmann distribution. Consider a given permutation, $P$, with $n_{p}$ subunits of type $R$ and $\mathrm{N}-\mathrm{n}_{\mathrm{p}}$ subunits of type $\mathrm{S}$. The Boltzmann distribution gives its equilibrium concentration as

$$
[P]=C \times \exp \left(\frac{-g_{p}+n_{P} \mu_{R}+\left(N-n_{P}\right) \mu_{S}}{k T}\right)
$$

where $C$ is a constant, $g_{P}$ is the free energy of assembly of $P, \mu_{R}$ is the chemical potential of $R$ and $\mu_{\mathrm{S}}$ is the chemical potential of S (Widom, B. Statistical Mechanics: A Concise Introduction for Chemists; Cambridge University Press: New York, 2002). Within the experiment, all states for which $n_{P}=n$ are indistinguishable. The concentration of permutations for which $n_{p}=n$ is given by

$$
\begin{aligned}
{\left[R_{n} S_{N-n}\right] } & =\sum_{P ; n_{P}=n}[P]=C \times \exp \left(\frac{n \mu_{R}+(N-n) \mu_{S}}{k T}\right) \times \sum_{P ; n_{P}=n} \exp \left(\frac{-g_{P}}{k T}\right) \\
& =C \times \exp \left(\frac{n \mu_{R}+(N-n) \mu_{S}}{k T}\right) \times M_{n} \times\left\langle\exp \left(\frac{-g_{P}}{k T}\right)\right\rangle_{P ; n_{p}=n}
\end{aligned}
$$

where $M_{n}$ is the multiplicity (number of permutations $P$ where $n_{P}=n$ ) and the average of free energy is taken over all states for which $n_{P}=n$. It is helpful to define some "effective" variables

$$
r=\exp \left(\frac{\mu_{R}}{k T}\right) \quad s=\exp \left(\frac{\mu_{S}}{k T}\right) \quad \phi_{n}=\left\langle\exp \left(\frac{-g_{P}}{k T}\right)\right\rangle_{P ; n_{P}=n}
$$

Substituting these into the above expression gives

$$
\left[R_{n} S_{N-n}\right]=C \times M_{n} \times \phi_{n} \times r^{n} \times s^{N-n}
$$

Increasing the chemical potential of $\mathrm{R}$ increases the value of " $r$ " and favors the $\left[\mathrm{R}_{6} \mathrm{~S}_{0}\right]$, $\left[\mathrm{R}_{5} \mathrm{~S}_{1}\right]$, etc. states. Increasing the chemical potential of $\mathrm{S}$ increases the value of " $s$ " which then favors $\left[\mathrm{R}_{0} \mathrm{~S}_{6}\right],\left[\mathrm{R}_{1} \mathrm{~S}_{5}\right]$, etc.

$\varphi_{n}$ describes the mean free energy of permutations in $\left[R_{n} S_{N-n}\right]$. Increasing $\varphi_{n}$ favors $\left[R_{n} S_{N-n}\right]$ as would be expected if those states have a low free energy. Not all values of $\varphi_{\mathrm{n}}$ are independent. 
The free energy of a permutation, P, and the free energy of one in which R and S have been exchanged are the same because the aggregates are enantiomers. This has the important consequence that

$$
\phi_{n}=\phi_{N-n}
$$

Furthermore, free energies can only be measured relative to the free energy of a reference state. For example, if free energies are measured relative to that of $\left[\mathrm{R}_{6} \mathrm{~S}_{0}\right]$ then $\varphi_{0}=\varphi_{6}=1$. When $\mathrm{N}$ is even, $N / 2$ of the values of $\varphi_{n}$ are independent. For example, when $N=6, \varphi_{1}, \varphi_{2}$, and $\varphi_{3}$ are independent of each other. 
61. Multiplicity. The value of $M_{n}$ can be directly obtained by an exhaustive grouping of all hexamer permutations.

\begin{tabular}{|c|c|c|c|}
\hline Species & $\mathrm{n}$ & $\begin{array}{l}\mathrm{M}_{\mathrm{n}}-\text { Number of } \\
\text { permutations }\end{array}$ & Permutations \\
\hline $\mathrm{R}_{0} \mathrm{~S}_{6}$ & 0 & 1 & SSSSSS \\
\hline $\mathrm{R}_{1} \mathrm{~S}_{5}$ & 1 & 6 & RSSSSS, SRSSSS, SSRSSS, SSSRSS, SSSSRS, SSSSSR \\
\hline $\mathrm{R}_{2} \mathrm{~S}_{4}$ & 2 & 15 & $\begin{array}{l}\text { RRSSSS, RSRSSS, RSSRSS, RSSSRS, RSSSSR, SRRSSS, } \\
\text { SRSRSS, SRSSRS, SRSSSR, SSRRSS, SSRSRS, SSRSSR, } \\
\text { SSSRRS, SSSRSR, SSSSRR }\end{array}$ \\
\hline $\mathrm{R}_{3} \mathrm{~S}_{3}$ & 3 & 20 & $\begin{array}{l}\text { RRRSSS, RRSRSS, RRSSRS, RRSSSR, RSRRSS, RSRSRS, } \\
\text { RSRSSR, RSSRRS, RSSRSR, RSSSRR, + } 10 \text { other states } \\
\text { with R and S switched }\end{array}$ \\
\hline $\mathrm{R}_{4} \mathrm{~S}_{2}$ & 4 & 15 & $\begin{array}{l}\text { SSRRRR, SRSRRR, SRRSRR, SRRRSR, SRRRRS, } \\
\text { RSSRRR, RSRSRR, RSRRSR, RSRRRS, RRSSRR, } \\
\text { RRSRSR, RRSRRS, RRRSSR, RRRSRS, RRRRSS }\end{array}$ \\
\hline $\mathrm{R}_{5} \mathrm{~S}_{1}$ & 5 & 6 & $\begin{array}{c}\text { SRRRRR, RSRRRR, RRSRRR, RRRSRR, RRRRSR, } \\
\text { RRRRRS }\end{array}$ \\
\hline $\mathrm{R}_{6} \mathrm{~S}_{0}$ & 6 & 1 & RRRRRR \\
\hline
\end{tabular}

Alternatively, one can use Pascal's triangle or the binomial theorem to achieve the general result

$$
\text { Multiplicity }=\mathrm{M}_{\mathrm{n}}=\frac{N !}{(N-n) ! \times n !} .
$$


62. Chemical potential. The experimental variables are the mole fractions of $\left[\mathrm{R}_{\mathrm{n}} \mathrm{S}_{\mathrm{N}-\mathrm{n}}\right], X_{\mathrm{n}}$, and the mole fraction of R, $X_{R}$. Their relationships to $\mathrm{C}, r$ and $s$ are described below.

Using eq 1 to compute $\left[\mathrm{R}_{\mathrm{n}} \mathrm{S}_{\mathrm{N}-\mathrm{n}}\right]$, the mole fraction $X_{\mathrm{n}}$ is given by

$$
\begin{aligned}
X_{n} & =\frac{\left[R_{n} S_{N-n}\right]}{\sum_{j=0}^{N}\left[R_{j} S_{N-j}\right]}=\frac{C \times M_{n} \times \phi_{n} \times r^{n} \times s^{N-n}}{\sum_{j=0}^{N} C \times M_{j} \times \phi_{j} \times r^{j} \times s^{N-j}}=\frac{M_{n} \times \phi_{n} \times\left(\frac{r}{s}\right)^{n}}{\sum_{j=0}^{N} M_{j} \times \phi_{j} \times\left(\frac{r}{s}\right)^{j}} \\
= & \frac{M_{n} \times \phi_{n} \times \exp \left(\frac{n \times\left(\mu_{R}-\mu_{S}\right)}{k T}\right)}{\sum_{j=0}^{N} M_{j} \times \phi_{j} \times \exp \left(\frac{j \times\left(\mu_{R}-\mu_{S}\right)}{k T}\right)}
\end{aligned}
$$

which is independent of the value of $\mathrm{C}$.

Permutations with $\left[\mathrm{R}_{\mathrm{n}} \mathrm{S}_{\mathrm{N}-\mathrm{n}}\right]$ contain $\mathrm{n}$ subunits of $\mathrm{R}$ and $\mathrm{N}-\mathrm{n}$ subunits of $\mathrm{S}$. Thus, the mole fraction of $\mathrm{R}, X_{R}$, is given by

$$
\begin{aligned}
X_{R}= & \frac{[R]_{\text {total }}}{[R]_{\text {total }}+[S]_{\text {total }}}=\frac{\sum_{n=0}^{N} n \times\left[R_{n} S_{N-n}\right]}{\sum_{n=0}^{N} N \times\left[R_{n} S_{N-n}\right]} \\
= & \frac{\sum_{n=0}^{N} n \times M_{n} \times \phi_{n} \times r^{n} \times s^{N-n}}{\sum_{n=0}^{N} N \times M_{n} \times \phi_{n} \times r^{n} \times s^{N-n}}=\frac{\sum_{n=0}^{N} n \times M_{n} \times \phi_{n} \times\left(\frac{r}{s}\right)^{n}}{\sum_{n=0}^{N} N \times M_{n} \times \phi_{n} \times\left(\frac{r}{s}\right)^{n}}
\end{aligned}
$$

All mole fractions depend only on the ratio $r / s$ and $\varphi_{\mathrm{n}}$, and $X_{R}$ is a strictly monotonic function of $r / s$. Thus, if the mole fraction, $X_{R}$, and $\varphi_{\mathrm{n}}$ are known, eq 3 uniquely determines $r / s$. Knowing $r / s$, the value of any mole fraction, $X_{\mathrm{n}}$, can be computed using eq 2 .

In the special "statistical" case there is a simple analytic form for $r / s$. This case is examined in Section 63 . For the general case, $r / s$ is most easily determined numerically. A parametric approach is described in Sections 64 and 65. 
63. The statistical case. Eq 3 can be considerably simplified if every permutation (RRSRRS, SRRRRR, etc.) has the same free energy. In this case, $\phi_{\mathrm{n}}$ is independent of $\mathrm{n}$ so eq 3 simplifies to

$$
\begin{aligned}
X_{R}= & \frac{\sum_{n=0}^{N} n M_{n} \phi_{n} r^{n} s^{N-n}}{\sum_{n=0}^{N} N M_{n} \phi_{n} r^{n} s^{N-n}}=\frac{\sum_{n=0}^{N} n M_{n} r^{n} s^{N-n}}{\sum_{n=0}^{N} N M_{n} r^{n} s^{N-n}} \\
= & \frac{\sum_{n=0}^{N} n \times \frac{N !}{n !(N-n) !} \times r^{n} \times s^{N-n}}{\sum_{n=0}^{N} N \times \frac{N !}{n !(N-n) !} \times r^{n} \times s^{N-n}}
\end{aligned}
$$

Using the binomial expansion

$$
\sum_{j=0}^{N} \frac{N !}{j !(N-j) !} a^{j} b^{N-j}=(a+b)^{N}
$$

gives

$$
X_{R}=\frac{N \times r \times(r+s)^{N-1}}{N \times(r+s)^{N}}=\frac{r}{r+s} \quad \leftrightarrow \quad \frac{r}{s}=\frac{X_{R}}{1-X_{R}}
$$

which is an explicit expression for $r / s$ as a function of $X_{R}$. Substituting eq 4 into eq 2 determines the concentration of any species in solution in terms of $X_{R}$. The mole fraction of $\left[\mathrm{R}_{\mathrm{n}} \mathrm{S}_{\mathrm{N}-\mathrm{n}}\right], X_{\mathrm{n}}$, is equal to

$$
\begin{aligned}
X_{n} & =\frac{M_{n} \times \phi_{n} \times r^{n} \times s^{N-n}}{\sum_{j=0}^{N} M_{j} \times \phi_{j} \times r^{j} s^{N-j}}=\frac{\frac{N !}{n !(N-n) !} \times r^{n} \times s^{N-n}}{\sum_{j=0}^{N} \frac{N !}{j !(N-j) !} \times r^{j} \times s^{N-j}}=\frac{N !}{n !(N-n) !} \times \frac{r^{n} \times s^{N-n}}{(r+s)^{N}} \\
& =\frac{N !}{n !(N-n) !} \times X_{R}^{n} \times\left(1-X_{R}\right)^{N-n}
\end{aligned}
$$


The experiment measures combinations of $X_{\mathrm{n}}+X_{6-\mathrm{n}}$. For the specific case of $\mathrm{N}=6$,

$$
\begin{aligned}
& \text { Mole fraction of }\left[\mathrm{R}_{0} \mathrm{~S}_{6}\right]+\left[\mathrm{R}_{6} \mathrm{~S}_{0}\right]=X_{0}+X_{6} \\
& \text { Mole fraction of }\left[\mathrm{R}_{1} \mathrm{~S}_{5}\right]+\left[\mathrm{R}_{5} \mathrm{~S}_{1}\right]=X_{1}+X_{5} \\
& \text { Mole fraction of }\left[\mathrm{R}_{2} \mathrm{~S}_{4}\right]+\left[\mathrm{R}_{4} \mathrm{~S}_{2}\right]=X_{2}+X_{4} \\
& \text { Mole fraction of }\left[\mathrm{R}_{3} \mathrm{~S}_{3}\right]=X_{3}
\end{aligned}
$$

Using eq 5 these are equal to,

$$
\begin{aligned}
& \text { Mole fraction of }\left[\mathrm{R}_{0} \mathrm{~S}_{6}\right]+\left[\mathrm{R}_{6} \mathrm{~S}_{0}\right]=X_{\mathrm{R}}^{6}+\left(1-X_{\mathrm{R}}\right)^{6} \\
& \text { Mole fraction of }\left[\mathrm{R}_{1} \mathrm{~S}_{5}\right]+\left[\mathrm{R}_{5} \mathrm{~S}_{1}\right]=6 X_{\mathrm{R}}^{5}\left(1-X_{\mathrm{R}}\right)+6 X_{\mathrm{R}}\left(1-X_{\mathrm{R}}\right)^{5} \\
& \text { Mole fraction of }\left[\mathrm{R}_{2} \mathrm{~S}_{4}\right]+\left[\mathrm{R}_{4} \mathrm{~S}_{2}\right]=15 X_{\mathrm{R}}^{4}\left(1-X_{\mathrm{R}}\right)^{2}+15 X_{\mathrm{R}}^{2}\left(1-X_{\mathrm{R}}\right)^{4} \\
& \text { Mole fraction of }\left[\mathrm{R}_{3} \mathrm{~S}_{3}\right]=20 X_{\mathrm{R}}^{3}\left(1-X_{\mathrm{R}}\right)^{3}
\end{aligned}
$$

The above formulae are used to plot all four populations as a function of $X_{R}$. Because there are no free parameters, the experimental data either matches this plot, or the assumption that $\varphi_{\mathrm{n}}$ does not depend on $\mathrm{n}$ is wrong.

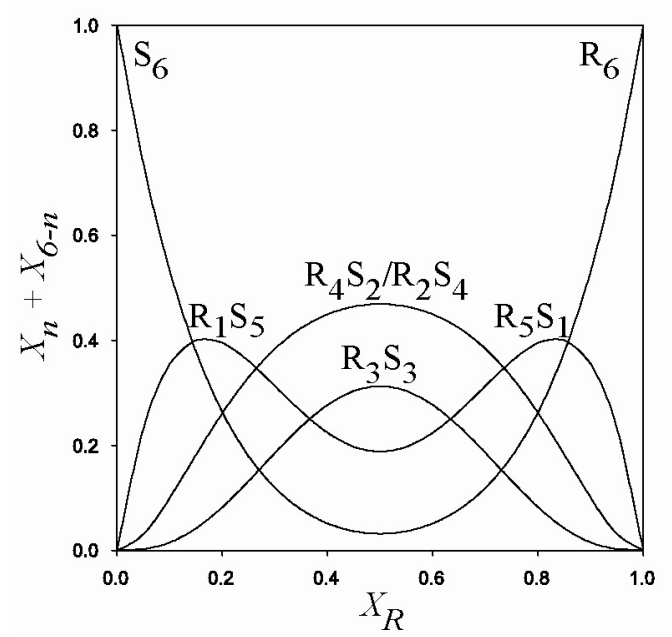


64. The parametric method. In general, each permutation can differ in stability, so $\varphi_{\mathrm{n}}$ depends on $\mathrm{n}$. In this case, there is not a simple analytic expression for $X_{\mathrm{n}}$ as a function of $X_{R}$ and $\varphi_{\mathrm{n}}$. However, eqs 2 and 3 permit one to evaluate $X_{R}$ and $X_{\mathrm{n}}$ as functions of $r / s$. For example, when N $=6$, the total mole fraction of $\mathrm{R}$ is

$$
X_{R}=\frac{\sum_{n=0}^{N} n M_{n} \phi_{n} r^{n} s^{N-n}}{\sum_{n=0}^{N} N M_{n} \phi_{n} r^{n} s^{N-n}}=\frac{\phi_{1} r^{1} s^{5}+5 \phi_{2} r^{2} s^{4}+10 \phi_{3} r^{3} s^{3}+10 \phi_{4} r^{4} s^{2}+5 \phi_{5} r^{5} s^{1}+\phi_{6} r^{6}}{\phi_{0} s^{6}+6 \phi_{1} r^{1} s^{5}+15 \phi_{2} r^{2} s^{4}+20 \phi_{3} r^{3} s^{3}+15 \phi_{4} r^{4} s^{2}+6 \phi_{5} r^{5} s^{1}+\phi_{6} r^{6}}
$$

and the experimentally measured mole fractions, $X_{\mathrm{n}}$, are

$$
\begin{aligned}
X_{0}+X_{6} & =\frac{\phi_{0} s^{6}+\phi_{6} r^{6}}{\phi_{0} s^{6}+6 \phi_{1} r^{1} s^{5}+15 \phi_{2} r^{2} s^{4}+20 \phi_{3} r^{3} s^{3}+15 \phi_{4} r^{4} s^{2}+6 \phi_{5} r^{5} s^{1}+\phi_{6} r^{6}} \\
X_{1}+X_{5}= & \frac{6\left(\phi_{1} r^{1} s^{5}+\phi_{5} r^{5} s^{1}\right)}{\phi_{0} s^{6}+6 \phi_{1} r^{1} s^{5}+15 \phi_{2} r^{2} s^{4}+20 \phi_{3} r^{3} s^{3}+15 \phi_{4} r^{4} s^{2}+6 \phi_{5} r^{5} s^{1}+\phi_{6} r^{6}} \\
X_{2}+X_{4}= & \frac{15\left(\phi_{2} r^{2} s^{4}+\phi_{4} r^{4} s^{2}\right)}{\phi_{0} s^{6}+6 \phi_{1} r^{1} s^{5}+15 \phi_{2} r^{2} s^{4}+20 \phi_{3} r^{3} s^{3}+15 \phi_{4} r^{4} s^{2}+6 \phi_{5} r^{5} s^{1}+\phi_{6} r^{6}} \\
X_{3}= & \frac{20 \phi_{3} r^{3} s^{3}}{\phi_{0} s^{6}+6 \phi_{1} r^{1} s^{5}+15 \phi_{2} r^{2} s^{4}+20 \phi_{3} r^{3} s^{3}+15 \phi_{4} r^{4} s^{2}+6 \phi_{5} r^{5} s^{1}+\phi_{6} r^{6}}
\end{aligned}
$$

For a given value of $X_{R}$ and $\varphi_{\mathrm{n}}$, one may determine the required value of $r / s$ via numeric inversion of eq 6 or by graphing $X_{R}$ versus $r$. Using the obtained value $r / s$ and eqs 7-10, one can then compute the populations. A graphical depiction of the parametric approach is described below for the case where $\varphi_{0}=\varphi_{6}=1, \varphi_{1}=\varphi_{5}=1.5, \varphi_{2}=\varphi_{4}=5, \varphi_{3}=10$.

Since the above equations only depend on the ratio, $r / s$, for convenience we may define

$$
s=1-r \leftrightarrow \frac{r}{s}=\frac{r}{1-r}
$$


Using eq 6, we obtain the following plot for the example where $\varphi_{0}=\varphi_{6}=1, \varphi_{1}=\varphi_{5}=1.5, \varphi_{2}=\varphi_{4}$ $=5, \varphi_{3}=10$.

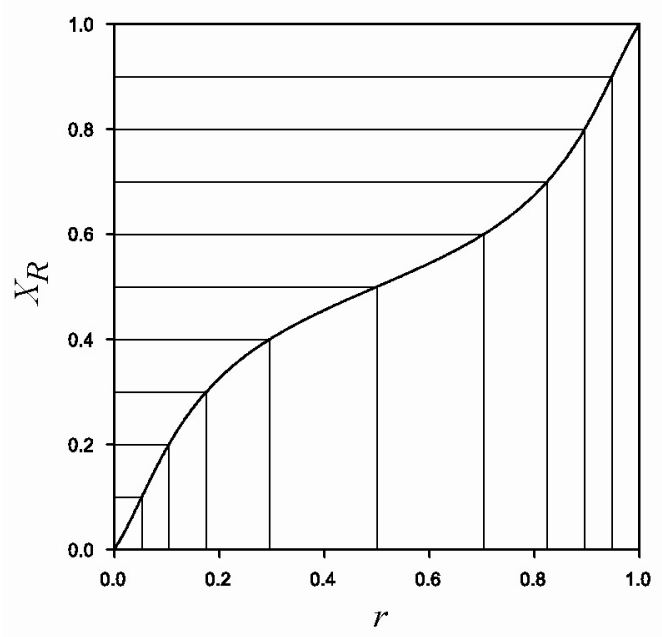

The drop lines depicted for each $X_{R}$ allow one to determine the corresponding value of $r$. For this example we obtain the following values of $r$ at each $X_{R}$.

\begin{tabular}{ccc}
\hline$X_{R}$ & $r$ & $r / s$ \\
\hline 0 & 0 & 0 \\
0.1 & 0.0522 & 0.0551 \\
0.2 & 0.104 & 0.116 \\
0.3 & 0.175 & 0.213 \\
0.4 & 0.296 & 0.421 \\
0.5 & 0.50 & 1.0 \\
0.6 & 0.704 & 2.377 \\
0.7 & 0.825 & 4.706 \\
0.8 & 0.896 & 8.643 \\
0.9 & 0.948 & 18.14 \\
1.0 & 1.0 & infinity \\
\hline & &
\end{tabular}

Using these values of $r / s$, we compute $X_{0}+X_{6}, X_{1}+X_{5}, X_{2}+X_{4}$, and $X_{3}$ using eqs 7-10. The results are plotted below. 


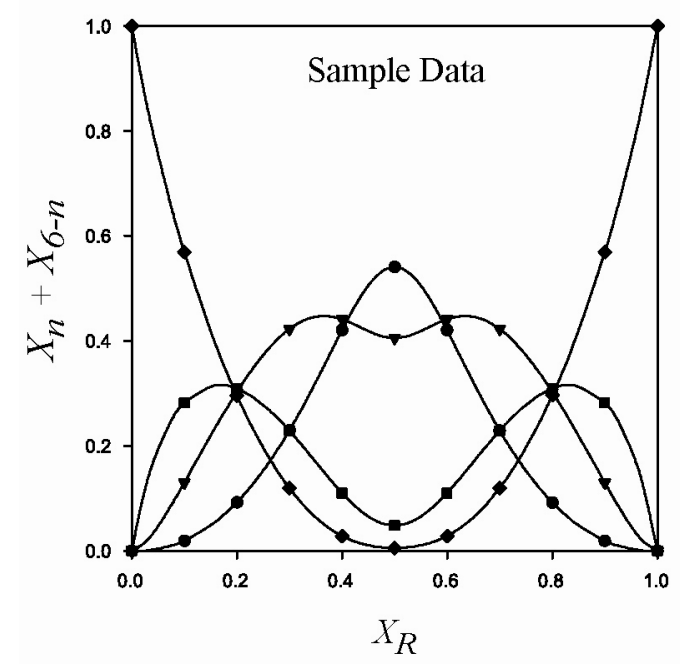

$\mathbf{R}_{3} \mathbf{S}_{3}(\bullet) ; \mathbf{R}_{4} \mathbf{S}_{2} / \mathbf{R}_{2} \mathbf{S}_{4}(\boldsymbol{\nabla}) ; \mathbf{R}_{5} \mathbf{S}_{\mathbf{1}} / \mathbf{R}_{\mathbf{1}} \mathbf{S}_{\mathbf{5}}(\boldsymbol{\bullet}) ; \mathbf{R}_{6} / \mathbf{S}_{\mathbf{6}}(\bullet)$

Comparing the above plot with the plot obtained in the statistical case (Section 63), there are several notable changes. For instance, the $\mathbf{R}_{\mathbf{4}} \mathbf{S}_{2} / \mathbf{R}_{\mathbf{2}} \mathbf{S}_{4}$ curve now exhibits two maxima. Also, at $X_{R}$ $=0.5$, the dominant species is now $\mathbf{R}_{\mathbf{3}} \mathbf{S}_{3}$. These results match our expectations because $\varphi_{3}$ was set to be larger than all other $\varphi_{\mathrm{n}}$ 's $\left(\varphi_{0}=\varphi_{6}=1, \varphi_{1}=\varphi_{5}=1.5, \varphi_{2}=\varphi_{4}=5, \varphi_{3}=10\right)$. 
65. Fitting the experimental data with the parametric method. To compare the theory directly to experiment, one can refine an initial guess of $\varphi_{n}$ until the predicted populations for the experimental values of $X_{R}$ best fit the experimental populations. An adaptive step algorithm iteratively adjusts $\varphi_{\mathrm{n}}$ to minimize the root mean square error in the predicted populations. N/2 of the $\varphi_{\mathrm{n}}$ variables are independent, and for $\mathrm{N}=6, \varphi_{1}, \varphi_{2}$, and $\varphi_{3}$ are a convenient choice. A software package that supports nonlinear least-squares fitting of parametric equations is required. The root mean square deviation between the hexamer model and the data is $2.9 \%$.

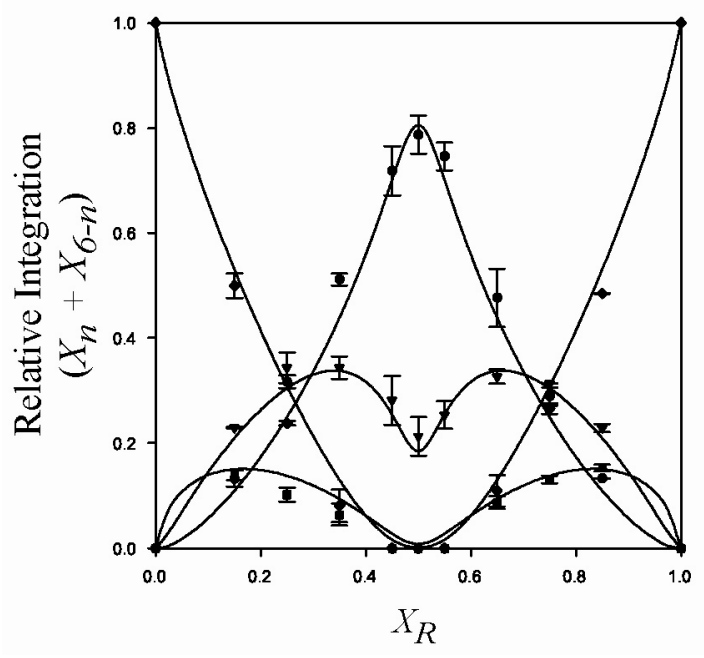

$(\bullet) \mathbf{R}_{3} \mathbf{S}_{3} ;(\boldsymbol{v}) \mathbf{R}_{4} \mathbf{S}_{2} / \mathbf{R}_{2} \mathbf{S}_{4} ;(\bullet) \mathbf{R}_{5} \mathbf{S}_{1} / \mathbf{R}_{1} \mathbf{S}_{5} ;(\bullet) \mathbf{R}_{6} / \mathbf{S}_{6}$ •

Best fit values of $\varphi$

\begin{tabular}{ccccccc}
\hline$\varphi_{0}$ & $\varphi_{1}$ & $\varphi_{2}$ & $\varphi_{3}$ & $\varphi_{4}$ & $\varphi_{5}$ & $\varphi_{6}$ \\
\hline 1.0 & 0.79 & 7.3 & 47.7 & 7.3 & 0.79 & 1.0 \\
\hline
\end{tabular}

Percent Errors in $\varphi_{n}$

\begin{tabular}{ccccc}
\hline $\mathrm{n}$ & 0 & 1 & 2 & 3 \\
\hline$\varphi_{\mathrm{n}} / \varphi_{\mathrm{o}}$ & 0 & 3.7 & 6.5 & 9.4 \\
$\varphi_{\mathrm{n}} / \varphi_{1}$ & 3.7 & 0 & 3.7 & 6.5 \\
$\varphi_{\mathrm{n}} / \varphi_{2}$ & 6.5 & 3.7 & 0 & 2.8 \\
$\varphi_{\mathrm{n}} / \varphi_{3}$ & 9.4 & 6.5 & 2.8 & 0 \\
\hline
\end{tabular}


66. Equilibrium constants. The "free energy coefficients" of $R_{n} S_{N-n}\left(\varphi_{n}\right)$ are related to the equilibrium constants as follows

$$
\begin{aligned}
& \mathrm{R}_{3} \mathrm{~S}_{3} \stackrel{K_{1}}{\rightleftharpoons} 1 / 2 \mathrm{R}_{6}+1 / 2 \mathrm{~S}_{6} \\
& \mathrm{R}_{3} \mathrm{~S}_{3} \stackrel{K_{2}}{\rightleftharpoons} 1 / 2 \mathrm{R}_{5} \mathrm{~S}_{1}+1 / 2 \mathrm{R}_{1} \mathrm{~S}_{5} \\
& \mathrm{R}_{3} \mathrm{~S}_{3} \stackrel{K_{3}}{\rightleftharpoons} 1 / 2 \mathrm{R}_{4} \mathrm{~S}_{2}+1 / 2 \mathrm{R}_{2} \mathrm{~S}_{4}
\end{aligned}
$$

We now express $K_{1}, K_{2}$ and $K_{3}$ in terms of $\varphi_{\mathrm{n}}$ using eq 1 .

$$
K_{1}=\frac{\left[\mathrm{R}_{6}\right]^{1 / 2} \times\left[\mathrm{S}_{6}\right]^{1 / 2}}{\left[\mathrm{R}_{3} \mathrm{~S}_{3}\right]}
$$

Substituting the concentrations into eq 1

$$
\left[S_{6}\right]=C \times M_{0} \times \phi_{0} \times s^{6} \quad\left[R_{6}\right]=C \times M_{6} \times \phi_{6} \times r^{6} \quad\left[R_{3} S_{3}\right]=C \times M_{3} \times \phi_{3} \times r^{3} \times s^{3}
$$

which then give,

$$
K_{1}=\frac{\left(C M_{0} \phi_{0} s^{6}\right)^{1 / 2} \times\left(C M_{6} \phi_{6} r^{6}\right)^{1 / 2}}{\left(C M_{3} \phi_{3} r^{3} s^{3}\right)}=\frac{\phi_{0}}{20 \phi_{3}}
$$

For the above reactions,

$$
K_{1}=\frac{\phi_{0}}{20 \phi_{3}} \quad K_{2}=\frac{3 \phi_{1}}{10 \phi_{3}} \quad K_{3}=\frac{3 \phi_{2}}{4 \phi_{3}}
$$

For the general case,

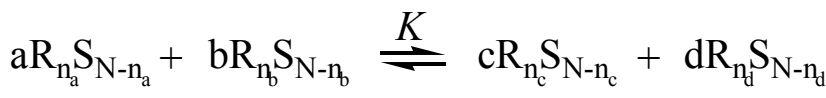

$$
\begin{aligned}
& K=\frac{\left(M_{n_{c}} \phi_{n_{c}}\right)^{c} \times\left(M_{n_{d}} \phi_{n_{d}}\right)^{d}}{\left(M_{n_{a}} \phi_{n_{a}}\right)^{a} \times\left(M_{n_{b}} \phi_{n_{b}}\right)^{b}}
\end{aligned}
$$




\begin{tabular}{ccccc}
\hline $\mathrm{m}$ & $K_{\text {statistical }}$ & $\Delta G_{\text {statistical }}(\mathrm{kcal} / \mathrm{mol})^{a}$ & $K_{\text {experimental }}$ & $\Delta G_{\text {non-statistical }}(\mathrm{kcal} / \mathrm{mol})^{b}$ \\
\hline 1 & 0.05 & 1.33 & $1.0 \pm 0.1 \times 10^{-3}$ & $1.73 \pm 0.04$ \\
2 & 0.30 & 0.53 & $5.0 \pm 0.3 \times 10^{-3}$ & $1.82 \pm 0.03$ \\
3 & 0.75 & 0.13 & $115 \pm 3 \times 10^{-3}$ & $0.83 \pm 0.01$ \\
\hline
\end{tabular}

${ }^{a} \Delta G_{\text {statistical }}=-R T \ln K_{\text {statistical }}$

${ }^{b} \Delta G_{\text {non-statistical }}=-R T \ln \left(K_{\text {experimental }} / K_{\text {statistical }}\right)$ 
67. Heptamer fit to data. If the subunits assemble into heptamers, the concentration of $\left[\mathrm{R}_{n} \mathrm{~S}_{7-\mathrm{n}}\right]$ can be written using eq 1 .

$$
\left[R_{n} S_{7-n}\right]=C \times \frac{7 !}{(7-n) ! \times n !} \times \phi_{n} \times r^{n} \times s^{7-n}
$$

Similarly, from eqs 2 and 3, the mole fraction of subunits of $\mathrm{R}, X_{\mathrm{R}}$, is given by

$$
\begin{aligned}
X_{R} & =\frac{\sum_{n=0}^{N} n \times M_{n} \times \phi_{n} \times r^{n} \times s^{N-n}}{\sum_{n=0}^{N} N \times M_{n} \times \phi_{n} \times r^{n} \times s^{N-n}} \\
& =\frac{\phi_{1} r^{1} s^{6}+6 \phi_{2} r^{2} s^{5}+15 \phi_{3} r^{3} s^{4}+20 \phi_{4} r^{4} s^{3}+15 \phi_{5} r^{5} s^{2}+6 \phi_{6} r^{6} s^{1}+\phi_{7} r^{7}}{\phi_{0} r^{0} s^{7}+7 \phi_{1} r^{1} s^{6}+21 \phi_{2} r^{2} s^{5}+35 \phi_{3} r^{3} s^{4}+35 \phi_{4} r^{4} s^{3}+21 \phi_{5} r^{5} s^{2}+7 \phi_{6} r^{6} s^{1}+\phi_{7} r^{7}}
\end{aligned}
$$

and the experimentally measured mole fractions, $X_{\mathrm{n}}$, are

$$
\begin{aligned}
& X_{0}+X_{7}=\frac{\phi_{0} s^{7}+\phi_{7} r^{7}}{\phi_{0} s^{7}+7 \phi_{1} r^{1} s^{6}+21 \phi_{2} r^{2} s^{5}+35 \phi_{3} r^{3} s^{4}+35 \phi_{4} r^{4} s^{3}+21 \phi_{5} r^{5} s^{2}+7 \phi_{6} r^{6} s^{1}+\phi_{7} r^{7}} \\
& X_{1}+X_{6}=\frac{7\left(\phi_{1} r^{1} s^{6}+\phi_{6} r^{6} s^{1}\right)}{\phi_{0} s^{7}+7 \phi_{1} r^{1} s^{6}+21 \phi_{2} r^{2} s^{5}+35 \phi_{3} r^{3} s^{4}+35 \phi_{4} r^{4} s^{3}+21 \phi_{5} r^{5} s^{2}+7 \phi_{6} r^{6} s^{1}+\phi_{7} r^{7}} \\
& X_{2}+X_{5}=\frac{21\left(\phi_{2} r^{2} s^{5}+\phi_{5} r^{5} s^{2}\right)}{\phi_{0} s^{7}+7 \phi_{1} r^{1} s^{6}+21 \phi_{2} r^{2} s^{5}+35 \phi_{3} r^{3} s^{4}+35 \phi_{4} r^{4} s^{3}+21 \phi_{5} r^{5} s^{2}+7 \phi_{6} r^{6} s^{1}+\phi_{7} r^{7}} \\
& X_{3}+X_{4}=\frac{35\left(\phi_{3} r^{3} s^{4}+\phi_{4} r^{4} s^{3}\right)}{\phi_{0} s^{7}+7 \phi_{1} r^{1} s^{6}+21 \phi_{2} r^{2} s^{5}+35 \phi_{3} r^{3} s^{4}+35 \phi_{4} r^{4} s^{3}+21 \phi_{5} r^{5} s^{2}+7 \phi_{6} r^{6} s^{1}+\phi_{7} r^{7}}
\end{aligned}
$$

The figure shows the results of a parametric non-linear least-squares fit of the heptamer model to the experimental data. The root mean square deviation between the heptamer model and data is $1.6 \%$. 


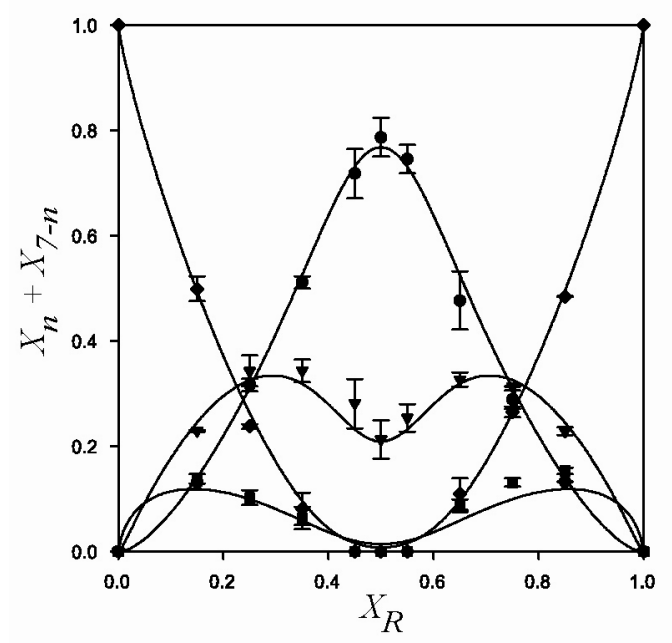

$\mathbf{R}_{4} \mathbf{S}_{3} / \mathbf{R}_{3} \mathbf{S}_{4}(\bullet) ; \mathbf{R}_{2} \mathbf{S}_{5} / \mathbf{R}_{5} \mathbf{S}_{2}(\boldsymbol{v}) ; \mathbf{R}_{\mathbf{1}} \mathbf{S}_{\mathbf{6}} / \mathbf{R}_{6} \mathbf{S}_{\mathbf{1}}(\bullet) ; \mathbf{R}_{7} / \mathbf{S}_{7}(\bullet)$.

Best fit values of $\varphi$

\begin{tabular}{cccccccc}
\hline$\varphi_{0}$ & $\varphi_{1}$ & $\varphi_{2}$ & $\varphi_{3}$ & $\varphi_{4}$ & $\varphi_{5}$ & $\varphi_{6}$ & $\varphi_{7}$ \\
\hline 1.0 & 0.31 & 1.39 & 2.93 & 2.93 & 1.39 & 0.31 & 1.0 \\
\hline
\end{tabular}

Percent Errors in $\varphi_{n}$

\begin{tabular}{ccccc}
\hline $\mathrm{n}$ & 0 & 1 & 2 & 3 \\
\hline$\varphi_{\mathrm{n}} / \varphi_{\mathrm{o}}$ & 0 & 4.3 & 6.7 & 7.2 \\
$\varphi_{\mathrm{n}} / \varphi_{1}$ & 4.3 & 0 & 2.2 & 5.1 \\
$\varphi_{\mathrm{n}} / \varphi_{2}$ & 6.7 & 2.2 & 0 & 2.4 \\
$\varphi_{\mathrm{n}} / \varphi_{3}$ & 7.2 & 5.1 & 2.4 & 0 \\
\hline
\end{tabular}


68. Generic tetramer Job plot. For the case where subunits A and B assemble into tetramers, the mole fraction of $\mathrm{A}, X_{\mathrm{A}}$, is related to the chemical potential using eqs 2-3.

$$
X_{A}=\frac{\sum_{n=0}^{N} n \times M_{n} \times \phi_{n} \times a^{n} \times b^{N-n}}{\sum_{n=0}^{N} N \times M_{n} \times \phi_{n} \times a^{n} \times b^{N-n}}=\frac{\phi_{1} a^{1} b^{3}+3 \phi_{2} a^{2} b^{2}+3 \phi_{3} a^{3} b^{1}+\phi_{4} a^{4}}{\phi_{0} b^{4}+4 \phi_{1} a^{1} b^{3}+6 \phi_{2} a^{2} b^{2}+4 \phi_{3} a^{3} b^{1}+\phi_{4} a^{4}}
$$

While the populations, $X_{\mathrm{n}}$, are

$$
\begin{aligned}
& X_{0}=\frac{\phi_{0} b^{4}}{\phi_{0} b^{4}+4 \phi_{1} a^{1} b^{3}+6 \phi_{2} a^{2} b^{2}+4 \phi_{3} a^{3} b^{1}+\phi_{4} a^{4}} \\
& X_{1}=\frac{4 \phi_{1} a^{1} b^{3}}{\phi_{0} b^{4}+4 \phi_{1} a^{1} b^{3}+6 \phi_{2} a^{2} b^{2}+4 \phi_{3} a^{3} b^{1}+\phi_{4} a^{4}} \\
& X_{2}=\frac{6 \phi_{2} a^{2} b^{2}}{\phi_{0} b^{4}+4 \phi_{1} a^{1} b^{3}+6 \phi_{2} a^{2} b^{2}+4 \phi_{3} a^{3} b^{1}+\phi_{4} a^{4}} \\
& X_{3}=\frac{4 \phi_{3} a^{3} b^{1}}{\phi_{0} b^{4}+4 \phi_{1} a^{1} b^{3}+6 \phi_{2} a^{2} b^{2}+4 \phi_{3} a^{3} b^{1}+\phi_{4} a^{4}} \\
& X_{4}=\frac{\phi_{4} a^{4}}{\phi_{0} b^{4}+4 \phi_{1} a^{1} b^{3}+6 \phi_{2} a^{2} b^{2}+4 \phi_{3} a^{3} b^{1}+\phi_{4} a^{4}}
\end{aligned}
$$

The above formulae are used to plot all five populations as a function of $X_{\mathrm{A}}$ for a statistical distribution of species $\left(\varphi_{0}=\varphi_{1}=\ldots=\varphi_{4}\right)$.

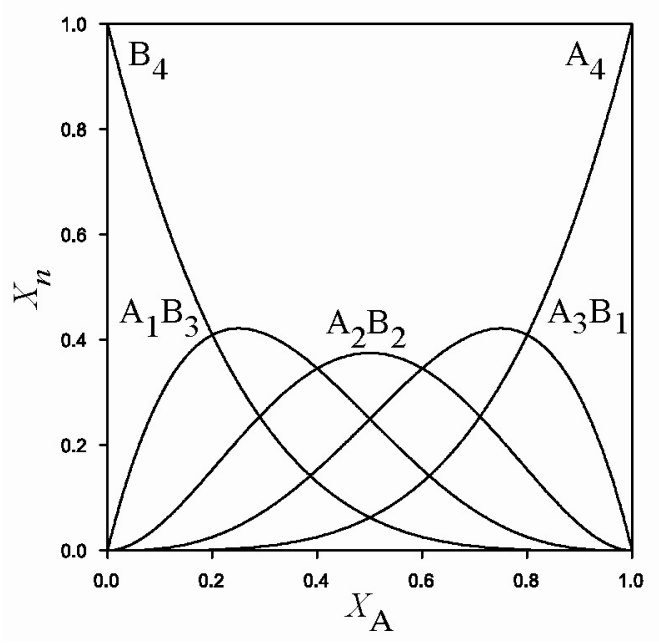


69. Dimer-tetramer Job plot. In this section we consider the case where two subunits, A and B, assemble to form species with different aggregation numbers (e.g., dimers and tetramers).

$\phi_{\mathrm{Nn}}(0 \leq \mathrm{n} \leq \mathrm{N})$ describes the mean free energy of $\mathrm{N}$-mer aggregates with $\mathrm{n}$ subunits of $\mathrm{A}$ and $\mathrm{N}-\mathrm{n}$ subunits of $\mathrm{B}$.

$$
\left[A_{n} B_{N-n}\right]=C \times M_{n} \times \phi_{N n} \times a^{n} \times b^{N-n}
$$

The concentration of $[\mathrm{A}]$ and $[\mathrm{B}]$ uniquely determine $a$ and $b$ by the implicit equations,

$$
\begin{aligned}
& {[A]=\sum_{N, n} n \times\left[A_{n} B_{N-n}\right]=\sum_{N, n} n \times C \times \frac{N !}{(N-n) ! \times n !} \times \phi_{N n} \times a^{n} \times b^{N-n}} \\
& {[B]=\sum_{N, n}(N-n) \times\left[A_{n} B_{N-n}\right]=\sum_{N, n}(N-n) \times C \times \frac{N !}{(N-n) ! \times n !} \times \phi_{N n} \times a^{n} \times b^{N-n}}
\end{aligned}
$$

The mole fraction of $\left[\mathrm{A}_{\mathrm{n}} \mathrm{B}_{\mathrm{N}-\mathrm{n}}\right], X_{\mathrm{N}, \mathrm{n}}$, is given by

$$
X_{N n}=\frac{N \times\left[A_{n} B_{N-n}\right]}{[A]+[B]}=\frac{N \times \frac{N !}{(N-n) ! \times n !} \times \phi_{N n} \times a^{n} b^{N-n}}{\sum_{J, j} J \times \frac{J !}{(J-j) ! \times j !} \times \phi_{J j} \times a^{j} b^{J-j}}
$$

For illustrative purposes, we consider the specific case of dimers and tetramers in equilibrium where species A forms dimers $\left(\mathrm{A}_{2}\right)$ and species $\mathrm{B}$ readily forms tetramers $\left(\mathrm{B}_{4}\right)$. Mixing $\mathrm{A}$ and $\mathrm{B}$ will give rise to a heterodimer $(A B)$ and several heterotetramers $\left(A_{3} B_{1}, A_{2} B_{2}, A_{1} B_{3}\right)$ in addition to the homodimer $\left(\mathrm{A}_{2}\right)$ and homotetramer $\left(\mathrm{B}_{4}\right)$.

The concentrations of $[\mathrm{A}]$ and $[\mathrm{B}]$ determine the values of $a$ and $b$ by the implicit equations,

$$
\begin{gathered}
{[A]=C \times\left(2 \phi_{21} a^{1} b^{1}+2 \phi_{22} a^{2}+4 \phi_{41} a^{1} b^{3}+12 \phi_{42} a^{2} b^{2}+12 \phi_{43} a^{3} b^{1}\right)} \\
{[B]=C \times\left(2 \phi_{21} a^{1} b^{1}+4 \phi_{40} b^{4}+12 \phi_{41} a^{1} b^{3}+12 \phi_{42} a^{2} b^{2}+4 \phi_{43} a^{3} b^{1}\right)}
\end{gathered}
$$

The dimer mole fractions are given by, 


$$
X_{A_{2}}=\frac{2 C \phi_{22} a^{2}}{[A]+[B]} \quad X_{A B}=\frac{4 C \phi_{21} a^{1} b^{1}}{[A]+[B]}
$$

and the tetramer mole fractions are given by,

$$
\begin{array}{ll}
X_{A_{3} B_{1}}=\frac{16 C \phi_{43} a^{3} b^{1}}{[A]+[B]} & X_{A_{2} B_{2}}=\frac{24 C \phi_{42} a^{2} b^{2}}{[A]+[B]} \\
X_{A_{1} B_{3}}=\frac{16 C \phi_{41} a^{1} b^{3}}{[A]+[B]} & X_{B_{4}}=\frac{4 C \phi_{40} b^{4}}{[A]+[B]}
\end{array}
$$

As one would anticipate, the relative aggregate populations depend on the mole fraction of $\mathrm{A}, X_{\mathrm{A}}$, and the total concentration of [A] and [B]. Qualitatively, increasing $X_{\mathrm{A}}$ will favor aggregates rich in A while increasing the overall concentration will favor the tetramers. Simultaneous fits of plots at several overall concentrations would provide more accurate free energies. For example, we have plotted representative plots for low (4), moderate (16) and high (100) total concentrations of $[\mathrm{A}]$ and $[\mathrm{B}]$ for a given set of $\phi_{\mathrm{Nn}}$.
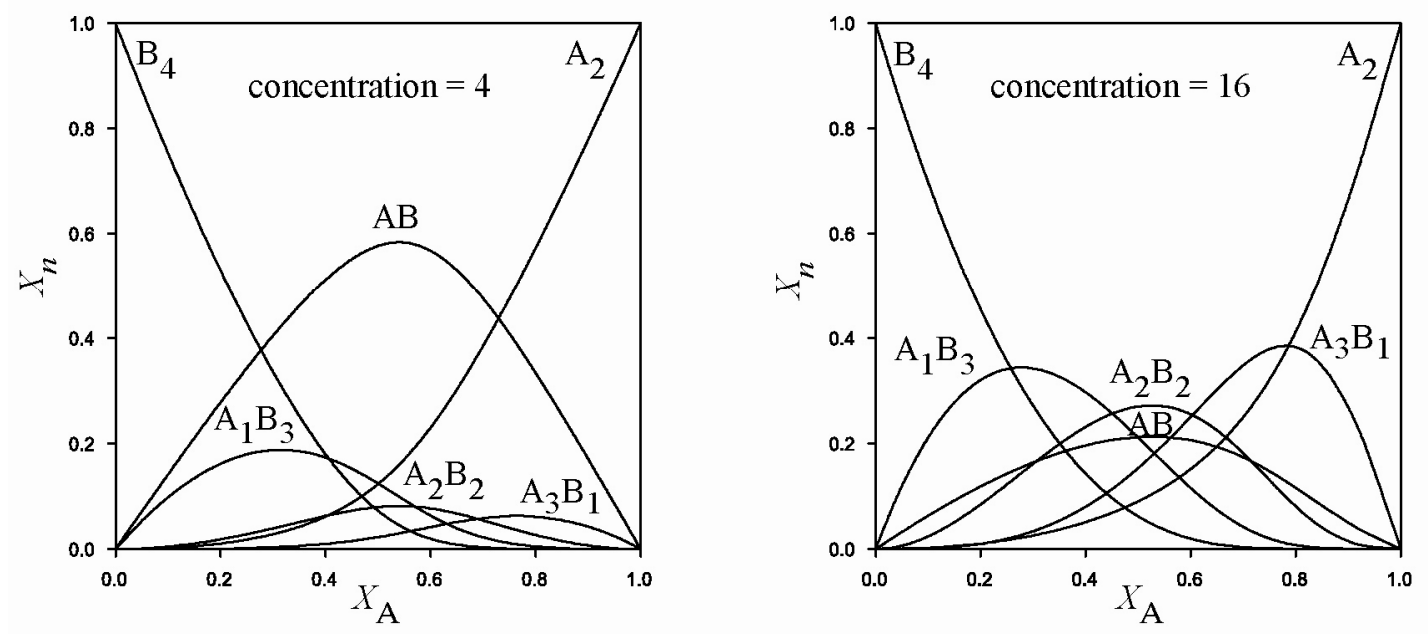


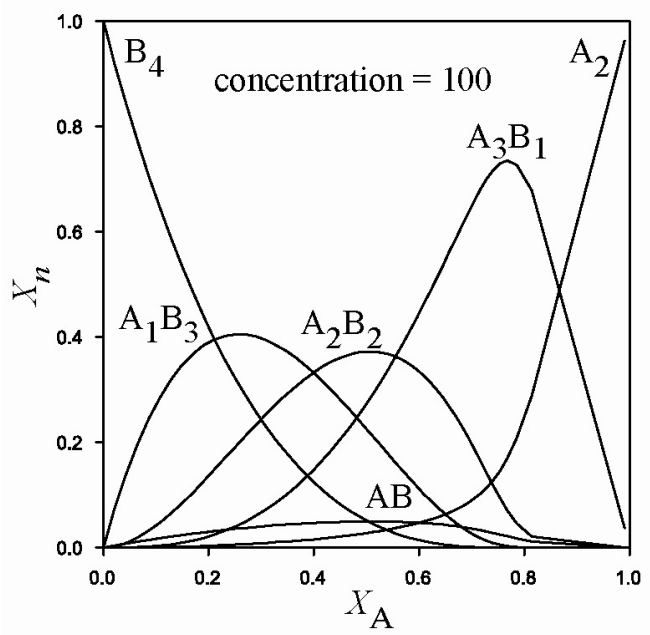

